\title{
Algebraic diagrammatic construction formalism with three-body interactions
}

\author{
Francesco Raimondi and Carlo Barbieri \\ Department of Physics, University of Surrey, Guildford GU2 7XH, United Kingdom
}

(Dated: October 10, 2018)

\begin{abstract}
Background: Self-consistent Green's function theory has recently been extended to the basic formalism needed to account for three-body interactions [A. Carbone, A. Cipollone, C. Barbieri, A. Rios, and A. Polls, Phys. Rev. C 88, 054326 (2013)]. The contribution of three-nucleon forces has so far been included in ab initio calculations on nuclear matter and finite nuclei only as averaged two-nucleon forces.

Purpose: We derive the working equations for all possible two- and three-nucleon terms that enter the expansion of the self-energy up to the third order, thus including the interaction-irreducible (i.e., not averaged) diagrams with three-nucleon forces that have been previously neglected.

Methods: We employ the algebraic diagrammatic construction up to the third order as an organization scheme for generating a non perturbative self-energy, in which ring (particle-hole) and ladder (particle-particle) diagrams are resummed to all orders.

Results: We derive expressions of the static and dynamic self-energy up to the third order, by taking into account the set of diagrams required when either the skeleton or nonskeleton expansions of the single-particle propagator are assumed. A hierarchy of importance among different diagrams is revealed, and a particular emphasis is given to a third-order diagram (see Fig. 2c) that is expected to play a significant role among those featuring an interaction-irreducible three-nucleon force.

Conclusion: A consistent formalism to resum at infinite order correlations induced by three-nucleon forces in the self-consistent Green's function theory is now available and ready to be implemented in the many-body solvers.
\end{abstract}

\section{INTRODUCTION}

Three-body interactions play a prominent role in determining the behaviour of strongly interacting quantum systems [1]. For instance, three-nucleon forces (3NFs) are necessary to reproduce the saturation of infinite matter as well as to determine the structure and location of the driplines in neutron rich isotopes. Hence, they have been implemented in most of the post-Hartree-Fock approaches that are currently used to study medium mass isotopes, such as self-consistent Green's function (SCGF) theory [2, 3], the coupled cluster method [4, 5], and the in-medium similarity renormalization group [6, 7]. In all of these methods, one typically proceeds by performing a normal ordering of the Hamiltonian, or a similar averaging, so that the dominant effect of $3 \mathrm{NF}$ s can be taken into account as an effective nucleon-nucleon force (2NF). Advances of the above many-body methods, with the concurrent introduction of chiral two- and three-nucleon interactions, based on the symmetries of QCD [8, 9], have led recently to remarkable successes in nuclear $a b$ initio theory [10 12. Presently, the major sources of error in first-principle predictions originate from uncertainties of the nuclear Hamiltonian [10, 13. However, the expected progress in the next-generation realistic interactions will eventually require further developments of the many-body formalisms.

The SCGF theory is a quantum many-body method that has been extensively applied to both condensed matter and nuclear systems [2, 14, 19]. This approach relies on the solution of the Dyson equation, which is an exact restatement of the many-body Schrödinger equation and it allows for a diagrammatic expansion with respect to the nuclear interaction. However, for nuclear structure and reaction studies, a perturbative expansion is not sufficient due to the strong nature of the nuclear force and the importance of the long-range correlations, which affect the propagation of nucleons inside the medium. In practice, one must resort to an efficient method in which entire classes of correlations are resummed non perturbatively.

For this purpose, a major challenge is to find a scheme capable of organizing the rapidly increasing number of Feynman diagrams entering the computation of Green's functions, especially when 3NFs and many-nucleon interactions are present. Ideally, one should include different classes of Feynman diagrams at all orders, i.e. in a nonperturbative way; at the same time, one needs to keep under control the computational resources required by the many-body problem, even for those postHartree-Fock approaches scaling polynomially with the number of nucleons. A powerful tool complying with these requirements has been devised 30 years ago in the Green's function theory applied to quantum chemistry. It is referred to as the algebraic diagrammatic construction (ADC) method [20, 21]. Born as a way to include third-order self-energy diagrams that are necessary to reproduce affinities and ionisation energies, the ADC also allows the infinite resummation of specific classes of diagrams, such as the ladder and ring series. The general procedure is to impose the correct spectral representation of the self-energy and to require that its perturbative expansion is also consistent with the Feynman diagram series up to a given order $n$. The spectral representation implies that diagrams up to order $n$ are actually taken as "seeds" for all-order resummations. This generates a hierarchy of many-body truncations, labelled as $\operatorname{ADC}(n)$, that contains selected relevant terms, it is non perturba- 
tive and can be systematically improved.

The aim of this paper is to derive the entire set of working equations for the $\mathrm{ADC}(3)$ self-energy, when $3 \mathrm{NFs}$ are present. The general formalism and diagrammatic rules for the SCGF theory with three-body interactions has been developed in Ref. 22. There, it was shown that the number of Feynman diagrams to be calculated can be reduced by introducing averaged effective interactions (similarly to the normal ordering strategy mentioned above), so that one needs to consider only interactionirreducible diagrams. Using the resulting effective $2 \mathrm{NFs}$, a set of applications of the SCGF was put forward with computations of binding energies 3, 23, spectral distributions and radii [12, 24] for closed subshell isotopes of medium-mass. For the nuclear matter case, saturation properties of chiral forces and other thermodynamical aspects have also been studied 25. Moreover, current efforts are devoted to describing the one-nucleon scattering on a nucleus within the same framework [26].

While Ref. 22 introduced the set of self-energy diagrams up to third order, the necessary formalism for extending them to a nonperturbative approach has not yet been derived. We fill this gap here by deriving explicit expressions of the $\mathrm{ADC}(3)$ based on the Feynman diagrams derived thereof. For this purpose we revisit the SCGF formalism in Sec. II] with special emphasis on how $3 \mathrm{NFs}$ are incorporated in the self-energy expansion. Section [II] discusses the ADC method at order $n$, i.e. $\operatorname{ADC}(n)$, and we derive the working equations at second and third order, $\operatorname{ADC}(2)$ and $\operatorname{ADC}(3)$, in full detail. In our derivation, a hierarchy of importance among different self-energy diagrams emerges naturally: it is based on intermediate excitation energies embedded in each diagram and on the relative importance between $2 \mathrm{~N}$ and $3 \mathrm{NFs}$. For the $\mathrm{ADC}(3)$, we present in Sec. III diagrams that contain only two-particle-one-hole $(2 p 1 h)$ and two-hole-oneparticle $(2 h 1 p)$ intermediate states with effective $2 \mathrm{NFs}$ and interaction-irreducible 3NFs. These diagrams are displayed in Fig. (2) below and are the dominant contributions to $\mathrm{ADC}(3)$. We also present additional equations for a subset of diagrams with $3 p 2 h$ and $3 h 2 p$ intermediate states, chosen from the different topologies appearing at third order. This will give a general overview of the formalism up to $n=3$. All the remaining parts of the $\mathrm{ADC}(3)$ equations, which complete the diagrams with $3 p 2 h$ and $3 h 2 p$ configurations, are given in Appendix A. In Appendix B we display the angular momentum coupled form for the leading contributions of Fig. (2). The diagram in Fig. 2c has not yet been included in calculations, but it could be added to current numerical implementations and it is expected to be the most important among those with an irreducible 3NF. In Appendix C we derive additional nonskeleton Feynman diagrams for both the static and dynamic self-energy that need to be included in non self-consistent calculations. The entire set of equations derived up to $\mathrm{ADC}(3)$ informs our conclusions, which are drawn in Sec. IV]

\section{SCGF FORMALISM WITH 3NFS}

Many-particle Green's functions, also known in the literature as propagators or correlation functions, are at the heart of the SCGF formalism. The simplest Green's function is the one-body (1B) propagator describing the in-medium propagation of a particle or an hole, which are respectively created and annihilated by field operators $a_{\beta}^{\dagger}$ and $a_{\alpha}$ in the quantum states $\beta$ and $\alpha$ [27, 28]:

$$
g_{\alpha \beta}\left(t-t^{\prime}\right)=-\frac{i}{\hbar}\left\langle\Psi_{0}^{A}\left|\mathcal{T}\left[a_{\alpha}(t) a_{\beta}^{\dagger}\left(t^{\prime}\right)\right]\right| \Psi_{0}^{A}\right\rangle .
$$

Here and in the following we use Greek indexes to label the states of a complete orthonormal single-particle basis $\{\alpha\}$, which could be discrete or having a continuous spectrum. The time interval $\left(t-t^{\prime}\right)$ of the propagation in Eq. (1) is ordered according to the action of the timeordering operator $\mathcal{T}$, which obeys the Fermi statistics. To describe the propagation of two particles and two holes, we introduce also the two-body (2B) Green's function,

$$
\begin{aligned}
& g_{\alpha \beta, \gamma \delta}^{I I}\left(t_{\alpha}, t_{\beta}, t_{\gamma}, t_{\delta}\right)= \\
& -\frac{i}{\hbar}\left\langle\Psi_{0}^{A}\left|\mathcal{T}\left[a_{\beta}\left(t_{\beta}\right) a_{\alpha}\left(t_{\alpha}\right) a_{\gamma}^{\dagger}\left(t_{\gamma}\right) a_{\delta}^{\dagger}\left(t_{\delta}\right)\right]\right| \Psi_{0}^{A}\right\rangle .
\end{aligned}
$$

For our purposes, we will concentrate on the propagator of Eq. (1), which is defined with respect to the exact $A$-body ground state $\left|\Psi_{0}^{A}\right\rangle$. The latter is the lowest eigenstate of the Schrödinger problem,

$$
\hat{H}\left|\Psi_{n}^{A}\right\rangle=E_{n}^{A}\left|\Psi_{n}^{A}\right\rangle .
$$

The Lehmann representation of the Green's function is obtained by Fourier transforming Eq. (1) to the energy plane. It contains the relevant informations on the singleparticle dynamics,

$$
\begin{aligned}
g_{\alpha \beta}(\omega) & =\sum_{n} \frac{\left\langle\Psi_{0}^{A}\left|a_{\alpha}\right| \Psi_{n}^{A+1}\right\rangle\left\langle\Psi_{n}^{A+1}\left|a_{\beta}^{\dagger}\right| \Psi_{0}^{A}\right\rangle}{\hbar \omega-\left(E_{n}^{A+1}-E_{0}^{A}\right)+\mathrm{i} \eta} \\
& +\sum_{k} \frac{\left\langle\Psi_{0}^{A}\left|a_{\beta}^{\dagger}\right| \Psi_{k}^{A-1}\right\rangle\left\langle\Psi_{k}^{A-1}\left|a_{\alpha}\right| \Psi_{0}^{A}\right\rangle}{\hbar \omega-\left(E_{0}^{A}-E_{k}^{A-1}\right)-\mathrm{i} \eta} .
\end{aligned}
$$

In the reminder of this text we will use the following shorthand notation for the quasiparticle energies, given by the poles in Eq. (4),

$$
\begin{aligned}
& \varepsilon_{n}^{+} \equiv\left(E_{n}^{A+1}-E_{0}^{A}\right) \\
& \varepsilon_{k}^{-} \equiv\left(E_{0}^{A}-E_{k}^{A-1}\right),
\end{aligned}
$$

which are the experimentally observed one-nucleon addition and removal energies. Likewise, we will use $\mathcal{X}_{\beta}^{n}$ and $\mathcal{Y}_{\alpha}^{k}$ to mark the transition amplitudes for the addition and removal of a particle, respectively. And we will use $\mathcal{Z}_{\alpha}^{i}$ to collect all of them:

$$
\mathcal{Z}_{\alpha}^{i=n, k} \equiv\left\{\begin{array}{c}
\left(\mathcal{X}_{\alpha}^{n}\right)^{*} \equiv\left\langle\Psi_{0}^{A}\left|a_{\alpha}\right| \Psi_{n}^{A+1}\right\rangle \\
\mathcal{Y}_{\alpha}^{k} \equiv\left\langle\Psi_{k}^{A-1}\left|a_{\alpha}\right| \Psi_{0}^{A}\right\rangle
\end{array}\right.
$$


with the index $i$ referring to both forward-in-time $(n)$ and backward-in-time $(k)$ processes. The first (second) overlap integral in Eq. (6) is related to the probability of adding (removing) a particle to (from) a orbital $\alpha$ in a system with $A$ particles. The vectors $\mathcal{Z}^{i}$ (in the basis $\{\alpha\})$ form the overcomplete set of the eigenstates of the Dyson equation. Thus, they are also referred to as 'Dyson orbitals'.

The 1B Green's function Eq. (4) is completely determined by solving the Dyson equation,

$$
g_{\alpha \beta}(\omega)=g_{\alpha \beta}^{(0)}(\omega)+\sum_{\gamma \delta} g_{\alpha \gamma}^{(0)}(\omega) \Sigma_{\gamma \delta}^{\star}(\omega) g_{\delta \beta}(\omega),
$$

which is a nonlinear equation defining the irreducible self-energy $\Sigma_{\gamma \delta}^{\star}(\omega)$, where medium effects on the particle propagation are encoded. It corresponds to a set of irreducible Feynman diagrams, i.e. diagrams that cannot be divided in sub-diagrams by cutting one propagator line. The distinction between the unperturbed propagator $g_{\alpha \beta}^{(0)}(\omega)$ and the correlated one $g_{\alpha \beta}(\omega)$ in Eq. 77 results from the expansion with respect to the inter-particle interaction: $g_{\alpha \beta}^{(0)}(\omega)$ is then the zeroth-order term in the expansion, that is the propagator with respect to the reference state, i.e.

$$
g_{\alpha \beta}^{(0)}\left(t-t^{\prime}\right)=-\frac{i}{\hbar}\left\langle\phi_{0}^{A}\left|\mathcal{T}\left[a_{\alpha}(t) a_{\beta}^{\dagger}\left(t^{\prime}\right)\right]\right| \phi_{0}^{A}\right\rangle .
$$

From the derivation of the $1 \mathrm{~B}$ propagator equation of motion, one can find the explicit separation of the irreducible self-energy in a part which is local in time $\Sigma_{\alpha \beta}^{\infty}$ (static self-energy), and a energy dependent part $\widetilde{\Sigma}_{\alpha \beta}(\omega)$ (dynamic self-energy), containing the contributions from dynamical excitations in the system:

$$
\Sigma_{\alpha \beta}^{\star}(\omega)=\Sigma_{\alpha \beta}^{\infty}+\widetilde{\Sigma}_{\alpha \beta}(\omega) .
$$

While $\Sigma_{\alpha \beta}^{\infty}$ accounts for the averaged (mean-field) interaction seen by a particle, $\widetilde{\Sigma}_{\alpha \beta}(\omega)$ encodes the coupling of a single-particle state with the collective configurations made by surrounding nucleons. In the continuum regime the full self-energy describes the interaction of the nucleon projectile with a target nucleus. In this respect, $\Sigma_{\alpha \beta}^{\star}(\omega)$ is investigated as the microscopic counterpart of the dispersive optical model potentials [29].

Before proceeding with the application of the ADC formalism to the self-energy, we present in the next section the main features of the approach based on effective interactions, which allows a simplification of the diagrammatic expansion when both two- and many-body forces are included.

\section{A. Formalism with effective forces and interaction-irreducible terms}

Let us consider first the nuclear Hamiltonian $\hat{H}$ with a kinetic energy part $\hat{T}$ and interaction operators in the
2NF and 3NF sector, denoted with $\hat{V}$ and $\hat{W}$ respectively,

$$
\hat{H}=\hat{T}+\hat{V}+\hat{W} .
$$

Within post Hartree-Fock approaches, it is customary to divide the Hamiltonian into two parts, $\hat{H}=\hat{H}_{0}+\hat{H}_{1}$, with $\hat{H}_{0}$ being the uncorrelated part and $\hat{H}_{1}$ the residual interaction. In this way, strongly interacting fermions are treated as a system of independent nucleons affected by an auxiliary mean-field potential $\hat{U}$, included in the definition of $\hat{H}_{0}=\hat{T}+\hat{U}$. The $\hat{H}_{0}$ defines the reference state to which the residual interaction $\hat{H}_{1}$ is added perturbatively. In the second quantization formalism the Hamiltonian reads then,

$$
\begin{aligned}
\hat{H} & =\sum_{\alpha \beta} h_{\alpha \beta}^{(0)} a_{\alpha}^{\dagger} a_{\beta}-\sum_{\alpha \beta} U_{\alpha \beta} a_{\alpha}^{\dagger} a_{\beta}+\frac{1}{4} \sum_{\substack{\alpha \gamma \\
\beta \delta}} V_{\alpha \gamma, \beta \delta} a_{\alpha}^{\dagger} a_{\gamma}^{\dagger} a_{\delta} a_{\beta} \\
& +\frac{1}{36} \sum_{\substack{\alpha \gamma \epsilon \\
\beta \delta \eta}} W_{\alpha \gamma \epsilon, \beta \delta \eta} a_{\alpha}^{\dagger} a_{\gamma}^{\dagger} a_{\epsilon}^{\dagger} a_{\eta} a_{\delta} a_{\beta},
\end{aligned}
$$

where $h_{\alpha \beta}^{(0)} \equiv T_{\alpha \beta}+U_{\alpha \beta}$ is a one-body Hamiltonian and $V_{\alpha \gamma, \beta \delta}$ and $W_{\alpha \gamma \epsilon, \beta \delta \eta}$ are the antisymmetrized matrix elements of $2 \mathrm{NFs}$ and $3 \mathrm{NFs}$, respectively.

The Greek indexes $\alpha, \beta, \gamma, \ldots$ label a complete set of single-particle states which define the model space used in practical computations. In most cases, one chooses this basis as the eigenstates of the unperturbed Hamiltonian $\hat{H}_{0}$, with the eigenvalues $\varepsilon_{\alpha}^{0}$. Then, $h_{\alpha \beta}^{(0)}=\varepsilon_{\alpha}^{0} \delta_{\alpha, \beta}$ and the spectral representation, Eq. (4), for unperturbed propagator $g_{\alpha, \beta}^{(0)}(\omega)$ becomes diagonal. However, in this work we keep the most general case and the basis $\{\alpha\}$ will be different from the one defining the reference state.

The expansion of the self-energy $\widetilde{\Sigma}_{\alpha \beta}(\omega)$ in Eq. $(9)$ involves terms with individual contributions of the $1 \mathrm{~B}$ potential, but also of $2 \mathrm{NFs}$ and $3 \mathrm{NFs}$ from Eq. (11). Of course, also terms containing combinations of different interactions are possible. The number of diagrams allowed by the Feynman diagrammatic rules is growing fast with the order of the expansion. A useful strategy is to consider only interaction-irreducible diagrams. Diagrams are considered interaction-reducible if splitting interaction vertex in two parts results in two disconnected diagrams. This happens when some (but not all) of the fermion lines leaving one interaction vertex eventually return to it. If the interaction vertex which is cut had only one fermionic line looping over it, then all the linked diagrams can be included effectively by averaging the interaction vertex with a 1B Green's function. Alternatively, when the cut interaction vertex had two fermionic lines the averaging is performed with a 2B Green's function and so on. This process of averaging reduces the order of the interaction: for instance, a $2 \mathrm{NF}$ interaction vertex averaged on a 1B Green's function gives rise to an effective $1 \mathrm{~B}$ operator.

In Ref. 22 it is shown that diagrammatic series can be reduced to a smaller set of diagrams by excluding all 
interaction-reducible diagrams. The averaging procedure described above amounts to define an effective Hamiltonian up to $3 \mathrm{NFs}$,

$$
\widetilde{H}_{1}=\widetilde{U}+\widetilde{V}+\hat{W},
$$

where $\widetilde{U}$ and $\widetilde{V}$ represent effective interaction operators. As long as only interaction-irreducible diagrams are considered, the use of $\widetilde{H}_{1}$ is equivalent to the interactionreducible expansion based on Eq. (11) (see Sec. II of Ref. 22] for details).

Explicit expressions for effective $1 \mathrm{~B}$ and $2 \mathrm{~N}$ interaction operators are:

$$
\widetilde{U}=\sum_{\alpha \beta} \widetilde{U}_{\alpha \beta} a_{\alpha}^{\dagger} a_{\beta}
$$

with

$$
\widetilde{U}_{\alpha \beta}=-U_{\alpha \beta}+\sum_{\gamma \delta} V_{\alpha \gamma, \beta \delta} \rho_{\delta \gamma}+\frac{1}{4} \sum_{\substack{\gamma \epsilon \\ \delta \eta}} W_{\alpha \gamma \epsilon, \beta \delta \eta} \Gamma_{\delta \eta, \gamma \epsilon},
$$

and

$$
\widetilde{V}=\frac{1}{4} \sum_{\substack{\alpha \gamma \\ \beta \delta}}\left[V_{\alpha \beta, \gamma \delta}+\sum_{\epsilon \eta} W_{\alpha \beta \epsilon, \gamma \delta \eta} \rho_{\eta \epsilon}\right] a_{\alpha}^{\dagger} a_{\beta}^{\dagger} a_{\delta} a_{\gamma},
$$

where, in the averaging of $2 \mathrm{NFs}$ and $3 \mathrm{NFs}$, one- and twobody reduced density matrices of the many-body system are produced,

$$
\begin{aligned}
\rho_{\delta \gamma} & =\left\langle\Psi_{0}^{A}\left|a_{\gamma}^{\dagger} a_{\delta}\right| \Psi_{0}^{A}\right\rangle=-i \hbar g_{\delta \gamma}\left(t-t^{+}\right), \\
\Gamma_{\delta \eta, \gamma \epsilon} & =\left\langle\Psi_{0}^{A}\left|a_{\gamma}^{\dagger} a_{\epsilon}^{\dagger} a_{\eta} a_{\delta}\right| \Psi_{0}^{A}\right\rangle=i \hbar g_{\delta \eta, \gamma \epsilon}^{I I}\left(t-t^{+}\right) .
\end{aligned}
$$

The two-body density of Eq. (17) is obtained when the opportune limits are taken in the time arguments of the 2B Green's function in Eq. (2).

We note that when the irreducible self-energy is computed with the effective Hamiltonian of Eq. (12), a portion of the many-body effects is incorporated in the interactions, which become system dependent. This is done in a systematic way and the procedure is in principle superior to the usual normal ordering approach. Here the density matrices $\rho$ and $\Gamma$ entering the contraction of the interaction vertex are obtained from the true correlated propagators, i.e. they are not computed from the reference state.

The separation of a simple unperturbed Hamiltonian $\hat{H}_{0}$ from Eq. 111 is instrumental to any approach based on perturbation theory (or on all-orders resummations): it allows us to define a reference state upon which a perturbative series is constructed and it also leads to the expansion of the Green's function in Feynman diagrams. Nevertheless, the auxiliary potential $\hat{U}$ eventually cancels from the SCGF formalism. Considering the decomposition of Eq. (9), the irreducible static self-energy $\Sigma_{\alpha \beta}^{\infty}$ is given exactly by the $1 \mathrm{~B}$ effective interaction [22]:

$$
\Sigma_{\alpha \beta}^{\infty}=\widetilde{U}_{\alpha \beta} .
$$

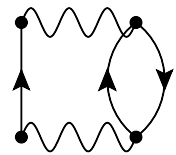

(a)

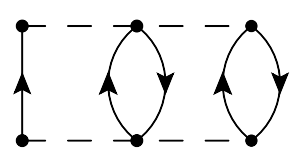

(b)
FIG. 1. One-particle irreducible, skeleton and interactionirreducible self-energy diagrams appearing at second order in the expansion of Eq. (9), using the effective Hamiltonian of Eq. (12). The wiggly lines represent the $2 \mathrm{~N}$ effective interaction of Eq. 15, while the long-dashed lines represent the interaction-irreducible $3 \mathrm{NF} \hat{W}$.

Since $\hat{U}$ is added to the definition of the reference propagator $g^{(0)}$ but subtracted in Eq. 14, it eventually cancels out exactly from the Dyson equation (see Eq. (28p). The dynamic self-energy $\widetilde{\Sigma}_{\alpha \beta}(\omega)$ can still depend on the auxiliary potential through the perturbative expansion in $g_{\alpha \beta}^{(0)}(\omega)$. However, in the full self-consistent approach, the perturbative series is restricted to skeleton diagrams where fully correlated propagators $g_{\alpha \beta}(\omega)$ replace the uncorrelated ones. Thus, the partition of the Hamiltonian into a uncorrelated part and residual part is completely lost in the exact SCGF formalism and one may think of the correlated propagator as playing the role of an improved reference state.

For the irreducible self-energy, all one-particle irreducible, skeleton and interaction-irreducible diagrams up to third order have been derived in Ref. [22. Within the skeleton expansion, i.e. when single-particle propagators are correlated, the irreducible self-energy up to the third order is given by the exact static part, Eq. 18, the two second-order diagrams of Fig. 1, and the 17 third-order diagrams of Figs. 2 and 6 . In this case, the energy-dependent part of the self-energy contains only effective 2NFs and irreducible 3NFs as interaction insertions. Note that because of Eq. (15), the contribution of Fig. 1a actually corresponds to four separate diagrams if expressed in terms of the bare Hamiltonian (10), of which three are interaction reducible 22. Likewise, many more reducible diagrams would appear at third order. Without propagator renormalization, when one considers the diagrammatic expansion with reference propagators $g_{\alpha \beta}^{(0)}(\omega)$ as internal fermionic lines, other diagrams with different topologies must be included to take into account explicitly additional correlations in both the static and dynamic part of the self-energy. These terms contain also nonskeleton diagrams that include $\widetilde{U}$ and are presented in Appendix C.

In Fig. 1 we show the only two one-particle irreducible, skeleton and interaction-irreducible diagrams at second order. These diagrams imply different sets of intermediate state configurations (ISCs), which are multiparticle-multihole, or multihole-multiparticle, excitations produced by the interaction and propagating within the nuclear medium. The diagram in Fig. 1a involves two-particles-one-hole $(2 p 1 h)$ and two-holes-one- 


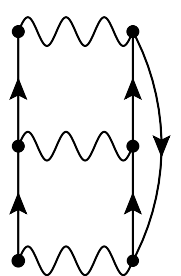

(a)

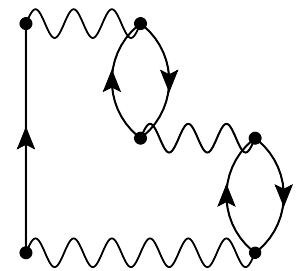

(b)

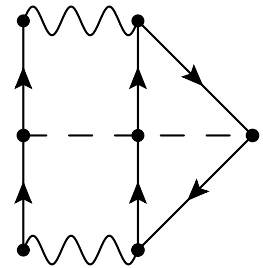

(c)
FIG. 2. As described in the caption of Fig. 1 but for the third-order diagrams with only $2 p 1 h$ and $2 h 1 p$ intermediate state configurations.

particle $(2 h 1 p)$ ISCs, but it is computed with the $2 \mathrm{~N}$ effective interaction Eq. (15) instead of the original 2NF. Hence, it contains contributions form the $3 \mathrm{NF} \hat{W}$.

The diagram in Fig. 1b arises instead from an interaction-irreducible $3 \mathrm{NF}$. There are two reasons to assume that this contribution is less important than the one in Fig. 1a first, 3NFs are generally weaker than corresponding 2NFs (typically, $\left\langle\widehat{W}>\approx \frac{1}{10}<\widehat{V}>\right.$ for nuclear interactions [8, 30]); second, the diagram in Fig. 1b involves $3 p 2 h$ and $3 h 2 p$ ISCs, which involve higher excitation energies and therefore they are expected to play a minor role at the Fermi surface due to phase space arguments.

By the same token, we may expect that the three diagrams shown in Fig. (2) are the dominant ones among the 17 one-particle irreducible, skeleton, and interactionirreducible self-energy diagrams appearing at third order in the expansion of $\widetilde{\Sigma}_{\alpha \beta}(\omega)$. While all diagrams in Fig. (2) involve $2 p 1 h$ and $2 h 1 p$ ISCs, those in Figs. 2a and $2 \mathrm{~b}$ contain only effective $2 \mathrm{NF}$. These two diagrams have been already included in actual calculations for nuclear matter and for finite nuclei [3, 24, 25, 31, 33.

\section{B. Solution of the Dyson equation as a matrix eigenvalue problem}

The ADC is a systematic approach to calculate nonperturbative approximations to the self-energy. Once the latter is known, we still need to solve the Dyson equation to obtain the propagator, as explained below.

Without loss of generality, the dynamical part of the self-energy $\widetilde{\Sigma}_{\alpha \beta}(\omega)$ can be written in the Lehmann rep- resentation, similar to Eq. (4). Specifically, we write,

$$
\begin{aligned}
\widetilde{\Sigma}_{\alpha \beta}(\omega) & =\sum_{j j^{\prime}} \mathbf{M}_{\alpha j}^{\dagger}\left[\frac{1}{\hbar \omega \mathbb{1}-\left(\mathbf{E}^{>}+\mathbf{C}\right)+\mathrm{i} \eta \mathbb{1}}\right]_{j j^{\prime}} \mathbf{M}_{j^{\prime} \beta} \\
& +\sum_{k k^{\prime}} \mathbf{N}_{\alpha k}\left[\frac{1}{\hbar \omega \mathbb{1}-\left(\mathbf{E}^{<}+\mathbf{D}\right)-\mathrm{i} \eta \mathbb{1}}\right]_{k k^{\prime}} \mathbf{N}_{k^{\prime} \beta}^{\dagger},
\end{aligned}
$$

with $\mathbf{E}_{j j^{\prime}}^{>}\left(\mathbf{E}_{k k^{\prime}}^{<}\right)$being energies of noninteracting ISCs, $\mathbf{M}_{j \alpha}\left(\mathbf{N}_{\alpha k}\right)$ coupling matrices, and $\mathbf{C}_{j j^{\prime}}\left(\mathbf{D}_{k k^{\prime}}\right)$ interaction matrices for the forward-in-time (backwardin-time) self-energy. Coupling matrices link initial and final single-particle states of the propagator to ISCs, while interaction matrices are those parts of the self-energy diagrams that represent interactions among ISCs alone (see also Fig. 3). It follows that interaction matrices contain at most one interaction vertex and are not linked to the single-particle states of the model space. We use Latin letters as a collective indices to label ISCs: In particular, we use $i$ for any general configuration, while $j, j^{\prime}\left(k, k^{\prime}\right)$ denote forward-in-time multiparticle-multihole (backwardin-time multihole-multiparticle) configurations. In the following, we will consider explicitly $2 p 1 h, 3 p 2 h, 2 h 1 p$, and $3 h 2 p$ ISCs, which are included in Eq. 19.). For this purpose, we set the shorthand notation,

$$
\left.\begin{array}{rl}
r & \equiv\left(n_{1}, n_{2}, k_{3}\right) \\
r^{\prime} & \equiv\left(n_{4}, n_{5}, k_{6}\right) \\
q & \equiv\left(n_{1}, n_{2}, n_{3}, k_{4}, k_{5}\right) \\
q^{\prime} & \equiv\left(n_{6}, n_{7}, n_{8}, k_{9}, k_{10}\right)
\end{array}\right\} j, j^{\prime}
$$

for forward-in-time terms, and

$$
\left.\begin{array}{rl}
s & \equiv\left(k_{1}, k_{2}, n_{3}\right) \\
s^{\prime} & \equiv\left(k_{4}, k_{5}, n_{6}\right) \\
u & \equiv\left(k_{1}, k_{2}, k_{3}, n_{4}, n_{5}\right) \\
u^{\prime} & \equiv\left(k_{6}, k_{7}, k_{8}, n_{9}, n_{10}\right)
\end{array}\right\} k, k^{\prime}
$$

for backward-in-time terms, where $n_{i}\left(k_{i}\right)$ refer to the particle (hole) indices of the propagator, Eq. (4). For instance, $\mathbf{M}_{r \alpha} \equiv \mathbf{M}_{\left(n_{1}, n_{2}, k_{3}\right) \alpha}$ connects a single-particle state of index $\alpha$ to an intermediate state composed by a $2 p 1 h$ configuration, whereas more complicated coupling matrices such as $\mathbf{M}_{q \alpha} \equiv \mathbf{M}_{\left(n_{1}, n_{2}, n_{3}, k_{4}, k_{5}\right) \alpha}$ involve $3 p 2 h$ configurations. As one moves to higher orders beyond ADC(3) more complex multi-particle-multi-hole states appear in Eq. (19), eventually covering the complete space of ISCs.

To better clarify how the ADC building blocks are associated to perturbation theory, we show in Fig. 3 the diagrammatic decomposition for two of the Goldstone contributions that arise from the self-energy Feynman diagram of Fig. 2c. The expressions for the coupling and interactions matrices can be read directly form the analytic expression of each Goldstone diagram. See Ref. 2] for a detailed pedagogical discussion. 


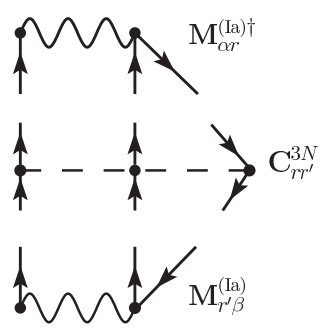

(a)
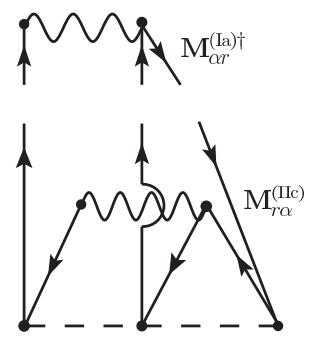

(b)
FIG. 3. Decomposition of Goldstone diagrams in terms of interaction and coupling matrices. Two (out of the six) Goldstone diagrams that arise from the self-energy Feynman diagram of Fig. 2c are shown. Diagram (a) contains the interaction matrix $\mathbf{C}_{r r^{\prime}}^{3 N}$ (see Eq. 63) ) linked to two lowest order coupling matrices $\mathbf{M}_{r \alpha}^{(\mathrm{Ia})}$ (see Eq. (34)). Diagram (b) contains only coupling matrices and includes the second-order correction $\mathbf{M}_{r \alpha}^{(\mathrm{IIc})}$ reported in Eq. 57.

In virtue of the Pauli principle, the expressions for coupling and interaction matrices can (and should) be made antisymmetric with respect to permutation of any two particle or any two hole indexes. This results naturally from the antisymmetry of the interaction matrix elements, Eq. (11), and by including a complete set of diagrams at each order, which generates all possible permutations [21, 34]. Using the antisymmetry, it is possible to restrict the sums in Eq. 19 to sets of ordered single-particle indices, defining for instance $\left(n_{1}<n_{2}, k_{3}\right)$, $\left(n_{1}<n_{2}<n_{3}, k_{4}<k_{5}\right)$, and so on. This is very important for practical implementations, owing to a substantial reduction of the dimension of the Dyson matrix, Eq. (28) below. On the other hand, manipulations such as coupling the angular momenta of ISCs is better performed in the general case of unrestricted summations. Therefore, we will present all the working equations without assuming ordered indexes, as given by the notation of Eqs. (20) and (21). One can always apply ordered summation by removing the relevant symmetry factors to the expressions given in Sec. III and in the Appendices.

ISC energies are diagonal matrices in these indexes. For nucleon addition, with $M+1$ particles and $M$ holes, $(M+1) p M h$, we have,

$$
\begin{aligned}
\mathbf{E}_{j j^{\prime}}^{>}=\mathbf{E}_{j}^{>}= & \operatorname{diag}\left(\varepsilon_{n_{1}}^{+}+\varepsilon_{n_{2}}^{+}+\cdots+\varepsilon_{n_{M}}^{+}+\varepsilon_{n_{M+1}}^{+}\right. \\
& \left.-\varepsilon_{k_{1}}^{-}-\varepsilon_{k_{2}}^{-}-\cdots-\varepsilon_{k_{M}}^{-}\right),
\end{aligned}
$$

while for the nucleon removal ISC,

$$
\begin{aligned}
\mathbf{E}_{k k^{\prime}}^{<}=\mathbf{E}_{k}^{<}= & \operatorname{diag}\left(\varepsilon_{k_{1}}^{-}+\varepsilon_{k_{2}}^{-}+\cdots+\varepsilon_{k_{M}}^{-}+\varepsilon_{k_{M+1}}^{-}\right. \\
& \left.-\varepsilon_{n_{1}}^{+}-\varepsilon_{n_{2}}^{+}-\cdots-\varepsilon_{n_{M}}^{+}\right) .
\end{aligned}
$$

To solve for the 1B propagator of Eq. (4), it is useful to recast the Dyson equation in matrix form, which allows a more efficient computation of $1 \mathrm{~B}$ propagator eigenvalues. To see this point, we have to start again from the Dyson equation (7) and regard the 1B propagator Eq. (4) as a meromorphic function on the complex energy plane. This function has simple poles and residues given by onenucleon addition (or removal) energies and transition amplitudes, respectively. We can then find a relation among the transition amplitudes of Eq. (6), by extracting them as residues of the propagator in the Dyson equation. This gives,

$$
\mathcal{Z}_{\alpha}^{i}\left(\mathcal{Z}_{\beta}^{i}\right)^{\dagger}=\left.\sum_{\gamma \delta}\left[\frac{1}{\hbar \omega \mathbb{1}-\hat{H}_{0}}\right]_{\alpha \gamma} \Sigma_{\gamma \delta}^{*}(\omega) \mathcal{Z}_{\delta}^{i}\left(\mathcal{Z}_{\beta}^{i}\right)^{\dagger}\right|_{\hbar \omega=\varepsilon_{i}} .
$$

By using the decomposition of Eq. (19) we obtain the relation,

$$
\mathcal{Z}_{\alpha}^{i}=\left.\sum_{\gamma \delta}\left[\frac{1}{\hbar \omega \mathbb{1}-\hat{H}_{0}}\right]_{\alpha \gamma} \Sigma_{\gamma \delta}^{*}(\omega) \mathcal{Z}_{\delta}^{i}\right|_{\hbar \omega=\varepsilon_{i}}=\left.\sum_{\gamma}\left[\frac{1}{\hbar \omega \mathbb{1}-\hat{H}_{0}}\right]_{\alpha \gamma}\left(\sum_{\delta} \Sigma_{\gamma \delta}^{\infty} \mathcal{Z}_{\delta}^{i}+\sum_{j} \mathbf{M}_{\gamma j}^{\dagger} \mathcal{W}_{j}^{i}+\sum_{k} \mathbf{N}_{\gamma k} \mathcal{W}_{k}^{i}\right)\right|_{\hbar \omega=\varepsilon_{i}}
$$

which contains both forward-in-time and backward-in-time solutions of the propagator. In the last equality of Eq. (25) we have introduced the vectors $\mathcal{W}_{j}^{i}$ and $\mathcal{W}_{k}^{i}$, defined as

$$
\left.\mathcal{W}_{j}^{i} \equiv \mathcal{W}_{j}(\omega)\right|_{\hbar \omega=\varepsilon_{i}}=\left.\sum_{j^{\prime}}\left[\frac{1}{\hbar \omega \mathbb{1}-\left(\mathbf{E}^{>}+\mathbf{C}\right)}\right] \sum_{j j^{\prime}} \mathbf{M}_{j^{\prime} \delta} \mathcal{Z}_{\delta}^{i}\right|_{\hbar \omega=\varepsilon_{i}}
$$

and

$$
\left.\mathcal{W}_{k}^{i} \equiv \mathcal{W}_{k}(\omega)\right|_{\hbar \omega=\varepsilon_{i}}=\left.\sum_{k^{\prime}}\left[\frac{1}{\hbar \omega \mathbb{1}-\left(\mathbf{E}^{<}+\mathbf{D}\right)}\right]_{k k^{\prime}} \sum_{\delta} \mathbf{N}_{k^{\prime} \delta}^{\dagger} \mathcal{Z}_{\delta}^{i}\right|_{\hbar \omega=\varepsilon_{i}}
$$

respectively. These vectors are introduced to recast the Dyson equation as a large scale eigenvalue problem whose diagonalization gives the eigenspectra of the $\left|\Psi_{n}^{A+1}\right\rangle$ and $\left|\Psi_{k}^{A-1}\right\rangle$ systems and the transition amplitudes of the 1B 
propagator. By recoupling Eqs. 25)-27), we obtain:

$$
\epsilon_{i}\left(\begin{array}{c}
\mathcal{Z}_{\alpha}^{i} \\
\mathcal{W}_{r}^{i} \\
\mathcal{W}_{s}^{i} \\
\mathcal{W}_{q}^{i} \\
\mathcal{W}_{u}^{i} \\
\vdots
\end{array}\right)=\left(\begin{array}{cccccc}
h_{\alpha \delta}^{(0)}+\Sigma_{\alpha \delta}^{\infty} & \mathbf{M}_{\alpha r^{\prime}}^{\dagger} & \mathbf{N}_{\alpha s^{\prime}} & \mathbf{M}_{\alpha q^{\prime}}^{\dagger} & \mathbf{N}_{\alpha u^{\prime}} & \cdots \\
\mathbf{M}_{r \delta} & \mathbf{E}_{r}^{>} \delta_{r r^{\prime}}+\mathbf{C}_{r r^{\prime}} & 0 & \mathbf{C}_{r q^{\prime}} & 0 & \ldots \\
\mathbf{N}_{s \delta}^{\dagger} & 0 & \mathbf{E}_{s}^{<} \delta_{s s^{\prime}}+\mathbf{D}_{s s^{\prime}} & 0 & \mathbf{D}_{s u^{\prime}} & \cdots \\
\mathbf{M}_{q \delta} & \mathbf{C}_{q r^{\prime}} & 0 & \mathbf{E}_{q}^{>} \delta_{q q^{\prime}}+\mathbf{C}_{q q^{\prime}} & 0 & \ldots \\
\mathbf{N}_{u \delta}^{\dagger} & 0 & \mathbf{D}_{u s^{\prime}} & 0 & \mathbf{E}_{u}^{<} \delta_{u u^{\prime}}+\mathbf{D}_{u u^{\prime}} & \cdots \\
\vdots & \vdots & \vdots & \vdots & \vdots
\end{array}\right)\left(\begin{array}{c}
\mathcal{Z}_{\delta}^{i} \\
\mathcal{W}_{r^{\prime}}^{i} \\
\mathcal{W}_{s^{\prime}}^{i} \\
\mathcal{W}_{q^{\prime}}^{i} \\
\mathcal{W}_{u^{\prime}}^{i} \\
\vdots
\end{array}\right),
$$

where the eigenmatrix is referred to as 'Dyson matrix' and it is independent of $\hbar \omega$. The zero entries mean that the forward-in-time and backward-in-time sectors are coupled together only through single particle states, and the dots stay for the self-energy terms with ISCs beyond the $3 p 2 h$ and $3 h 2 p$ configurations.

Equation (28) is an energy-independent eigenvalue problem, whereas the components $\mathcal{W}^{i}$ of the eigenvectors are functions of the corresponding eigenvalue $\varepsilon_{i}$, as it is apparent from definitions (26 27). The diagonalization of the Dyson matrix yields all the poles of the propagator at once, while the normalization of the $i$-th eigenvector is given by

$$
\sum_{\alpha}\left|\mathcal{Z}_{\alpha}^{i}\right|^{2}+\sum_{j}\left|\mathcal{W}_{j}^{i}\right|^{2}+\sum_{k}\left|\mathcal{W}_{k}^{i}\right|^{2}=1
$$

In a self-consistent calculation, the elements of the Dyson matrix Eq. (28) depend on the quasiparticle energies and amplitudes, $\varepsilon_{i}$ and $\mathcal{Z}_{\alpha}^{i}$. Thus, they require an iterative solution. The large number of poles in the dressed propagator, see Eq. (4), implies a severe growth in the dimension of the Dyson matrix at each iteration, as explained in Sec. IIIA of Ref. 35. This can be handled by projecting the space of intermediate configurations into smaller Krylov subspaces, using Lanczos-type algorithms with multiple pivots [35].

\section{GENERAL OUTLINE OF THE ADC $(n)$ METHOD}

The irreducible self-energy $\Sigma_{\alpha \beta}^{\star}(\omega)$ is the object of the ADC formalism applied in this work. Its expression as a product of matrices, Eqs. (9) and (19), is the most general analytic form that is consistent with the causality principle and the known Lehmann representation.

Our task is then to find expressions for coupling and interaction matrices including the correlations due to 2NFs and 3NFs. The $\operatorname{ADC}(n)$ strategy consists in deriving explicit expressions of the coupling and interaction matrices by expanding Eq. 19 in powers of $2 \mathrm{NFs}$ and $3 \mathrm{NFs}$ and then to compare with the Goldstone-Feynman expansion up to order $n$. Formally, we have

$$
\mathbf{M}_{j \alpha}=\mathbf{M}_{j \alpha}^{(\mathrm{I})}+\mathbf{M}_{j \alpha}^{(\mathrm{II})}+\mathbf{M}_{j \alpha}^{(\mathrm{III})}+\ldots
$$

where the term $\mathbf{M}_{j \alpha}^{(\mathrm{n})}$ is of nth order in the residual Hamiltonian $H_{1}$, and similarly for backward-in-time coupling matrices:

$$
\mathbf{N}_{\alpha k}=\mathbf{N}_{\alpha k}^{(\mathrm{I})}+\mathbf{N}_{\alpha k}^{(\mathrm{II})}+\mathbf{N}_{\alpha k}^{(\mathrm{III})}+\ldots
$$

By plugging Eqs. (30) and 31) into Eq. (19), we obtain the corresponding expansion for the energy-dependent irreducible self-energy up to third order (first order contributions are all included in $\Sigma_{\alpha \beta}^{\infty}$ ). This is 


$$
\begin{aligned}
\widetilde{\Sigma}_{\alpha \beta}(\omega)= & \sum_{j} \mathbf{M}_{\alpha j}^{(\mathrm{I}) \dagger}\left[\frac{1}{\hbar \omega-\mathbf{E}_{j}^{>}+\mathrm{i} \eta}\right] \mathbf{M}_{j \beta}^{(\mathrm{I})} \\
& +\sum_{j} \mathbf{M}_{\alpha j}^{(\mathrm{II}) \dagger}\left[\frac{1}{\hbar \omega-\mathbf{E}_{j}^{>}+\mathrm{i} \eta}\right] \mathbf{M}_{j \beta}^{(\mathrm{I})}+\sum_{j} \mathbf{M}_{\alpha j}^{(\mathrm{I}) \dagger}\left[\frac{1}{\hbar \omega-\mathbf{E}_{j}^{>}+\mathrm{i} \eta}\right] \mathbf{M}_{j \beta}^{(\mathrm{II})} \\
& +\sum_{j j^{\prime}} \mathbf{M}_{\alpha j}^{(\mathrm{I}) \dagger}\left[\frac{1}{\hbar \omega-\mathbf{E}_{j}^{>}+\mathrm{i} \eta}\right] \mathbf{C}_{j j^{\prime}}\left[\frac{1}{\hbar \omega-\mathbf{E}_{j^{\prime}}^{>}+\mathrm{i} \eta}\right] \mathbf{M}_{j^{\prime} \beta}^{(\mathrm{I})}+\ldots \\
& +\sum_{k} \mathbf{N}_{\alpha k}^{(\mathrm{I})}\left[\frac{1}{\hbar \omega-\mathbf{E}_{k}^{<}-\mathrm{i} \eta}\right] \mathbf{N}_{k \beta}^{(\mathrm{I}) \dagger} \\
& +\sum_{k} \mathbf{N}_{\alpha k}^{(\mathrm{II})}\left[\frac{1}{\hbar \omega-\mathbf{E}_{k}^{<}-\mathrm{i} \eta}\right] \mathbf{N}_{k \beta}^{(\mathrm{I}) \dagger}+\sum_{k} \mathbf{N}_{\alpha k}^{(\mathrm{I})}\left[\frac{1}{\hbar \omega-\mathbf{E}_{k}^{<}-\mathrm{i} \eta}\right] \mathbf{N}_{k \beta}^{(\mathrm{II}) \dagger} \\
& +\sum_{k k^{\prime}} \mathbf{N}_{\alpha k}^{(\mathrm{I})}\left[\frac{1}{\hbar \omega-\mathbf{E}_{k}^{<}-\mathrm{i} \eta}\right] \mathbf{D}_{k k^{\prime}}\left[\frac{1}{\hbar \omega-\mathbf{E}_{k^{\prime}}^{<}-\mathrm{i} \eta}\right] \mathbf{N}_{k^{\prime} \beta}^{(\mathrm{I}) \dagger}+\ldots
\end{aligned}
$$

for both forward-in-time and backward-in-time selfenergy parts. The comparison of the formal expansion of Eq. 32 with the calculated Goldstone-type diagrams, gives the minimal expressions for interaction and coupling matrices in terms of the transition amplitudes $\mathcal{Z}_{\alpha}^{i}$ and the one-nucleon addition $\varepsilon_{n}^{+}$and removal $\varepsilon_{k}^{-}$energies of the 1 B propagator $g_{\alpha \beta}(\omega)$ of Eq. (4). By looking at the expansion in Eq. (32), we see that the third-order terms containing the interaction matrices $\mathbf{C}_{j j^{\prime}}$ and $\mathbf{D}_{k k^{\prime}}$ do not retain the same analytic form as Eq. (19), which is based on the Lehmann representation of the propagator itself. To recover this analytic form in terms of self-energy poles, one must introduce higher-order terms and perform a resummation of those diagrams up to infinite order: this resummation is implicit in Eq. 28) and it gives the nonperturbative character of the method, which takes into account at all orders several types of diagrams, particleparticle and hole-hole ladders, and particle-hole rings, as well as other resummations induced by 3NFs.

\section{A. ADC method at second order: $\operatorname{ADC}(2)$}

In this section we present the explicit expressions of coupling and interaction matrices entering the $\mathrm{ADC}(2)$ formalism. The two second order diagrams shown in
Fig. 1 are sufficient to define the $\operatorname{ADC}(2)$ approximation scheme. Coupling and interaction matrices required to build the $\mathrm{ADC}(3)$ are introduced in Sec. IIIB and Appendix A. Unless otherwise stated, for coupling and interaction matrices we adopt the Einstein's convention of summing over repeated indices for both the modelspace single-particle states $(\alpha, \beta, \ldots)$ and the particle and hole orbits $\left(n_{1}, n_{2}, \ldots, k_{1}, k_{2} \ldots\right)$. We also use collective indexes for ISCs according to the notation set in Eqs. 20 and 21, where appropriate.

We have seen that for a given multiparticle-multihole or multihole-multiparticle configuration, we can have coupling matrices at different orders according to the expansions in Eqs. (30) and (31). Within a given order, coupling matrices can also differ with respect to the kind of interaction ( $2 \mathrm{NF}$ and/or $3 \mathrm{NF}$ ) appearing in the term. For this reason we specify in the notation an extra superscript distinguishing different contributions at the same order. For instance, at second order we will encounter a coupling matrix $\mathbf{M}_{r \alpha}^{\text {(Ia) }}$ containing a $2 \mathrm{~N}$ interaction linked to a $r=\left(n_{1}, n_{2}, k_{3}\right)$ ISC, $\mathbf{M}_{q \alpha}^{(\mathrm{Ib})}$ containing a $3 \mathrm{~N}$ interaction linked to a $q=\left(n_{1}, n_{2}, n_{3}, k_{4}, k_{5}\right)$ ISC, and so on. The extra superscript with Latin letter corresponds to the labels of diagrams in the figures.

To illustrate the ADC procedure, we write first the entire expressions for all the Goldstone terms in each second order Feynman diagram of Fig. 1. Then we display the formulas of the coupling matrices that can be singled out from the self-energy expressions. The equation for the dynamic self-energy in Fig. 1a reads,

$$
\widetilde{\Sigma}_{\alpha \beta}^{(1 a)}(\omega)=\frac{1}{2} \widetilde{V}_{\alpha \epsilon, \gamma \rho}\left(\sum_{\substack{n_{1}, n_{2} \\ k_{3}}} \frac{\left(\mathcal{X}_{\gamma}^{n_{1}} \mathcal{X}_{\rho}^{n_{2}} \mathcal{Y}_{\epsilon}^{k_{3}}\right)^{*} \mathcal{X}_{\mu}^{n_{1}} \mathcal{X}_{\nu}^{n_{2}} \mathcal{Y}_{\lambda}^{k_{3}}}{\hbar \omega-\left(\varepsilon_{n_{1}}^{+}+\varepsilon_{n_{2}}^{+}-\varepsilon_{k_{3}}^{-}\right)+i \eta}+\sum_{\substack{k_{1}, k_{2} \\ n_{3}}} \frac{\mathcal{Y}_{\gamma}^{k_{1}} \mathcal{Y}_{\rho}^{k_{2}} \mathcal{X}_{\epsilon}^{n_{3}}\left(\mathcal{Y}_{\mu}^{k_{1}} \mathcal{Y}_{\nu}^{k_{2}} \mathcal{X}_{\lambda}^{n_{3}}\right)^{*}}{\hbar \omega-\left(\varepsilon_{k_{1}}^{-}+\varepsilon_{k_{2}}^{-}-\varepsilon_{n_{3}}^{+}\right)-i \eta}\right) \widetilde{V}_{\mu \nu, \beta \lambda}
$$

Being already in the Lehmann form of Eq. [19, we can read directly from Eq. (33) the forward-in-time contribution 
to the $\mathrm{ADC}(2)$ coupling matrix,

$$
\mathbf{M}_{r \alpha}^{(\mathrm{Ia})} \equiv \frac{1}{\sqrt{2}} \mathcal{X}_{\mu}^{n_{1}} \mathcal{X}_{\nu}^{n_{2}} \mathcal{Y}_{\lambda}^{k_{3}} \widetilde{V}_{\mu \nu, \alpha \lambda}
$$

while in the backward-in-time channel we have

$$
\mathbf{N}_{\alpha s}^{(\mathrm{Ia})} \equiv \frac{1}{\sqrt{2}} \widetilde{V}_{\alpha \lambda, \mu \nu} \mathcal{Y}_{\mu}^{k_{1}} \mathcal{Y}_{\nu}^{k_{2}} \mathcal{X}_{\lambda}^{n_{3}},
$$

that couples the effective $2 \mathrm{NF}$ with the $2 h 1 p$ ISC. It is also clear that the interaction matrices $\mathbf{C}_{j j^{\prime}}$ and $\mathbf{D}_{k k^{\prime}}$ are zero in $\mathrm{ADC}(2)$. The representations of Eqs. (34) and (35) as fragments of Goldstone diagrams are depicted in Figs. $4 \mathrm{a}$ and 5a, respectively.

The equation for the energy-dependent self-energy with 3NFs in Fig. 1 b reads,

$$
\begin{aligned}
\widetilde{\Sigma}_{\alpha \beta}^{(1 b)}(\omega)=\frac{1}{12} W_{\alpha \gamma \delta, \xi \tau \sigma} & \left(\sum_{\substack{n_{1}, n_{2}, n_{3} \\
k_{4}, k_{5}}} \frac{\left(\mathcal{X}_{\xi}^{n_{1}} \mathcal{X}_{\tau}^{n_{2}} \mathcal{X}_{\sigma}^{n_{3}} \mathcal{Y}_{\gamma}^{k_{4}} \mathcal{Y}_{\delta}^{k_{5}}\right)^{*} \mathcal{X}_{\mu}^{n_{1}} \mathcal{X}_{\nu}^{n_{2}} \mathcal{X}_{\lambda}^{n_{3}} \mathcal{Y}_{\eta}^{k_{4}} \mathcal{Y}_{\rho}^{k_{5}}}{\hbar \omega-\left(\varepsilon_{n_{1}}^{+}+\varepsilon_{n_{2}}^{+}+\varepsilon_{n_{3}}^{+}-\varepsilon_{k_{4}}^{-}-\varepsilon_{k_{5}}^{-}\right)+i \eta}\right. \\
& \left.+\sum_{\substack{k_{1}, k_{2}, k_{3} \\
n_{4}, n_{5}}} \frac{\mathcal{Y}_{\xi}^{k_{1}} \mathcal{Y}_{\tau}^{k_{2}} \mathcal{Y}_{\sigma}^{k_{3}} \mathcal{X}_{\gamma}^{n_{4}} \mathcal{X}_{\delta}^{n_{5}}\left(\mathcal{Y}_{\mu}^{k_{1}} \mathcal{Y}_{\nu}^{k_{2}} \mathcal{Y}_{\lambda}^{k_{3}} \mathcal{X}_{\eta}^{n_{4}} \mathcal{X}_{\rho}^{n_{5}}\right)^{*}}{\hbar \omega-\left(\varepsilon_{k_{1}}^{-}+\varepsilon_{k_{2}}^{-}+\varepsilon_{k_{3}}^{-}-\varepsilon_{n_{4}}^{+}-\varepsilon_{n_{5}}^{+}\right)-i \eta}\right) W_{\mu \nu \lambda, \beta \eta \rho} .
\end{aligned}
$$

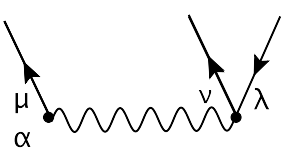

(a)

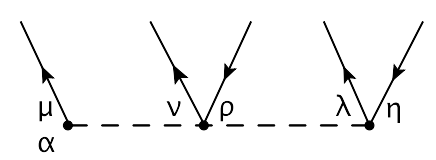

(b)

FIG. 4. Diagrams of the self-energy coupling matrices with the effective $2 \mathrm{NF} \widetilde{V}$ (left) and with interaction-irreducible $3 \mathrm{NF} \hat{W}$ (right). The coupling matrix (a) connects to the $2 p 1 h$ ISCs (see Eq. (34)), while the coupling matrix (b) connects to the $3 p 2 h$ ISCs (see Eq. (37)).

The coupling matrix that links the $3 \mathrm{NF}$ to $3 p 2 h$ ISCs is found in the diagram of Fig. $1 \mathrm{~b}$ and it is read from Eq. (36). Its expression is,

$$
\mathbf{M}_{q \alpha}^{(\mathrm{Ib})} \equiv \frac{1}{\sqrt{12}} \mathcal{X}_{\mu}^{n_{1}} \mathcal{X}_{\nu}^{n_{2}} \mathcal{X}_{\lambda}^{n_{3}} \mathcal{Y}_{\rho}^{k_{4}} \mathcal{Y}_{\eta}^{k_{5}} W_{\mu \nu \lambda, \alpha \rho \eta},
$$

while the corresponding matrix linked to $3 h 2 p$ ISCs is,

$$
\mathbf{N}_{\alpha u}^{(\mathrm{Ib})} \equiv \frac{1}{\sqrt{12}} W_{\alpha \rho \eta, \mu \nu \lambda} \mathcal{Y}_{\mu}^{k_{1}} \mathcal{Y}_{\nu}^{k_{2}} \mathcal{Y}_{\lambda}^{k_{3}} \mathcal{X}_{\rho}^{n_{4}} \mathcal{X}_{\eta}^{n_{5}}
$$

Equation (38) is also found in the diagram of Fig. $1 \mathrm{~b}$ and in the second term of Eq. (36). Their representations as fragments of Goldstone diagrams are depicted in Figs. 4b and $5 \mathrm{~b}$,

The four coupling matrices in Eqs. (34), (35) and (37), (38), along with their complex conjugates, complete the set of matrices found in the irreducible Goldstone diagrams of the self-energy at second order, which are given by the first and fourth rows in Eq. (32). All these matrices enter as building blocks of the ADC construction

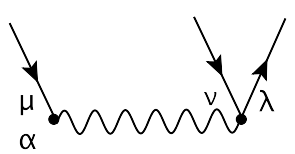

(a)

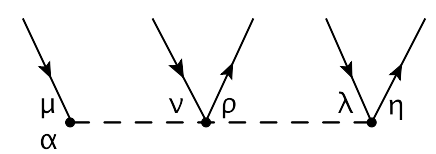

(b)
FIG. 5. As in Fig. 4 but for backward-in-time coupling matrices (see Eqs. (35) and (38) respectively).

at second and third order in the expansion with respect to the nuclear interaction. To summarize, the $\operatorname{ADC}(2)$ approximation for Eq. 19 requires the following terms,

$$
\begin{aligned}
& \mathbf{M}_{j \alpha}^{[\mathrm{ADC}(2)]}= \begin{cases}\mathbf{M}_{r \alpha}^{(\mathrm{Ia})} & \text { if } j=r(2 p 1 h), \\
\mathbf{M}_{q \alpha}^{\mathrm{Ib}} & \text { if } j=q(3 p 2 h),\end{cases} \\
& \mathbf{N}_{\alpha k}^{[\mathrm{ADC}(2)]}= \begin{cases}\mathbf{N}_{\alpha s}^{(\mathrm{Ia})} & \text { if } k=s(2 h 1 p), \\
\mathbf{N}_{\alpha u}^{(\mathrm{Ib})} & \text { if } k=u(3 h 2 p),\end{cases} \\
& \mathbf{C}_{j j^{\prime}}^{[\mathrm{ADC}(2)]}=0, \\
& \mathbf{D}_{k k^{\prime}}^{[\mathrm{ADC}(2)]}=0 .
\end{aligned}
$$

There are no interaction matrices $\mathbf{C}_{j j^{\prime}}$ and $\mathbf{D}_{k k^{\prime}}$ in the $\operatorname{ADC}(2)$, because coupling matrices are linked directly without any intermediate interaction insertion. This is not true anymore in the $\mathrm{ADC}(3)$, where matrices $\mathbf{M}^{\text {(Ia) }}$ and $\mathbf{N}^{(\mathrm{Ia})}$ are linked through interaction matrices $\mathbf{C}_{j j^{\prime}}$ and $\mathbf{D}_{k k^{\prime}}$ respectively, as it is the case for the third and sixth lines of Eq. (32). 


\section{B. ADC method at third order: $\mathrm{ADC}(3)$}

In this section we present explicit expressions of the coupling and interaction matrices entering in the ADC formalism at third order, for the three diagrams shown in Fig. 2 and for the four diagrams appearing first in each row of Fig. 6. The diagrams in Fig. 2 contribute to the block-diagonal entries $\left(r r^{\prime}\right)$ and $\left(s s^{\prime}\right)$ of Eq. 28, corresponding to $2 p 1 h$ and $2 h 1 p$ ISCs, which are the simplest configurations to be excited in the Fock space. The diagrams depicted in Figs. $2 \mathrm{a}$ and $2 \mathrm{~b}$ are the dominant ones at third order, given that only $2 \mathrm{~N}$ interactions are present. The diagram in Fig. 2c contains instead a $3 \mathrm{NF}$, but it can nonetheless play a significant role, because its Goldstone diagrams feature only $2 p 1 h$ and $2 h 1 p$ ISCs.

Each row in Fig. 6 collects a different topology of diagrams in terms of number of effective $2 \mathrm{NFs}$ and interaction-irreducible $3 \mathrm{NFs}$ entering the diagrams. In general, these diagrams are less important compared to the ones in Fig. 2, because they feature at least a $3 p 2 h$ or a $3 h 2 p$ ISC in all their Goldstone contributions. For forward-in-time (backward-in-time) diagrams, topologies in the first and second row of Fig. 6 couple $2 p 1 h(2 h 1 p)$ ISCs to $3 p 2 h(3 h 2 p)$ ISCs. They are linked by $\mathbf{C}_{r q^{\prime}}$ $\left(\mathbf{D}_{s u^{\prime}}\right)$ and $\mathbf{C}_{q r^{\prime}}\left(\mathbf{D}_{u s^{\prime}}\right)$, accounting for off-diagonal entries of Eq. 28. Within these two kinds of topologies, diagrams in the first row contain only one $3 \mathrm{NF}$, therefore they are expected to be more important than the ones in the second row, each featuring two 3NFs. Finally, the last two rows in Fig. 6 introduce the diagonal coupling between ISCs with five fermionic lines, $3 p 2 h$ for forward-in-time diagrams and $3 h 2 p$ for backward-in-time diagrams, corresponding respectively to entries $\left(q q^{\prime}\right)$ and $\left(u u^{\prime}\right)$ of Eq. 28. Again, there is a hierarchy between the two topologies, with those in the fourth row being less important due to the presence of three 3NFs.

The first four diagrams in each row are assumed as emblematic for each topology, and treated in the present section. The remaining coupling and interaction matrices originating from third-order diagrams are given in Appendix A.

Coupling and interaction matrices are fully antisymmetrized with respect to their particle and hole indexes. To show this explicitly, we introduce the following antisymmetrizer operators. Given a function depending on up to three particle or hole indexes, i.e. $f(i, j, h) \equiv$ $f\left(n_{i}, n_{j}, n_{h}\right)$ or $f(i, j, h) \equiv f\left(k_{i}, k_{j}, k_{h}\right)$, the antisymmetric permutation operator of a pair of indexes is introduced,

$$
\mathcal{A}_{i j} f(i, j, h) \equiv f(i, j, h)-f(j, i, h)
$$

For $3 p 2 h$ and $3 h 2 p$ configurations, it is useful to define the cyclic permutation operator as

$$
\mathcal{P}_{i j k} f(i, j, h) \equiv f(i, j, h)+f(h, i, j)+f(j, h, i),
$$

and the permutation operator acting on three indexes, that is

$$
\begin{aligned}
\mathcal{A}_{i j h} f(i, j, h) & \equiv f(i, j, h)+f(h, i, j)+f(j, h, i) \\
& -f(i, h, j)-f(j, i, h)-f(h, j, i) .
\end{aligned}
$$

Coupling matrices appearing at third order contain two interaction operators, which can be the interactionirreducible $3 \mathrm{NF}$ and/or the effective $2 \mathrm{NF}$. To simplify the equations, we write in compact form pieces of diagrams that correspond to the amplitudes appearing in the exponential ansatz of the coupled-cluster wave function 36. Without assuming the Einstein's convention of summing over repeated indices, we write them as,

$$
t_{k_{2}}^{n_{1}} \equiv \sum_{\alpha \beta} \frac{\mathcal{X}_{\alpha}^{n_{1}} \widetilde{U}_{\alpha \beta} \mathcal{Y}_{\beta}^{k_{2}}}{\varepsilon_{k_{2}}^{-}-\varepsilon_{n_{1}}^{+}}
$$

with the effective one-body potential of Eq. (13),

$$
t_{k_{3} k_{4}}^{n_{1} n_{2}} \equiv \sum_{\substack{\alpha \beta \\ \gamma \delta}} \frac{\mathcal{X}_{\alpha}^{n_{1}} \mathcal{X}_{\beta}^{n_{2}} \widetilde{V}_{\alpha \beta, \gamma \delta} \mathcal{Y}_{\gamma}^{k_{3}} \mathcal{Y}_{\delta}^{k_{4}}}{\varepsilon_{k_{3}}^{-}+\varepsilon_{k_{4}}^{-}-\varepsilon_{n_{1}}^{+}-\varepsilon_{n_{2}}^{+}}
$$

with the effective $2 \mathrm{NF}$ of Eq. (15), and

$$
t_{k_{4} k_{5} k_{6}}^{n_{1} n_{2} n_{3}} \equiv \sum_{\substack{\alpha \beta \gamma \\ \mu \nu \lambda}} \frac{\mathcal{X}_{\alpha}^{n_{1}} \mathcal{X}_{\beta}^{n_{2}} \mathcal{X}_{\gamma}^{n_{3}} W_{\alpha \beta \gamma, \mu \nu \lambda} \mathcal{Y}_{\mu}^{k_{4}} \mathcal{Y}_{\nu}^{k_{5}} \mathcal{Y}_{\lambda}^{k_{6}}}{\varepsilon_{k_{4}}^{-}+\varepsilon_{k_{5}}^{-}+\varepsilon_{k_{6}}^{-}-\varepsilon_{n_{1}}^{+}-\varepsilon_{n_{2}}^{+}-\varepsilon_{n_{3}}^{+}},
$$

for the terms with the interaction-irreducible $3 \mathrm{NF}$.

\section{ADC(3) matrices for Feynman diagrams in Fig. 2}

At third order in the ADC, we consider first the subset of coupling matrices and interaction matrices that are linked to $2 p 1 h$ and $2 h 1 p$ ISCs. For these intermediate configurations, the $\mathrm{ADC}(3)$ approximation for Eq. 19 requires the following terms,

$$
\begin{aligned}
& \mathbf{M}_{j \alpha}^{(\mathrm{II})}=\mathbf{M}_{r \alpha}^{(\mathrm{IIa})}+\mathbf{M}_{r \alpha}^{(\mathrm{IIb})}+\mathbf{M}_{r \alpha}^{(\mathrm{IIc})} \\
& \mathbf{N}_{\alpha k}^{(\mathrm{II})}=\mathbf{N}_{\alpha s}^{(\mathrm{IIa})}+\mathbf{N}_{\alpha s}^{(\mathrm{IIb})}+\mathbf{N}_{\alpha s}^{(\mathrm{IIc})} \\
& \mathbf{C}_{j j^{\prime}}=\mathbf{C}_{r r^{\prime}}^{p p}+\mathbf{C}_{r r^{\prime}}^{p h}+\mathbf{C}_{r r^{\prime}}^{3 N} \\
& \mathbf{D}_{k k^{\prime}}=\mathbf{D}_{s s^{\prime}}^{h h}+\mathbf{D}_{s s^{\prime}}^{h p}+\mathbf{D}_{s s^{\prime}}^{3 N}
\end{aligned}
$$

in addition to the ones already introduced by Eqs. 39 . 42 at second order.

We show now explicit expressions for the right-hand side of Eqs. (49)-(52), and start by presenting coupling matrices composed by two effective 2NFs connecting to $2 p 1 h$ ISCs. By using the definition in Eq. (47), we have the matrices,

$$
\mathbf{M}_{r \alpha}^{(\mathrm{IIa})} \equiv \frac{1}{2 \sqrt{2}} t_{k_{4} k_{5}}^{n_{1} n_{2}}\left(\mathcal{Y}_{\mu}^{k_{4}} \mathcal{Y}_{\nu}^{k_{5}}\right)^{*} \mathcal{Y}_{\lambda}^{k_{3}} \widetilde{V}_{\mu \nu, \alpha \lambda}
$$




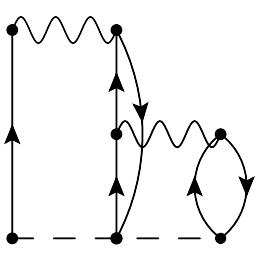

(d)

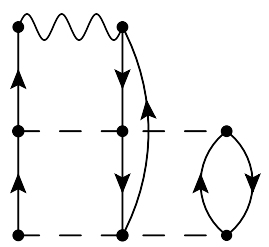

(h)

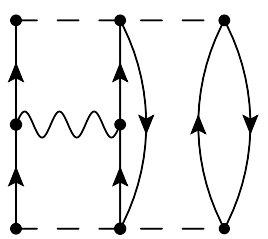

(1)

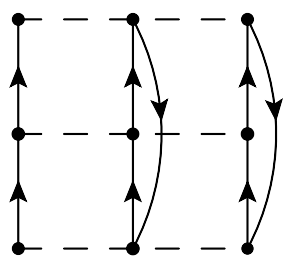

(o)

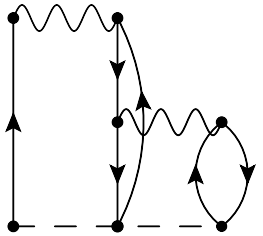

(e)

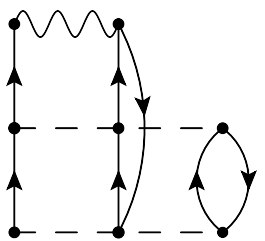

(i)

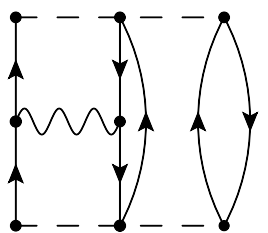

(m)

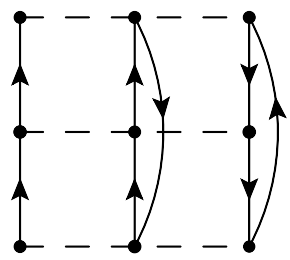

(p)

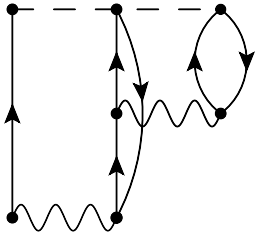

(f)

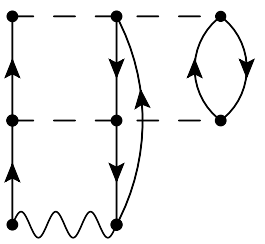

(j)

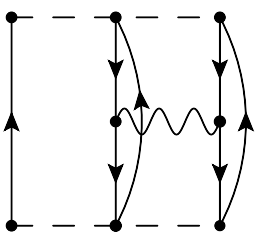

(n)

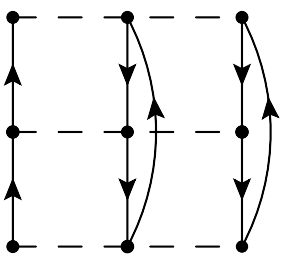

(q)

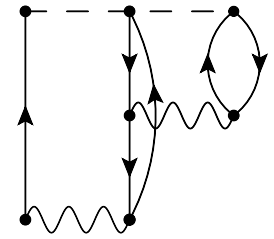

(g)

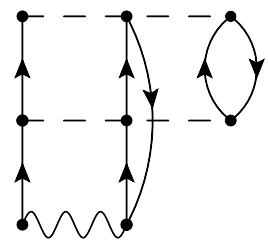

(k)

FIG. 6. As in Fig. 1 but for third-order diagrams that include any $3 p 2 h$ and $3 h 2 p$ intermediate state configurations. Together with the diagrams (a), (b) and (c) of Fig. 2, these are all skeleton and interaction-irreducible contributions present at third order. The labels in each diagram match the naming used in the text for of the corresponding coupling matrices.

and

$$
\begin{aligned}
\mathbf{M}_{r \alpha}^{(\mathrm{IIb})} \equiv & \frac{1}{\sqrt{2}}\left(t_{k_{3} k_{5}}^{n_{2} n_{4}} \mathcal{X}_{\mu}^{n_{1}}\left(\mathcal{Y}_{\nu}^{k_{5}} \mathcal{X}_{\lambda}^{n_{4}}\right)^{*} \widetilde{V}_{\mu \nu, \alpha \lambda}\right. \\
& \left.-t_{k_{3} k_{5}}^{n_{1} n_{4}} \mathcal{X}_{\mu}^{n_{2}}\left(\mathcal{Y}_{\nu}^{k_{5}} \mathcal{X}_{\lambda}^{n_{4}}\right)^{*} \widetilde{V}_{\mu \nu, \alpha \lambda}\right),
\end{aligned}
$$

which is explicitly antisymmetrized with respect to the $n_{1}, n_{2}$ fermion lines. The two coupling matrices in Eqs. (53) and (54) are found in the Goldstone diagrams of the terms in Figs. 2a and 2b, as it is clear from their diagrammatic representations in Figs. $7 \mathrm{a}$ and $7 \mathrm{~b}$ respectively.

The corresponding coupling matrices in the $2 h 1 p$ sector read,

$$
\mathbf{N}_{\alpha s}^{(\mathrm{IIa})} \equiv \frac{1}{2 \sqrt{2}} \widetilde{V}_{\alpha \lambda, \mu \nu} \mathcal{X}_{\lambda}^{n_{3}}\left(\mathcal{X}_{\mu}^{n_{4}} \mathcal{X}_{\nu}^{n_{5}}\right)^{*} t_{k_{1} k_{2}}^{n_{4} n_{5}},
$$

and

$$
\begin{aligned}
\mathbf{N}_{\alpha s}^{(\mathrm{IIb})} \equiv & \frac{1}{\sqrt{2}}\left(\widetilde{V}_{\alpha \lambda, \mu \nu} \mathcal{Y}_{\mu}^{k_{1}}\left(\mathcal{X}_{\nu}^{n_{4}} \mathcal{Y}_{\lambda}^{k_{5}}\right)^{*} t_{k_{5} k_{2}}^{n_{4} n_{3}}\right. \\
& \left.-\widetilde{V}_{\alpha \lambda, \mu \nu} \mathcal{Y}_{\mu}^{k_{2}}\left(\mathcal{X}_{\nu}^{n_{4}} \mathcal{Y}_{\lambda}^{k_{5}}\right)^{*} t_{k_{5} k_{1}}^{n_{4} n_{3}}\right) .
\end{aligned}
$$

Both matrices in Eqs. (55) and (56) are linked to $2 h 1 p$ ISCs, as it is clear by considering backward-in-time Goldstone terms of diagrams in Figs. 2a and 2b respectively. Their diagrammatic representations are displayed in Figs. 8a and 8b respectively. 


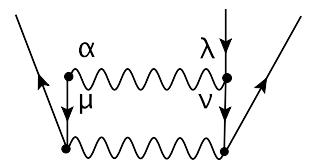

(a)

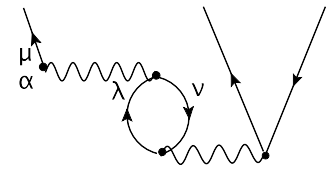

(b)

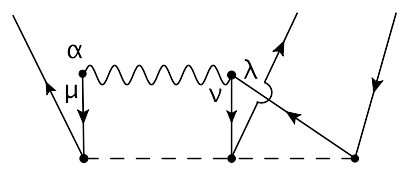

(c)

FIG. 7. Forward-in-time diagrams of the self-energy coupling matrices in $\operatorname{ADC}(3)$. Coupling matrices (a) and (b) correspond to Eqs. (53) and (54), and they feature two effective 2 NFs $\widetilde{V}$ connecting $2 p 1 h$ ISCs. The coupling matrix (c) contains one effective $2 \mathrm{NF} \widetilde{V}$ and one interaction-irreducible $3 \mathrm{NF} \hat{W}$. It connects to $2 p 1 h$ ISCs and corresponds to Eq. (57).

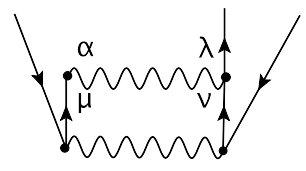

(a)

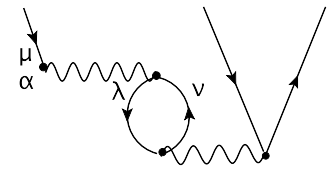

(b)

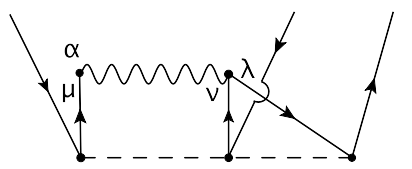

(c)

FIG. 8. Same as in Fig. 7 but for backward-in-time diagrams. Coupling matrices (a) and (b) correspond to Eqs. (55) and (56), while the coupling matrix (c) to Eq. (58).

Other coupling matrices containing two effective 2NFs can be found in Sec. A1 of Appendix A.

Among coupling matrices containing one effective $2 \mathrm{NF}$ and one interaction-irreducible $3 \mathrm{NF}$, we present here the ones appearing in the self-energy diagram of Fig. 20, that is

$$
\mathbf{M}_{r \alpha}^{(\mathrm{IIc})} \equiv \frac{1}{2 \sqrt{2}} t_{k_{5} k_{6} k_{3}}^{n_{1} n_{2} n_{4}}\left(\mathcal{Y}_{\mu}^{k_{5}} \mathcal{Y}_{\nu}^{k_{6}} \mathcal{X}_{\lambda}^{n_{4}}\right)^{*} \widetilde{V}_{\mu \nu, \alpha \lambda},
$$

and

$$
\mathbf{N}_{\alpha s}^{(\mathrm{IIc})} \equiv-\frac{1}{2 \sqrt{2}} \widetilde{V}_{\alpha \lambda, \mu \nu}\left(\mathcal{X}_{\mu}^{n_{5}} \mathcal{X}_{\nu}^{n_{6}} \mathcal{Y}_{\lambda}^{k_{4}}\right)^{*} t_{k_{1} k_{2} k_{4}}^{n_{5} n_{6} n_{3}}
$$

Diagrammatic representations of Eqs. (57) and (58) are displayed in Figs. $7 \mathrm{c}$ and $8 \mathrm{c}$ respectively.
All the other coupling matrices with one $2 \mathrm{NF}$ and one $3 \mathrm{NF}$ are collected in Sec. A 2 of Appendix A while matrices with two interaction-irreducible $3 \mathrm{NFs}$ are presented in Sec. A3 in Appendix A.

Now we introduce expressions of the interaction matrices of Eqs. 51 and 52 , containing the $\widetilde{V}_{\alpha \beta, \gamma \delta}$ and $W_{\alpha \beta \gamma, \delta \epsilon \lambda}$ matrix elements. For the two cases, we display interaction matrices appearing in both the forwardin-time and backward-in-time self-energy Goldstone diagrams of Fig. 2 .

The interaction matrix that connects $2 p 1 h$ ISCs through a particle-particle $(p p)$ interaction is

$$
\mathbf{C}_{r r^{\prime}}^{p p} \equiv \frac{1}{2} \mathcal{X}_{\mu}^{n_{1}} \mathcal{X}_{\nu}^{n_{2}} \widetilde{V}_{\mu \nu, \lambda \rho}\left(\mathcal{X}_{\lambda}^{n_{4}} \mathcal{X}_{\rho}^{n_{5}}\right)^{*} \delta_{k_{3} k_{6}},
$$

while the one connecting through a particle-hole $(p h)$ interaction is

$$
\mathbf{C}_{r r^{\prime}}^{p h} \equiv \frac{1}{2} \mathcal{A}_{12} \mathcal{A}_{45}\left(\mathcal{X}_{\nu}^{n_{1}} \mathcal{Y}_{\rho}^{k_{3}} \widetilde{V}_{\nu \mu, \rho \lambda}\left(\mathcal{X}_{\lambda}^{n_{4}} \mathcal{Y}_{\mu}^{k_{6}}\right)^{*} \delta_{n_{2} n_{5}}\right),
$$

where the action of two permutation operators $\mathcal{A}_{12}$ and $\mathcal{A}_{45}$ is defined in Eq. 43 and produces four terms.

We present now the corresponding interaction matrices appearing in backward-in-time self-energy Goldstone diagrams, namely those that are linked to propagators of hole-particle kind in diagrams.

We start by the interaction matrix that connects $2 h 1 p$ ISCs through a hole-hole $(h h)$ interaction, that is

$$
\mathbf{D}_{s s^{\prime}}^{h h} \equiv-\frac{1}{2}\left(\mathcal{Y}_{\mu}^{k_{1}} \mathcal{Y}_{\nu}^{k_{2}}\right)^{*} \widetilde{V}_{\mu \nu, \lambda \rho} \mathcal{Y}_{\lambda}^{k_{4}} \mathcal{Y}_{\rho}^{k_{5}} \delta_{n_{3} n_{6}},
$$

while the one connecting through a hole-particle $(h p)$ interaction is

$$
\mathbf{D}_{s s^{\prime}}^{h p} \equiv-\frac{1}{2} \mathcal{A}_{12} \mathcal{A}_{45}\left(\left(\mathcal{Y}_{\mu}^{k_{1}} \mathcal{X}_{\rho}^{n_{3}}\right)^{*} \widetilde{V}_{\mu \nu, \rho \lambda} \mathcal{Y}_{\lambda}^{k_{4}} \mathcal{X}_{\nu}^{n_{6}} \delta_{k_{2} k_{5}}\right),
$$

where permutation operators acting on hole states are defined in the same way as the one acting on particle states for the matrix $\mathbf{C}_{r r^{\prime}}^{p h}$ in Eq. 60).

The only interaction matrix that connects $2 p 1 h$ ISCs through a $3 \mathrm{NF}$ is

$$
\mathbf{C}_{r r^{\prime}}^{3 N} \equiv-\frac{1}{2} \mathcal{X}_{\nu}^{n_{1}} \mathcal{X}_{\mu}^{n_{2}} \mathcal{Y}_{\rho}^{k_{3}} W_{\nu \mu \lambda, \epsilon \eta \rho}\left(\mathcal{X}_{\epsilon}^{n_{4}} \mathcal{X}_{\eta}^{n_{5}} \mathcal{Y}_{\lambda}^{k_{6}}\right)^{*},
$$

which is explicitly antisymmetric in particle indexes, while the one connecting two $2 h 1 p$ ISCs through a $3 \mathrm{NF}$ is

$$
\mathbf{D}_{s s^{\prime}}^{3 N} \equiv-\frac{1}{2}\left(\mathcal{Y}_{\nu}^{k_{1}} \mathcal{Y}_{\mu}^{k_{2}} \mathcal{X}_{\rho}^{n_{3}}\right)^{*} W_{\nu \mu \lambda, \epsilon \eta \rho} \mathcal{Y}_{\epsilon}^{k_{4}} \mathcal{Y}_{\eta}^{k_{5}} \mathcal{X}_{\lambda}^{n_{6}}
$$

which is also explicitly antisymmetric in hole indexes.

\section{2. $A D C(3)$ matrices for selected}




\section{Feynman diagrams in Fig. 6 .}

Coupling matrices presented in this section are obtained from the four Feynman diagrams in the first column of Fig. 6. Most of these matrices are linked to $3 p 2 h$ and $3 h 2 p$ ISCs, with few exceptions derived from Goldstone diagrams where ISCs are of $2 p 1 h$ and $2 h 1 p$ type. This subset of coupling matrices at the $\mathrm{ADC}(3)$ level is given by

$$
\begin{aligned}
& \mathbf{M}_{j \alpha}^{(\mathrm{II})}: \mathbf{M}_{q \alpha}^{(\mathrm{IId})} ; \mathbf{M}_{r \alpha}^{\left(\mathrm{IId}^{\prime}\right)} ; \mathbf{M}_{q \alpha}^{(\mathrm{IIh})} ; \mathbf{M}_{r \alpha}^{\left(\mathrm{IIh}^{\prime}\right)} ; \mathbf{M}_{q \alpha}^{(\mathrm{IIl})} ; \mathbf{M}_{q \alpha}^{(\mathrm{IIo})} \\
& \mathbf{N}_{\alpha k}^{(\mathrm{II})}: \mathbf{N}_{\alpha u}^{(\mathrm{IId})} ; \mathbf{N}_{\alpha s}^{\left(\mathrm{IId}^{\prime}\right)} ; \mathbf{N}_{\alpha u}^{(\mathrm{IIh})} ; \mathbf{N}_{\alpha s}^{\left(\mathrm{IIh}^{\prime}\right)} ; \mathbf{N}_{\alpha u}^{(\mathrm{IIl})} ; \mathbf{N}_{\alpha u}^{(\mathrm{IIo})} \cdot(66)
\end{aligned}
$$

First, we present matrices containing two effective 2NFs. As before, we display both matrices obtained from forward-in-time and backward-in-time Goldstone diagrams, denoted with the notation $\mathbf{M}_{j \alpha}$ and $\mathbf{N}_{\alpha k}$ respectively.

In Goldstone diagrams of the term in Fig. 6d we have,

$$
\mathbf{M}_{q \alpha}^{(\mathrm{IId})} \equiv \frac{\sqrt{3}}{6} \mathcal{A}_{45} \mathcal{P}_{123}\left(t_{k_{5} k_{6}}^{n_{1} n_{2}} \mathcal{X}_{\mu}^{n_{3}}\left(\mathcal{Y}_{\nu}^{k_{6}}\right)^{*} \mathcal{Y}_{\lambda}^{k_{4}} \widetilde{V}_{\mu \nu, \alpha \lambda}\right)
$$

where the combination of permutation operators performs the antisymmetrization of the indexes $\left(k_{4}, k_{5}\right)$, $\left(n_{1}, n_{3}\right)$ and $\left(n_{2}, n_{3}\right)$, according to definitions in Eqs. (43) and 44.

We turn now to coupling matrices containing one effective $2 \mathrm{NF}$ and one interaction-irreducible $3 \mathrm{NF}$. In the Goldstone diagrams of the term in Fig. 6d we have also,

$$
\begin{aligned}
\mathbf{M}_{r \alpha}^{\left(\mathrm{IId}^{\prime}\right)} \equiv & -\frac{\sqrt{2}}{4} \mathcal{A}_{12}\left(\mathcal{X}_{\lambda}^{n_{1}} t_{k_{5} k_{6}}^{n_{2} n_{4}}\left(\mathcal{Y}_{\mu}^{k_{5}} \mathcal{Y}_{\nu}^{k_{6}} \mathcal{X}_{\rho}^{n_{4}}\right)^{*} \mathcal{Y}_{\eta}^{k_{3}}\right. \\
& \left.W_{\lambda \mu \nu, \alpha \eta \rho}\right) .
\end{aligned}
$$

In the Goldstone diagrams of the term in Fig. 6h we have,

$$
\mathbf{M}_{q \alpha}^{(\mathrm{IIh})} \equiv \frac{\sqrt{3}}{6} \mathcal{P}_{123}\left(t_{k_{4} k_{5} k_{7}}^{n_{1} n_{3} n_{6}}\left(\mathcal{Y}_{\mu}^{k_{7}}\right)^{*} \mathcal{X}_{\nu}^{n_{2}}\left(\mathcal{X}_{\lambda}^{n_{6}}\right)^{*} \widetilde{V}_{\mu \nu, \alpha \lambda}\right)
$$

while in the Goldstone diagrams of the term in Fig. 61 we find,

$\mathbf{M}_{q \alpha}^{(\mathrm{IIl})} \equiv \frac{\sqrt{3}}{12} \mathcal{P}_{123}\left(t_{k_{6} k_{7}}^{n_{1} n_{2}}\left(\mathcal{Y}_{\mu}^{k_{6}} \mathcal{Y}_{\nu}^{k_{7}}\right)^{*} \mathcal{X}_{\lambda}^{n_{3}} \mathcal{Y}_{\eta}^{k_{4}} \mathcal{Y}_{\rho}^{k_{5}} W_{\mu \nu \lambda, \alpha \eta \rho}\right)$

For backward-in-time Goldstone diagrams, we can single out from the term in Fig. 6d the coupling matrix,

$$
\mathbf{N}_{\alpha u}^{(\mathrm{IId})} \equiv \frac{\sqrt{3}}{6} \widetilde{V}_{\alpha \lambda, \mu \nu} \mathcal{A}_{45} \mathcal{P}_{123}\left(\mathcal{Y}_{\mu}^{k_{1}}\left(\mathcal{X}_{\nu}^{n_{6}}\right)^{*} \mathcal{X}_{\lambda}^{n_{4}} t_{k_{2} k_{3}}^{n_{6} n_{5}}\right)
$$

and also the following coupling matrix depending on $2 \mathrm{~N}$ and $3 \mathrm{~N}$ interactions, i.e.

$$
\begin{aligned}
\mathbf{N}_{\alpha s}^{\left(\mathrm{IId}^{\prime}\right)} \equiv & \frac{\sqrt{2}}{4} W_{\alpha \eta \rho, \lambda \mu \nu} \mathcal{A}_{12}\left(\mathcal{X}_{\eta}^{n_{3}}\left(\mathcal{Y}_{\rho}^{k_{4}}\right)^{*} \mathcal{Y}_{\lambda}^{k_{1}}\left(\mathcal{X}_{\mu}^{n_{5}} \mathcal{X}_{\nu}^{n_{6}}\right)^{*}\right. \\
& \left.t_{k_{2} k_{4}}^{n_{5} n_{6}}\right) .
\end{aligned}
$$

In Fig. 6h, we have the backward-in-time the coupling matrix,

$$
\mathbf{N}_{\alpha u}^{(\mathrm{IIh})} \equiv \frac{\sqrt{3}}{6} \widetilde{V}_{\alpha \lambda, \mu \nu} \mathcal{P}_{123}\left(\left(\mathcal{Y}_{\lambda}^{k_{7}} \mathcal{X}_{\mu}^{n_{6}}\right)^{*} \mathcal{Y}_{\nu}^{k_{2}} t_{k_{1} k_{3} k_{7}}^{n_{6} n_{4} n_{5}}\right)
$$

while in Fig. 61 we find the coupling matrix,

$$
\begin{aligned}
\mathbf{N}_{\alpha u}^{(\mathrm{III})} \equiv & \frac{\sqrt{3}}{12} W_{\alpha \eta \rho, \mu \nu \lambda} \mathcal{P}_{123}\left(\mathcal{X}_{\eta}^{n_{4}} \mathcal{X}_{\rho}^{n_{5}}\left(\mathcal{X}_{\mu}^{n_{6}} \mathcal{X}_{\nu}^{n_{7}}\right)^{*} \mathcal{Y}_{\lambda}^{k_{3}}\right. \\
& \left.t_{k_{1} k_{2}}^{n_{6} n_{7}}\right) .
\end{aligned}
$$

Finally we introduce coupling matrices containing two interaction-irreducible 3NFs. In Goldstone diagrams of the term in Fig. 6h we have,

$$
\begin{aligned}
\mathbf{M}_{r \alpha}^{\left(\mathrm{IIh}^{\prime}\right)} \equiv & \frac{\sqrt{2}}{8} \mathcal{A}_{12}\left(t_{k_{6} k_{3} k_{7}}^{n_{1} n_{4} n_{5}}\left(\mathcal{Y}_{\mu}^{k_{6}} \mathcal{Y}_{\nu}^{k_{7}}\right)^{*} \mathcal{X}_{\lambda}^{n_{2}}\left(\mathcal{X}_{\eta}^{n_{4}} \mathcal{X}_{\rho}^{n_{5}}\right)^{*}\right. \\
& \left.W_{\lambda \mu \nu, \alpha \eta \rho}\right)
\end{aligned}
$$

while in Goldstone diagrams of the term in Fig. 6o we have,

$\mathbf{M}_{q \alpha}^{(\mathrm{IIo})} \equiv-\frac{\sqrt{3}}{36} t_{k_{6} k_{7} k_{8}}^{n_{1} n_{2} n_{3}}\left(\mathcal{Y}_{\mu}^{k_{6}} \mathcal{Y}_{\nu}^{k_{7}} \mathcal{Y}_{\lambda}^{k_{8}}\right)^{*} \mathcal{Y}_{\eta}^{k_{4}} \mathcal{Y}_{\rho}^{k_{5}} W_{\mu \nu \lambda, \alpha \eta \rho}$

that is antisymmetric in the indexes $n_{1}, n_{2}, n_{3}$ and $k_{4}, k_{5}$.

For backward-in-time Goldstone diagrams, we can single out from the term in Fig. 6h the coupling matrix

$$
\begin{aligned}
\mathbf{N}_{\alpha s}^{\left(\mathrm{IIh}^{\prime}\right)} \equiv & \frac{\sqrt{2}}{8} W_{\alpha \eta \rho, \mu \lambda \nu} \mathcal{A}_{12}\left(\left(\mathcal{Y}_{\eta}^{k_{4}} \mathcal{Y}_{\rho}^{k_{5}} \mathcal{X}_{\mu}^{n_{6}} \mathcal{X}_{\nu}^{n_{7}}\right)^{*} \mathcal{Y}_{\lambda}^{k_{2}}\right. \\
& \left.t_{k_{1} k_{4} k_{5}}^{n_{3} n_{6} n_{7}}\right)
\end{aligned}
$$

while the matrix

$$
\mathbf{N}_{\alpha u}^{(\mathrm{IIo})} \equiv \frac{\sqrt{3}}{36} W_{\alpha \eta \rho, \mu \nu \lambda} \mathcal{X}_{\eta}^{n_{4}} \mathcal{X}_{\rho}^{n_{5}}\left(\mathcal{X}_{\mu}^{n_{6}} \mathcal{X}_{\nu}^{n_{7}} \mathcal{X}_{\lambda}^{n_{8}}\right)^{*} t_{k_{1} k_{2} k_{3}}^{n_{6} n_{7} n_{8}}
$$

appears in the Goldstone diagrams relative to Fig. 60 and it is antisymmetric in the indexes $k_{1}, k_{2}, k_{3}$ and $n_{4}, n_{5}$.

\section{CONCLUSIONS}

We have calculated all possible Feynman diagrams for the self-energy up to the third order, for an Hamiltonian including up to three-body interactions. Using these, 
we have then derived the complete set of working equations that are needed to calculate the self-energy nonperturbatively in the $\operatorname{ADC}(n)$ approach at orders $n=2$ and 3. While the expansion of the self-energy is considered perturbatively by including diagrams featuring up to three interactions, the $\mathrm{ADC}(3)$ formalism expands automatically certain classes of diagrams to infinite order. In particular, one resums series of ladders, rings and interaction-irreducible 3NFs diagrams. As for the usual $\operatorname{ADC}(n)$ computations, the Dyson equation for the $1 \mathrm{~B}$ propagator can be implemented as a large but energy independent eigenvalue problem. However, in presence of $3 \mathrm{NFs}$, intermediate state configurations of $3 p 2 h$ and $3 h 2 p$ type contribute already at $\mathrm{ADC}(2)$ and $\mathrm{ADC}(3)$ levels, while they would appear at $\operatorname{ADC}(4)$ and $\operatorname{ADC}(5)$ for NN-only interactions.

In showing expressions for both coupling and interaction matrices, we have organized the equations according to their importance, using criteria based on the number of excitations implied by ISCs and the natural hierarchy of many-nucleon forces. We started by revisiting the most relevant correlations in terms of $2 p 1 h$ and $2 h 1 p$ ISCs. This sector contains the well-known ADC(3) equations for the original and effective $\mathrm{NN}$ interactions. A new contribution arises from the Feynman diagram of Fig. 2c and involves an interaction-irreducible 3NF (that is, which cannot be expressed as simpler normal ordered forces). This last term is argued to be less relevant in virtue of the hierarchy of nuclear forces. Then, we have worked out the subset of $\mathrm{ADC}(3)$ coupling and interaction matrices that link to the $3 p 2 h$ and $3 h 2 p$ sector of ISCs. While this hierarchy suggests that $3 p 2 h$ and $3 h 2 p$ ISCs may be necessary only for future generations of $a b$ initio approaches, the diagram of Fig. 2c may already have implication for present nuclear Hamiltonians. However, these conjectures have not yet been checked and knowing the importance of diagram 2c would give guidance for the inclusion of further correlations [37.

To provide the ADC formalism in its most general form, we have released the assumption of a fully selfconsistent expansion and considered also all the additional nonskeleton diagrams that appear in this case. The resulting corrections are important (at least conceptually) when calculations are based on standard reference propagators of mean-field type. New sets of diagrams appear for both the static and dynamic self-energy and have been derived together with the corresponding contributions in the ADC framework. In total, four additional Feynman diagrams must be considered in the $\mathrm{ADC}(3)$ dynamic self-energy when one is working with uncorrelated propagators, while the $1 \mathrm{~B}$ effective interaction defining the energy-independent self-energy is decomposed into 19 Feynman diagrams of different topologies. Hence, the complete $\mathrm{ADC}(3)$ formalism with $3 \mathrm{NFs}$ is now available for the self-energy, either self-consistent (with only skeleton diagrams) or based on an uncorrelated reference state.

The formalism presented in this work sets the basis for future advancements of the SCGF approach, especially useful for studies of nuclear structure where the full inclusion of realistic three-nucleon interactions is required. The numerical implementation of the $3 p 2 h$ and $3 h 2 p$ sector is a long-term endeavour that might rely on future supercomputing computing resources. At the same time, the case for such improvements in the many-body truncation will also depend on the performance and accuracy of future generations of realistic nuclear interactions. On the other hand, calculating the diagram of Fig. 2c involves only $2 p 1 h$ and $2 h 1 p$ ISCs and will not require resources beyond present day computer power [38]. Thus, we plan a follow-up study to investigate this term.

\section{ACKNOWLEDGMENTS}

This work was supported by the United Kingdom Science and Technology Facilities Council (STFC), through Grants No. ST/L005743/1 and No. ST/P005314/1.

Appendix A: ADC equations for self-energy at third order

In this Appendix we give the remaining expressions of the coupling and interaction matrices that arise from Goldstone diagrams at third order in the expansion of the self-energy. The complete list of all terms required to build the 
$\mathrm{ADC}(3)$ formalism is

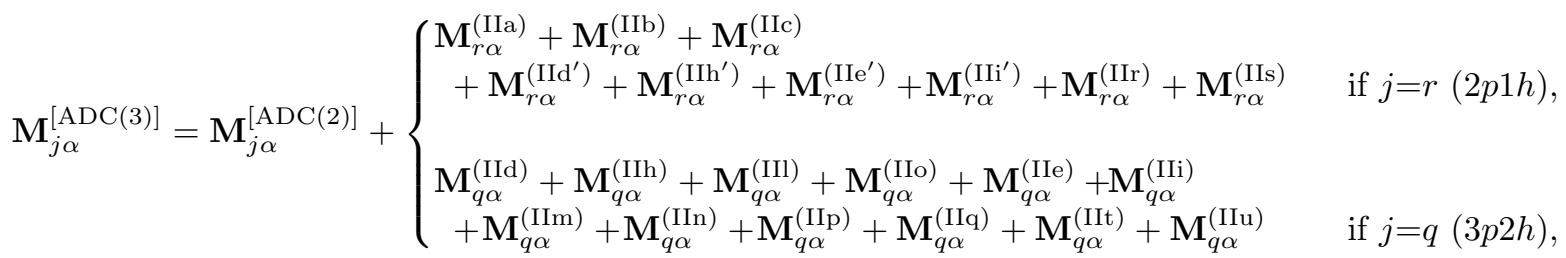

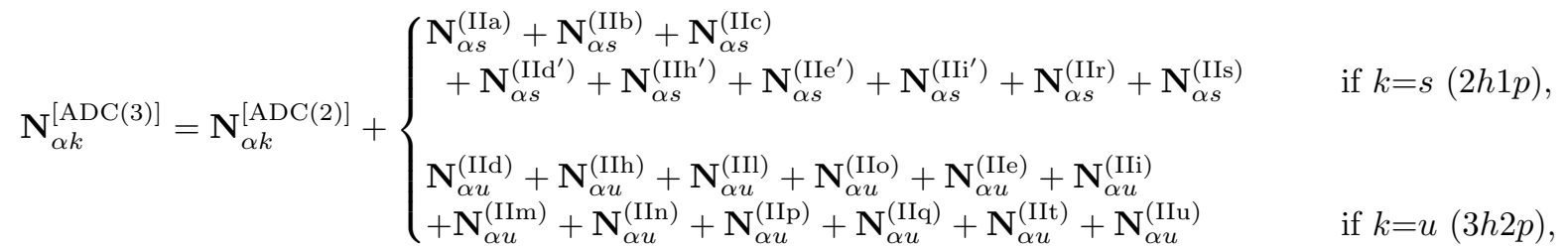

$$
\begin{aligned}
& \left(\mathbf{C}_{r r^{\prime}}^{p p}+\mathbf{C}_{r r^{\prime}}^{p h}+\mathbf{C}_{r r^{\prime}}^{3 N}+\mathbf{C}_{r r^{\prime}}^{\widetilde{U} p}+\mathbf{C}_{r r^{\prime}}^{\widetilde{U} h} \quad \text { if } j=r \text { and } j^{\prime}=r^{\prime}(2 p 1 h),\right.
\end{aligned}
$$

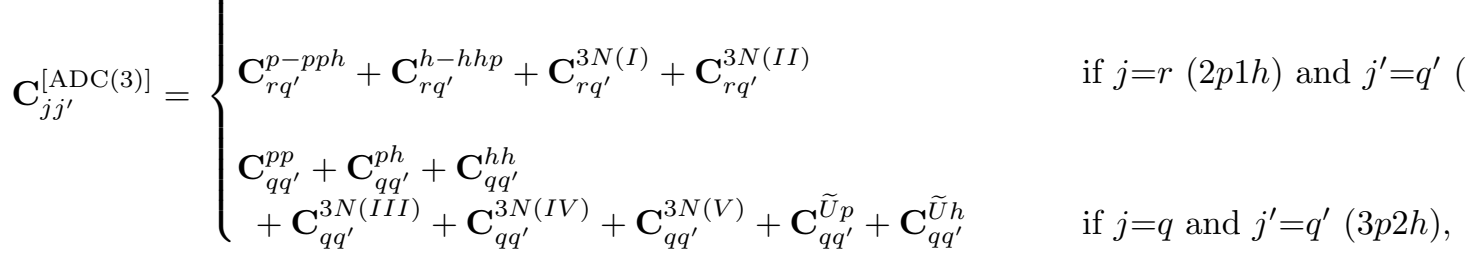

$$
\begin{aligned}
& \mathbf{D}_{k k^{\prime}}^{[\mathrm{ADC}(3)]}= \begin{cases}\mathbf{D}_{s s^{\prime}}^{h h}+\mathbf{D}_{s s^{\prime}}^{h p}+\mathbf{D}_{s s^{\prime}}^{3 N}+\mathbf{D}_{s s^{\prime}}^{\widetilde{U} p}+\mathbf{D}_{s s^{\prime}}^{\widetilde{U} h} & \text { if } k=s \text { and } k^{\prime}=s^{\prime}(2 h 1 p), \\
\mathbf{D}_{s u^{\prime}}^{h-h h p}+\mathbf{D}_{s u^{\prime}}^{p-p p h}+\mathbf{D}_{s u^{\prime}}^{3 N(I)}+\mathbf{D}_{s u^{\prime}}^{3 N(I I)} & \text { if } k=s(2 h 1 p) \text { and } k^{\prime}=u^{\prime}(3 h 2 p), \\
\mathbf{D}_{u u^{\prime}}^{h h}+\mathbf{D}_{u u^{\prime}}^{h p}+\mathbf{D}_{u u^{\prime}}^{p p} & \\
+\mathbf{D}_{u u^{\prime}}^{3 N(I I)}+\mathbf{D}_{u u^{\prime}}^{3 N(I V)}+\mathbf{D}_{u u^{\prime}}^{3 N(V)}+\mathbf{D}_{u u^{\prime}}^{\widetilde{U} p}+\mathbf{D}_{u u^{\prime}}^{\widetilde{U} h} & \text { if } k=u \text { and } k^{\prime}=u^{\prime}(3 h 2 p) .\end{cases}
\end{aligned}
$$

For the coupling matrices $\mathbf{M}_{j \alpha}$ and $\mathbf{N}_{\alpha k}$, the list of terms truncated at the $\mathrm{ADC}(3)$ level is composed by sets of $\mathrm{ADC}(2)$ terms, defined in Eqs. (34) and (37) and in Eqs. (35) and (38) for the forward-in-time and backwardin-time self-energy, respectively; sets of terms from (IIa) to (IIc) appearing at third order of the ADC, presented in Eqs. (53), (54), and (57) and in Eqs. (55)), (56), and (58), which contain only $2 p 1 h$ and $2 h 1 p$ configurations; and those terms from (IId) to (IIo) with $3 p 2 h$ and $3 h 2 p$ ISCs, introduced in Eqs. (67)-(70), (75), and (76) and in Eqs. (71)-(74), (77), and (78). Other terms with $3 p 2 h$ and $3 h 2 p$ ISCs, denoted with superscripts from (IIe) to (IIq), are defined in Eqs. A5)-A20. Moreover, in Eqs. A1 and A2 we find additional terms, that must be added to the $\mathrm{ADC}(3)$ when the single-particle propagator used to construct self-energy diagrams is uncorrelated, i.e. when one works with a nonskeleton expansion. For coupling matrices, these additional terms are denoted with superscripts ranging from (IIr) to (IIu). Their explicit expressions will be given in Appendix C2.

Interaction matrices appear at third order in the
$\mathrm{ADC}$, as listed in Eqs. $\mathrm{A} 3$ and $\mathrm{A} 4$. The first three terms thereof connecting to $2 p 1 h$ and $2 h 1 p$ configurations, are given in Eqs. (59), (60), and (63) for forwardin-time diagrams and in Eqs. (61), (62), and (64) for backward-in-time ones. Other matrices required to link $3 p 2 h(3 h 2 p)$ ISCs are denoted by $\mathbf{C}_{r q^{\prime}}^{p-p h}, \ldots, \mathbf{C}_{q q^{\prime}}^{3 N(V)}$ $\left(\mathbf{D}_{s u^{\prime}}^{h-h h}, \ldots, \mathbf{D}_{u u^{\prime}}^{3 N(V)}\right)$. They will be given below in Eqs. A21)-A25 and A31-A35 [Eqs. A26- A30 and $(\bar{A} 36)-(\bar{A} 40]$. Finally, additional four interaction matrices introduced in Appendix $\mathrm{C} 2$ for the nonskeleton expansion are specified in Eqs. A3 and A4 with the superscript $\widetilde{U}$.

\section{Coupling matrices with two effective 2NFs}

In Fig. 6e we find the following coupling matrices,

$$
\mathbf{M}_{q \alpha}^{(\mathrm{IIe})} \equiv \frac{\sqrt{3}}{6} \mathcal{P}_{123}\left(t_{k_{4} k_{5}}^{n_{6} n_{3}} \mathcal{X}_{\mu}^{n_{1}} \mathcal{X}_{\nu}^{n_{2}}\left(\mathcal{X}_{\lambda}^{n_{6}}\right)^{*} \widetilde{V}_{\mu \nu, \alpha \lambda}\right)
$$


and

$$
\mathbf{N}_{\alpha u}^{(\mathrm{IIe})} \equiv \frac{\sqrt{3}}{6} \widetilde{V}_{\alpha \lambda, \mu \nu} \mathcal{P}_{123}\left(\left(\mathcal{Y}_{\lambda}^{k_{6}}\right)^{*} \mathcal{Y}_{\mu}^{k_{1}} \mathcal{Y}_{\nu}^{k_{2}} t_{k_{6} k_{3}}^{n_{4} n_{5}}\right),
$$

for the forward-in-time and backward-in-time Goldstone diagrams, respectively.

\section{Coupling matrices with one effective $2 \mathrm{NF}$ and one interaction-irreducible $3 \mathrm{NF}$}

Diagrams in Fig. 6e contains also an interactionirreducible $3 \mathrm{NF}$, therefore another coupling matrix can be obtained from the corresponding Goldstone diagrams. For the forward-in-time and backward-in-time parts we have,

$$
\mathbf{M}_{r \alpha}^{\left(\mathrm{IIe}^{\prime}\right)} \equiv \frac{\sqrt{2}}{4} t_{k_{3} k_{6}}^{n_{4} n_{5}} \mathcal{X}_{\mu}^{n_{1}} \mathcal{X}_{\nu}^{n_{2}}\left(\mathcal{Y}_{\lambda}^{k_{6}} \mathcal{X}_{\rho}^{n_{4}} \mathcal{X}_{\eta}^{n_{5}}\right)^{*} W_{\mu \nu \lambda, \alpha \rho \eta}
$$

and

$$
\mathbf{N}_{\alpha s}^{\left(\mathrm{IIe}^{\prime}\right)} \equiv \frac{\sqrt{2}}{4} W_{\alpha \rho \eta, \mu \nu \lambda}\left(\mathcal{Y}_{\rho}^{k_{4}} \mathcal{Y}_{\eta}^{k_{5}}\right)^{*} \mathcal{Y}_{\mu}^{k_{1}} \mathcal{Y}_{\nu}^{k_{2}}\left(\mathcal{X}_{\lambda}^{n_{6}}\right)^{*} t_{k_{4} k_{5}}^{n_{3} n_{6}}
$$

respectively.

Also diagrams in the second and third row of Fig. 6 feature coupling matrices with $2 \mathrm{NFs}$ and interactionirreducible 3NFs. We list them below considering both forward- and backward-in-time contributions. In the Goldstone diagrams of the term in Fig. 6i we have,

$$
\mathbf{M}_{q \alpha}^{(\mathrm{IIi})} \equiv \frac{\sqrt{3}}{12} \mathcal{A}_{45}\left(t_{k_{5} k_{6} k_{7}}^{n_{1} n_{2} n_{3}}\left(\mathcal{Y}_{\mu}^{k_{6}} \mathcal{Y}_{\nu}^{k_{7}}\right)^{*} \mathcal{Y}_{\lambda}^{k_{4}} \widetilde{V}_{\mu \nu, \alpha \lambda}\right)
$$

and

$$
\mathbf{N}_{\alpha u}^{(\mathrm{IIi})} \equiv \frac{\sqrt{3}}{12} \mathcal{A}_{45}\left(\widetilde{V}_{\alpha \lambda, \mu \nu} \mathcal{X}_{\lambda}^{n_{4}}\left(\mathcal{X}_{\mu}^{n_{6}} \mathcal{X}_{\nu}^{n_{7}}\right)^{*} t_{k_{1} k_{2} k_{3}}^{n_{5} n_{6} n_{7}}\right)
$$

In the Goldstone diagrams of the term in Fig. $6 \mathrm{~m}$ we can single out the coupling matrices,

$$
\begin{aligned}
\mathbf{M}_{q \alpha}^{(\mathrm{IIm})} \equiv & \frac{\sqrt{3}}{6} \mathcal{A}_{45} \mathcal{P}_{123}\left(t_{k_{4} k_{7}}^{n_{1} n_{6}}\left(\mathcal{Y}_{\mu}^{k_{7}}\right)^{*} \mathcal{X}_{\nu}^{n_{2}} \mathcal{X}_{\lambda}^{n_{3}}\right. \\
& \left.\mathcal{Y}_{\eta}^{k_{5}}\left(\mathcal{X}_{\rho}^{n_{6}}\right)^{*} W_{\mu \nu \lambda, \alpha \rho \eta}\right)
\end{aligned}
$$

and

$$
\begin{aligned}
\mathbf{N}_{\alpha u}^{(\mathrm{IIm})} \equiv & \frac{\sqrt{3}}{6} \mathcal{A}_{45} \mathcal{P}_{123}\left(W_{\alpha \eta \rho, \mu \nu \lambda}\left(\mathcal{Y}_{\eta}^{k_{7}}\right)^{*} \mathcal{X}_{\rho}^{n_{5}}\right. \\
& \left.\left(\mathcal{X}_{\mu}^{n_{6}}\right)^{*} \mathcal{Y}_{\nu}^{k_{2}} \mathcal{Y}_{\lambda}^{k_{3}} t_{k_{1} k_{7}}^{n_{4} n_{6}}\right) .
\end{aligned}
$$

Finally, in the Goldstone diagrams of the term in Fig. 6n we have,

$$
\mathbf{M}_{q \alpha}^{(\mathrm{IIn})} \equiv \frac{\sqrt{3}}{12} t_{k_{4} k_{5}}^{n_{6} n_{7}} \mathcal{X}_{\mu}^{n_{1}} \mathcal{X}_{\nu}^{n_{2}} \mathcal{X}_{\lambda}^{n_{3}}\left(\mathcal{X}_{\eta}^{n_{6}} \mathcal{X}_{\rho}^{n_{7}}\right)^{*} W_{\mu \nu \lambda, \alpha \eta \rho}
$$

and

$$
\mathbf{N}_{\alpha u}^{(\mathrm{IIn})} \equiv \frac{\sqrt{3}}{12} W_{\alpha \eta \rho, \mu \nu \lambda} \mathcal{Y}_{\mu}^{k_{1}} \mathcal{Y}_{\nu}^{k_{2}} \mathcal{Y}_{\lambda}^{k_{3}}\left(\mathcal{Y}_{\eta}^{k_{6}} \mathcal{Y}_{\rho}^{k_{7}}\right)^{*} t_{k_{6} k_{7}}^{n_{4} n_{5}}
$$

that are both antisymmetric in their particle and hole indexes.

\section{Coupling matrices with two interaction-irreducible $3 \mathrm{NFs}$}

All the Feynman diagrams in the second and fourth rows of Fig. 6 can contain coupling matrices with two interaction-irreducible 3NFs. Again, for each different topology we list the expressions for forward-in-time contributions first, followed by the backward-in-time ones.

In the Goldstone diagrams of the term in Fig. 6i we have,

$$
\mathbf{M}_{r \alpha}^{\left(\mathrm{IIi}^{\prime}\right)} \equiv \frac{\sqrt{2}}{12} t_{k_{5} k_{6} k_{7}}^{n_{1} n_{2} n_{4}}\left(\mathcal{Y}_{\mu}^{k_{5}} \mathcal{Y}_{\nu}^{k_{6}} \mathcal{Y}_{\lambda}^{k_{7}} \mathcal{X}_{\rho}^{n_{4}}\right)^{*} \mathcal{Y}_{\eta}^{k_{3}} W_{\mu \nu \lambda, \alpha \eta \rho}
$$

which is antisymmetric in the indexes $n_{1}$ and $n_{2}$, and

$$
\mathbf{N}_{\alpha s}^{\left(\mathrm{IIi}^{\prime}\right)} \equiv \frac{\sqrt{2}}{12} W_{\alpha \eta \rho, \lambda \mu \nu} \mathcal{X}_{\eta}^{n_{3}}\left(\mathcal{Y}_{\rho}^{k_{7}} \mathcal{X}_{\lambda}^{n_{4}} \mathcal{X}_{\mu}^{n_{5}} \mathcal{X}_{\nu}^{n_{6}}\right)^{*} t_{k_{1} k_{2} k_{7}}^{n_{4} n_{5} n_{6}},
$$

which is antisymmetric in the indexes $k_{1}$ and $k_{2}$.

In the Goldstone diagrams of the term in Fig. 6p we have,

$$
\begin{aligned}
\mathbf{M}_{q \alpha}^{(\mathrm{IIp})} \equiv & \frac{\sqrt{3}}{12} \mathcal{A}_{45} \mathcal{P}_{123}\left(t_{k_{7} k_{8} k_{5}}^{n_{1} n_{2} n_{6}} \mathcal{X}_{\lambda}^{n_{3}} \mathcal{Y}_{\eta}^{k_{4}}\left(\mathcal{Y}_{\mu}^{k_{7}} \mathcal{Y}_{\nu}^{k_{8}} \mathcal{X}_{\rho}^{n_{6}}\right)^{*}\right. \\
& \left.W_{\mu \nu \lambda, \alpha \eta \rho}\right)
\end{aligned}
$$

and

$$
\begin{aligned}
\mathbf{N}_{\alpha u}^{(\mathrm{IIp})} \equiv & -\frac{\sqrt{3}}{12} W_{\alpha \eta \rho, \mu \nu \lambda} \mathcal{A}_{45} \mathcal{P}_{123}\left(\mathcal{X}_{\eta}^{n_{4}}\left(\mathcal{Y}_{\rho}^{k_{8}} \mathcal{X}_{\mu}^{n_{6}} \mathcal{X}_{\nu}^{n_{7}}\right)^{*}\right. \\
& \left.\mathcal{Y}_{\lambda}^{k_{3}} t_{k_{1} k_{2} k_{8}}^{n_{6} n_{7} n_{5}}\right) .
\end{aligned}
$$

In the Goldstone diagrams of the term in Fig. 6q we can also single out the following coupling matrices,

$$
\begin{aligned}
\mathbf{M}_{q \alpha}^{(\mathrm{IIq})} \equiv & -\frac{\sqrt{3}}{12} \mathcal{P}_{123}\left(t_{k_{4} k_{5} k_{8}}^{n_{1} n_{6} n_{7}}\left(\mathcal{Y}_{\mu}^{k_{8}} \mathcal{X}_{\eta}^{n_{6}} \mathcal{X}_{\rho}^{n_{7}}\right)^{*} \mathcal{X}_{\nu}^{n_{2}} \mathcal{X}_{\lambda}^{n_{3}}\right. \\
& \left.W_{\mu \nu \lambda, \alpha \eta \rho}\right)
\end{aligned}
$$

and

$$
\begin{aligned}
\mathbf{N}_{\alpha u}^{(\mathrm{IIq})} \equiv & \frac{\sqrt{3}}{12} W_{\alpha \eta \rho, \mu \nu \lambda} \mathcal{P}_{123}\left(\left(\mathcal{Y}_{\eta}^{k_{7}} \mathcal{Y}_{\rho}^{k_{8}} \mathcal{X}_{\mu}^{n_{6}}\right)^{*} \mathcal{Y}_{\nu}^{k_{2}} \mathcal{Y}_{\lambda}^{k_{3}}\right. \\
& \left.t_{k_{1} k_{7} k_{8}}^{n_{4} n_{5} n_{6}}\right) .
\end{aligned}
$$




\section{Formulas for $\mathbf{2 N F}$ interaction matrices with $3 p 2 h$ and $3 h 2 p$ configurations}

The interaction matrices that we have introduced in Sec. IIIB do not exhaust the list of all possible terms required for the $\mathrm{ADC}(3)$. A more complicated pattern in terms of ISCs is present in interaction matrices connecting $2 p 1 h$ and $3 p 2 h$ ISCs, as for the forward-in-time terms in the diagrams of Figs. 6d. 6g.

In the Feynman diagram of Fig. 6d one can find,

$$
\begin{aligned}
\mathbf{C}_{r q^{\prime}}^{p-p p h} \equiv & \frac{\sqrt{6}}{12} \mathcal{A}_{12} \mathcal{A}_{910} \mathcal{P}_{678}\left(\mathcal{X}_{\mu}^{n_{2}} \widetilde{V}_{\mu \nu, \lambda \rho}\left(\mathcal{X}_{\lambda}^{n_{7}} \mathcal{X}_{\rho}^{n_{8}} \mathcal{Y}_{\nu}^{k_{10}}\right)^{*}\right. \\
& \left.\delta_{n_{1} n_{6}} \delta_{k_{3} k_{9}}\right),
\end{aligned}
$$

while its complex conjugate term is contained in the diagram of Fig. 6f]

An interaction matrix connecting the same ISCs as the one in Eq. A21) is

$$
\begin{aligned}
\mathbf{C}_{r q^{\prime}}^{h-h h p} \equiv & \frac{\sqrt{6}}{12} \mathcal{A}_{678}\left(\mathcal{Y}_{\lambda}^{k_{3}} \widetilde{V}_{\mu \nu, \lambda \rho}\left(\mathcal{Y}_{\mu}^{k_{9}} \mathcal{Y}_{\nu}^{k_{10}} \mathcal{X}_{\rho}^{n_{8}}\right)^{*}\right. \\
& \left.\delta_{n_{1} n_{6}} \delta_{n_{2} n_{7}}\right),
\end{aligned}
$$

which is contained in the diagram in Fig. 6e, while the diagram in Fig. $6 \mathrm{~g}$ contains the complex conjugate of Eq. A22.

In the self-energy diagrams represented in Figs. 61 $6 \mathrm{n}$. when two $3 p 2 h$ ISCs interact through a $2 \mathrm{NF}$, we find interaction matrices of the following form:

$$
\begin{aligned}
\mathbf{C}_{q q^{\prime}}^{p p} \equiv & \frac{1}{12} \mathcal{A}_{45} \mathcal{P}_{123} \mathcal{P}_{678}\left(\mathcal{X}_{\mu}^{n_{1}} \mathcal{X}_{\nu}^{n_{2}} \widetilde{V}_{\mu \nu, \lambda \rho}\left(\mathcal{X}_{\lambda}^{n_{6}} \mathcal{X}_{\rho}^{n_{7}}\right)^{*}\right. \\
& \left.\delta_{n_{3} n_{8}} \delta_{k_{4} k_{9}} \delta_{k_{5} k_{10}}\right)
\end{aligned}
$$

which is composed by 18 terms when all the permutations indicated are taken, and the ones with a particle-hole $2 \mathrm{NF}$ connecting two $3 p 2 h$ propagators, i.e.

$$
\begin{aligned}
\mathbf{C}_{q q^{\prime}}^{p h} \equiv & \frac{1}{12} \mathcal{A}_{45} \mathcal{A}_{910} \mathcal{A}_{123} \mathcal{P}_{678}\left(\mathcal{X}_{\mu}^{n_{1}} \mathcal{Y}_{\rho}^{k_{4}} \widetilde{V}_{\mu \nu, \rho \lambda}\left(\mathcal{X}_{\lambda}^{n_{6}} \mathcal{Y}_{\nu}^{k_{9}}\right)^{*}\right. \\
& \left.\delta_{n_{2} n_{7}} \delta_{n_{3} n_{8}} \delta_{k_{5} k_{10}}\right),
\end{aligned}
$$

that contains 72 terms when the explicit antisymmetrization with respect to quasiparticle indexes is performed. The interaction matrices in Eqs. A23) and (A24) are found in the diagrams in Figs. 61 and $6 \mathrm{~m}$, respectively.

A forward-in-time interaction matrix connecting two $3 p 2 h$ ISCs through a hole-hole $2 \mathrm{NF}$ is found in the selfenergy diagram in Fig. 6n. This has the following expression,

$$
\begin{aligned}
\mathbf{C}_{q q^{\prime}}^{h h} \equiv & \frac{1}{12} \mathcal{A}_{123}\left(\mathcal{Y}_{\lambda}^{k_{4}} \mathcal{Y}_{\rho}^{k_{5}} \widetilde{V}_{\mu \nu, \lambda \rho}\left(\mathcal{Y}_{\mu}^{k_{9}} \mathcal{Y}_{\nu}^{k_{10}}\right)^{*}\right. \\
& \left.\delta_{n_{1} n_{6}} \delta_{n_{2} n_{7}} \delta_{n_{3} n_{8}}\right)
\end{aligned}
$$

We present now the corresponding interaction matrices appearing in backward-in-time self-energy Goldstone diagrams. We remind that these interaction matrices are the ones connecting propagators of multihole-multiparticle type in self-energy diagrams.

We consider first terms contained in Figs. 6d 6g, namely those connecting the $2 h 1 p$ propagator to the $3 h 2 p$ propagator. We find,

$$
\begin{aligned}
\mathbf{D}_{s u^{\prime}}^{h-h h p} \equiv & \frac{\sqrt{6}}{12} \mathcal{A}_{12} \mathcal{A}_{910} \mathcal{P}_{678}\left(\left(\mathcal{Y}_{\mu}^{k_{2}}\right)^{*} \widetilde{V}_{\mu \nu, \lambda \rho} \mathcal{Y}_{\lambda}^{k_{7}} \mathcal{Y}_{\rho}^{k_{8}} \mathcal{X}_{\nu}^{n_{10}}\right. \\
& \left.\delta_{k_{1} k_{6}} \delta_{n_{3} n_{9}}\right),
\end{aligned}
$$

which must be combined with another interaction matrix,

$\mathbf{D}_{s u^{\prime}}^{p-p p h} \equiv-\frac{\sqrt{6}}{12} \mathcal{A}_{678}\left(\left(\mathcal{X}_{\lambda}^{n_{3}}\right)^{*} \widetilde{V}_{\mu \nu, \lambda \rho} \mathcal{X}_{\mu}^{n_{9}} \mathcal{X}_{\nu}^{n_{10}} \mathcal{Y}_{\rho}^{k_{8}} \delta_{k_{1} k_{6}} \delta_{k_{2} k_{7}}\right)$.

Interaction matrices in Eqs. (A26) and A27) are found in the self-energy diagrams of Figs. 6d and 6e respectively, while their complex conjugates are contained in the diagrams of Figs. 6f and $6 \mathrm{~g}$.

When two $3 h 2 p$ propagators in a self-energy diagram are connected via ISCs linked to 2NFs, as in diagrams of Figs. 61. 6n, we have an interaction matrix of the following form,

$$
\begin{aligned}
\mathbf{D}_{u u^{\prime}}^{h h} \equiv & -\frac{1}{12} \mathcal{A}_{45} \mathcal{P}_{123} \mathcal{P}_{678}\left(\left(\mathcal{Y}_{\mu}^{k_{1}} \mathcal{Y}_{\nu}^{k_{2}}\right)^{*} \widetilde{V}_{\mu \nu, \lambda \rho} \mathcal{Y}_{\lambda}^{k_{6}} \mathcal{Y}_{\rho}^{k_{7}}\right. \\
& \left.\delta_{k_{3} k_{8}} \delta_{n_{4} n_{9}} \delta_{n_{5} n_{10}}\right),
\end{aligned}
$$

contained in the diagram of Fig. 61, and another one found when a particle-hole $2 \mathrm{NF}$ connects two $3 h 2 p$ ISCs, i.e.

$$
\begin{aligned}
\mathbf{D}_{u u^{\prime}}^{h p} \equiv & -\frac{1}{12} \mathcal{A}_{45} \mathcal{A}_{910} \mathcal{A}_{123} \mathcal{P}_{678}\left(\left(\mathcal{Y}_{\mu}^{k_{1}} \mathcal{X}_{\rho}^{n_{4}}\right)^{*} \widetilde{V}_{\mu \nu, \rho \lambda}\right. \\
& \left.\mathcal{Y}_{\lambda}^{k_{6}} \mathcal{X}_{\nu}^{n_{9}} \delta_{k_{2} k_{7}} \delta_{k_{3} k_{8}} \delta_{n_{5} n_{10}}\right),
\end{aligned}
$$

which appears in the Feynman diagram of Fig. 6m.

Finally, also a backward-in-time interaction matrix connecting two $3 h 2 p$ ISCs through a particle-particle $2 \mathrm{NF}$ is found in the self-energy diagram of Fig. 6n, that is

$$
\begin{aligned}
\mathbf{D}_{u u^{\prime}}^{p p} \equiv & -\frac{1}{12} \mathcal{A}_{123}\left(\left(\mathcal{X}_{\lambda}^{n_{4}} \mathcal{X}_{\rho}^{n_{5}}\right)^{*} \widetilde{V}_{\mu \nu, \lambda \rho} \mathcal{X}_{\mu}^{n_{9}} \mathcal{X}_{\nu}^{n_{10}}\right. \\
& \left.\delta_{k_{1} k_{6}} \delta_{k_{2} k_{7}} \delta_{k_{3} k_{8}}\right)
\end{aligned}
$$

\section{Formulas for $\mathbf{3 N F}$ interaction matrices with $3 p 2 h$ and $3 h 2 p$ configurations}

The last set of interaction matrices is required to complete the $\mathrm{ADC}(3)$, which is given by those terms containing the interaction-irreducible $3 \mathrm{NF}$. 
First we consider interaction matrices in which the $3 \mathrm{NF}$ connects $2 p 1 h$ ISCs to $3 p 2 h$ ISCs, as in the diagrams in Figs. 6h 6h. We find in the diagram of Fig. 6h.

$$
\begin{aligned}
\mathbf{C}_{r q^{\prime}}^{3 N(I)} \equiv & \frac{\sqrt{6}}{12} \mathcal{A}_{12} \mathcal{P}_{678}\left(\delta_{n_{2} n_{7}} \mathcal{X}_{\lambda}^{n_{1}} \mathcal{Y}_{\eta}^{k_{3}} W_{\lambda \mu \nu, \rho \eta \epsilon}\right. \\
& \left.\left(\mathcal{X}_{\rho}^{n_{6}} \mathcal{X}_{\epsilon}^{n_{8}} \mathcal{Y}_{\mu}^{k_{9}} \mathcal{Y}_{\nu}^{k_{10}}\right)^{*}\right),
\end{aligned}
$$

and a second $3 \mathrm{NF}$ interaction matrix connecting the $2 p 1 \mathrm{~h}$ propagator to the $3 p 2 h$ propagator, requiring an explicit antisymmetrization only with respect to two particle indexes. Its expression from a Goldstone diagram of Fig. 6i reads,

$$
\begin{aligned}
\mathbf{C}_{r q^{\prime}}^{3 N(I I)} \equiv & \frac{\sqrt{6}}{12} \mathcal{A}_{910}\left(\mathcal{X}_{\lambda}^{n_{1}} \mathcal{X}_{\nu}^{n_{2}} W_{\lambda \nu \mu, \rho \epsilon \eta}\right. \\
& \left.\left(\mathcal{X}_{\rho}^{n_{6}} \mathcal{X}_{\epsilon}^{n_{7}} \mathcal{X}_{\eta}^{n_{8}} \mathcal{Y}_{\mu}^{k_{10}}\right)^{*} \delta_{k_{3} k_{9}}\right) .
\end{aligned}
$$

Complex conjugate interaction matrices corresponding to Eqs. A31 and A32 can be found in the Goldstone diagrams of Figs. 6j and $6 \mathrm{k}$

Two $3 p 2 h$ propagators can be connected in a selfenergy diagram via ISCs containing a $3 \mathrm{NF}$. They are contained in the three self-energy diagrams represented in Figs. 60 6q We have then an interaction matrix of the following form in Fig. 60,

$$
\begin{aligned}
\mathbf{C}_{q q^{\prime}}^{3 N(I I I)} \equiv & \frac{1}{12} \mathcal{A}_{45}\left(\mathcal{X}_{\lambda}^{n_{1}} \mathcal{X}_{\nu}^{n_{2}} \mathcal{X}_{\mu}^{n_{3}} W_{\lambda \nu \mu, \rho \epsilon \eta}\left(\mathcal{X}_{\rho}^{n_{6}} \mathcal{X}_{\epsilon}^{n_{7}} \mathcal{X}_{\eta}^{n_{8}}\right)^{*}\right. \\
& \left.\delta_{k_{4} k_{9}} \delta_{k_{5} k_{10}}\right) .
\end{aligned}
$$

Other $3 \mathrm{NF}$ interaction matrices connecting two $3 p 2 h$ ISCs are found in Fig. 6p.

$$
\begin{array}{r}
\mathbf{C}_{q q^{\prime}}^{3 N(I V)} \equiv-\frac{1}{12} \mathcal{A}_{45} \mathcal{A}_{910} \mathcal{P}_{123} \mathcal{P}_{678}\left(\mathcal{X}_{\lambda}^{n_{1}} \mathcal{X}_{\nu}^{n_{2}} \mathcal{Y}_{\eta}^{k_{5}}\right. \\
\left.W_{\lambda \nu \mu, \rho \epsilon \eta}\left(\mathcal{X}_{\rho}^{n_{6}} \mathcal{X}_{\epsilon}^{n_{7}} \mathcal{Y}_{\mu}^{k_{10}}\right)^{*} \delta_{k_{4} k_{9}} \delta_{n_{3} n_{8}}\right)
\end{array}
$$

and in Fig. 6q

$$
\begin{aligned}
\mathbf{C}_{q q^{\prime}}^{3 N(V)} \equiv & \frac{1}{12} \mathcal{A}_{123} \mathcal{P}_{678}\left(\mathcal{X}_{\mu}^{n_{1}} \mathcal{Y}_{\epsilon}^{k_{4}} \mathcal{Y}_{\eta}^{k_{5}} W_{\mu \nu \lambda, \rho \epsilon \eta}\right. \\
& \left.\left(\mathcal{X}_{\rho}^{n_{6}} \mathcal{Y}_{\nu}^{k_{9}} \mathcal{Y}_{\lambda}^{k_{10}}\right)^{*} \delta_{n_{2} n_{7}} \delta_{n_{3} n_{8}}\right) .
\end{aligned}
$$

We can present now 3NF interaction matrices appearing in backward-in-time self-energy Goldstone diagrams. These interaction matrices connect hole-particle ISCs in the diagrams, i.e. $2 h 1 p$ and/or $3 h 2 p$ propagators. As the corresponding interaction matrices for the forwardin-time part shown above, they are found in the diagrams of Figs. 6h 6h and Figs. 6o 6a

First we have the terms,

$$
\begin{aligned}
\mathbf{D}_{s u^{\prime}}^{3 N(I)} \equiv & -\frac{\sqrt{6}}{12} \mathcal{P}_{12} \mathcal{P}_{678}\left(\delta_{k_{2} k_{7}}\left(\mathcal{Y}_{\lambda}^{k_{1}} \mathcal{X}_{\eta}^{n_{3}}\right)^{*} W_{\lambda \nu \mu, \rho \eta \epsilon}\right. \\
& \left.\mathcal{Y}_{\rho}^{k_{6}} \mathcal{Y}_{\epsilon}^{k_{8}} \mathcal{X}_{\nu}^{n_{9}} \mathcal{X}_{\mu}^{n_{10}}\right),
\end{aligned}
$$

and

$$
\begin{aligned}
\mathbf{D}_{s u^{\prime}}^{3 N(I I)} \equiv & -\frac{\sqrt{6}}{12} \mathcal{A}_{910}\left(\left(\mathcal{Y}_{\lambda}^{k_{1}} \mathcal{Y}_{\nu}^{k_{2}}\right)^{*} W_{\lambda \nu \mu, \rho \epsilon \eta}\right. \\
& \left.\mathcal{Y}_{\rho}^{k_{6}} \mathcal{Y}_{\epsilon}^{k_{7}} \mathcal{Y}_{\eta}^{k_{8}} \mathcal{X}_{\mu}^{n_{10}} \delta_{n_{3} n_{9}}\right)
\end{aligned}
$$

which are found in Figs. 6h and 6i, respectively.

Finally, 3NF interaction matrices can connect two $3 h 2 p$ ISCs within a self-energy diagram, as in diagrams of Figs. 6o-6q Specifically, in Figs. 6o and 6p we can single out the interaction matrices,

$$
\begin{aligned}
\mathbf{D}_{u u^{\prime}}^{3 N(I I I)} \equiv & \frac{1}{12} \mathcal{A}_{45}\left(\left(\mathcal{Y}_{\lambda}^{k_{1}} \mathcal{Y}_{\nu}^{k_{2}} \mathcal{Y}_{\mu}^{k_{3}}\right)^{*} W_{\lambda \nu \mu, \rho \epsilon \eta} \mathcal{Y}_{\rho}^{k_{6}} \mathcal{Y}_{\epsilon}^{k_{7}} \mathcal{Y}_{\eta}^{k_{8}}\right. \\
& \left.\delta_{n_{4} n_{9}} \delta_{n_{5} n_{10}}\right),
\end{aligned}
$$

and

$$
\begin{aligned}
\mathbf{D}_{u u^{\prime}}^{3 N(I V)} \equiv & -\frac{1}{12} \mathcal{A}_{45} \mathcal{A}_{910} \mathcal{P}_{123} \mathcal{P}_{678}\left(\left(\mathcal{Y}_{\lambda}^{k_{1}} \mathcal{Y}_{\nu}^{k_{2}} \mathcal{X}_{\eta}^{n_{5}}\right)^{*}\right. \\
& \left.W_{\lambda \nu \mu, \rho \epsilon \eta} \mathcal{Y}_{\rho}^{k_{6}} \mathcal{Y}_{\epsilon}^{k_{7}} \mathcal{X}_{\mu}^{n_{10}} \delta_{n_{4} n_{9}} \delta_{k_{3} k_{8}}\right),
\end{aligned}
$$

respectively. Matrices in Eqs. A38 and A39 must be complemented with another backward-in-time interaction matrix that also connects two $3 h 2 p$ ISCs. This is given by the expression,

$$
\begin{aligned}
\mathbf{D}_{u u^{\prime}}^{3 N(V)} \equiv & \frac{1}{12} \mathcal{A}_{123} \mathcal{P}_{678}\left(\left(\mathcal{Y}_{\mu}^{k_{1}} \mathcal{X}_{\epsilon}^{n_{4}} \mathcal{X}_{\eta}^{n_{5}}\right)^{*} W_{\mu \nu \lambda, \rho \epsilon \eta}\right. \\
& \left.\mathcal{Y}_{\rho}^{k_{6}} \mathcal{X}_{\nu}^{n_{9}} \mathcal{X}_{\lambda}^{n_{10}} \delta_{k_{2} k_{7}} \delta_{k_{3} k_{8}}\right)
\end{aligned}
$$

as it is singled out from the Goldstone diagram corresponding to Fig. 6q

\section{Appendix B: Irreducible self-energy in angular momentum coupling formalism}

Most implementations of $a b$ initio approaches in nuclear physics are based on the assumption of a spherical ground state, so that one can exploit a spherical single-particle basis and the angular momentum formalism to decouple different channels. The diagonalization of Eq. (28) can then be performed separately for each partial wave, and the required computational resources are significantly reduced. In this Appendix we follow this path and derive the corresponding working equations for $\mathrm{ADC}(3)$ and $3 \mathrm{NFs}$ including up to $2 p 1 h$ and $2 h 1 p$ ISCs. The ADC(3) matrix elements for 2NFs have been discussed in the past 39 and used in applications for several years [11, 40, 41]. However, they were never published in detail for the case of fully dressed propagators. Moreover, we complement them with the new terms arising from 3NFs. We present them here in the hope that they might turn out to be useful to practitioners.

In general, a spherical single-particle state with isospin function $\chi_{q}$, is given by coupling the spherical harmonic 
$Y_{l}(\hat{r})$ to $\chi_{\frac{1}{2}}$, the function of the intrinsic spin of the nucleon,

$$
\phi_{\beta}(\vec{r}, \sigma, \tau)=f_{n_{\beta} l_{\beta}}(r)\left[Y_{l_{\beta}}(\hat{r}) \otimes \chi_{\frac{1}{2}}(\sigma)\right]_{j_{\beta}}^{m_{\beta}} \chi_{q_{\beta}}(\tau),
$$

with $\sigma$ and $\tau$ being spin and isospin coordinates, respectively.

The collective index $\beta$ denotes the set of quantum numbers $\left(n_{\beta}, \pi_{\beta}, j_{\beta}, m_{\beta}, q_{\beta}\right)$, where $n_{\beta}$ is the principal quantum number, $\pi_{\beta}$ is the parity corresponding to the orbital angular momentum $l_{\beta}, j_{\beta}$ and $m_{\beta}$ are the total angular momentum and its projection along the $z$ axis, and $q_{\beta}$ represents the isospin projection. In this basis, the creation operator $a_{\beta}^{\dagger}$ of the single-particle basis is the $m_{\beta}^{\text {th }}$ component of an irreducible tensor of rank $j_{\beta}$ :

$$
a_{\beta}^{\dagger} \equiv a_{n_{\beta}, \pi_{\beta}, j_{\beta}, m_{\beta}, q_{\beta}}^{\dagger} \equiv a_{b, m_{\beta}}^{\dagger},
$$

where we made use of the notation $\beta=\left(b, m_{\beta}\right)$, i.e.

$$
b \equiv\left(n_{\beta}, \pi_{\beta}, j_{\beta}, q_{\beta}\right)
$$

The destruction operators are dealt with in the same fashion but we add a phase factor and invert the quantum number $m$, which is needed to obtain an irreducible tensor $\bar{a}_{\beta} \equiv(-1)^{j_{\beta}+m_{\beta}} a_{b,-m_{\beta}}$. For particle and hole Dyson orbits, corresponding to the eigenstates $\left|\Psi_{n}^{A+1}\right\rangle$ and $\left|\Psi_{k}^{A-1}\right\rangle$ of the $(\mathrm{A}+1)$ - and (A-1)body systems, we use a compact notation analogous to Eq. B2 and write $n=\left(\tilde{n}, m_{n}\right)$ and $k=\left(\tilde{k}, m_{k}\right)$, with $\tilde{n} \equiv\left(n_{n}, \pi_{n}, j_{n}, q_{n}\right)$ and $\tilde{k} \equiv\left(n_{k}, \pi_{k}, j_{k}, q_{k}\right)$. Using these

definitions, the shorthand notations of Eqs. 20 and 21 can be coupled to total angular momentum and the overall quantum number $M$ is separated. This leads us to define $\tilde{r} \equiv\left[\left(\tilde{n}_{1}, \tilde{n}_{2}, J_{12}, \tilde{k}_{3}\right), J_{r}\right], \tilde{s} \equiv\left[\left(\tilde{k}_{1}, \tilde{k}_{2}, J_{12}, \tilde{n}_{3}\right), J_{s}\right]$ and so on, where we follow the coupling conventions of Eqs. B12 and (B13) below.

We now revisit the angular momentum coupling of the self-energy, when the ground state $\left|\Psi_{0}^{A}\right\rangle$ in Eq. (1) has total angular momentum and parity $J^{\pi}=0^{+}$. For these systems, the formalism is considerably simplified because the total angular momentum $j_{n}\left(j_{k}\right)$, its projection along the $z$ axis $m_{n}\left(m_{k}\right)$ and the parity $\pi_{n}$ $\left(\pi_{k}\right)$ of excited states $\Psi_{n}^{A+1}\left(\Psi_{k}^{A-1}\right)$ are the same as the corresponding quantum numbers of the particle creation (annihilation) tensor operators entering the definition of the Green's function, Eq. (1). The total isospin is also uniquely determined by isospin projections of the reference state and tensor operators. With our assumption that $\left|\Psi_{0}^{A}\right\rangle$ has $J^{\pi}=0^{+}$, the irreducible self-energy in the Dyson equation (7) becomes diagonal in the quantum numbers $(\pi, j, m, q)$, and it is independent on $m$ :

$$
\Sigma_{\alpha \beta}^{\star}(\omega)=\delta_{a b}^{(\pi j q)} \delta_{m_{\alpha} m_{\beta}} \Sigma_{a b}^{\star}(\omega)
$$

where we have introduced a compact notation for multiple Kronecker deltas:

$$
\delta_{a b}^{(\pi j q)} \equiv \delta_{\pi_{\alpha} \pi_{\beta}} \delta_{j_{\alpha} j_{\beta}} \delta_{q_{\alpha} q_{\beta}}
$$

By applying the Wigner-Eckart theorem to the transition amplitudes from Eq. (6), one finds:

$$
\mathcal{X}_{\alpha}^{\tilde{n}, m_{n}}=(-1)^{2 j_{n}} \frac{\delta_{a \tilde{n}}^{(\pi j q)} \delta_{m_{\alpha} m_{n}}}{\sqrt{2 j_{\alpha}+1}}\left\langle\Psi_{\tilde{n}}^{A+1}\left\|a_{a}^{\dagger}\right\| \Psi_{0}^{A}\right\rangle \equiv \mathcal{X}_{a}^{\tilde{n}} \delta_{a \tilde{n}}^{(\pi j q)} \delta_{m_{\alpha} m_{n}}
$$

and

$$
\mathcal{Y}_{\alpha}^{\tilde{k}, m_{k}}=(-1)^{2 j_{k}}(-1)^{j_{\alpha}-m_{\alpha}} \frac{\delta_{a \tilde{k}}^{(\pi j q)} \delta_{-m_{\alpha} m_{k}}}{\sqrt{2 j_{\alpha}+1}}\left\langle\Psi_{\tilde{k}}^{A-1}|| \bar{a}_{a} \| \Psi_{0}^{A}\right\rangle \equiv \mathcal{Y}_{a}^{\tilde{k}}(-1)^{j_{\alpha}-m_{\alpha}} \delta_{a \tilde{k}}^{(\pi j q)} \delta_{-m_{\alpha} m_{k}},
$$

which define the $m$-independent spectroscopic amplitudes $\mathcal{X}_{a}^{\tilde{n}}$ and $\mathcal{Y}_{a}^{\tilde{k}}$.

Because of rotational invariance of the Hamiltonian, the 2NFs and 3NFs are coupled as $m$-independent matrix elements according to

$$
\bar{V}_{a b, g d}^{J}=\frac{1}{\sqrt{1+\delta_{a b}} \sqrt{1+\delta_{g d}}} \sum_{\substack{m_{\alpha} m_{\beta} \\ m_{\gamma} m_{\delta}}}\left(j_{\alpha} j_{\beta} m_{\alpha} m_{\beta} \mid J M\right)\left(j_{\gamma} j_{\delta} m_{\gamma} m_{\delta} \mid J M\right) \tilde{V}_{\alpha \beta, \gamma \delta}
$$

and

$$
W_{a b l, g d v}^{J_{1} J_{2} J}=\sum_{\substack{m_{\alpha} m_{\beta} \\ m_{\lambda}}} \sum_{\substack{m_{\gamma} m_{\delta} \\ m_{\nu}}}\left(j_{\alpha} j_{\beta} m_{\alpha} m_{\beta} \mid J_{1} M_{1}\right)\left(j_{\gamma} j_{\delta} m_{\gamma} m_{\delta} \mid J_{2} M_{2}\right)\left(J_{1} j_{\lambda} M_{1} m_{\lambda} \mid J M\right)\left(J_{2} j_{\nu} M_{2} m_{\nu} \mid J M\right) W_{\alpha \beta \lambda, \gamma \delta \nu}
$$

Note that we chose to properly normalize the matrix elements of $\bar{V}$ in Eq. (B8) but not those of $W$. When considering the Goldstone diagrams that result from Fig. $2 \mathrm{c}$, it is also convenient to recouple the angular momenta of the $3 \mathrm{NF}$ in 
the particle-particle-hole channel:

$$
\begin{aligned}
W_{a b l^{-1}, g d v^{-1}}^{(p p h) J_{1} J_{2} J}=- & \sum_{\substack{m_{\alpha} m_{\beta} \\
m_{\lambda}}} \sum_{\substack{m_{\gamma} m_{\delta} \\
m_{\nu}}}\left(j_{\alpha} j_{\beta} m_{\alpha} m_{\beta} \mid J_{1} M_{1}\right)\left(j_{\gamma} j_{\delta} m_{\gamma} m_{\delta} \mid J_{2} M_{2}\right) \\
& \quad \times\left(J_{1} j_{\lambda} M_{1}-m_{\lambda} \mid J M\right)\left(J_{2} j_{\nu} M_{2}-m_{\nu} \mid J M\right)(-)^{j_{\nu}-m_{\nu}}(-)^{j_{\lambda}-m_{\lambda}} W_{\alpha \beta \nu, \gamma \delta \lambda} .
\end{aligned}
$$

The matrix elements $W$ can be transformed into the $W^{(p p h)}$ form by using the Pandya relation [42]:

$$
W_{a b l^{-1}, g d v^{-1}}^{(p p h) J_{1} J_{2} J}=-(-)^{2 j_{\nu}+2 j_{\lambda}+2 J} \sum_{J^{\prime}}\left(2 J^{\prime}+1\right)\left\{\begin{array}{c}
J_{1} j_{\lambda} J \\
J_{2} j_{\nu} J^{\prime}
\end{array}\right\} W_{a b v, g d l}^{J_{1} J_{2} J^{\prime}} .
$$

\section{General J-coupling conventions for ISCs}

The ADC coupling and interaction matrices derived in earlier sections already separate naturally in terms of the parity and isospin (i.e., charge differences with respect to the ground state $\left|\Psi_{0}^{A}\right\rangle$ ). To decouple the Dyson eigenvalue problem according to Eq. (B4), we then need to recouple the ISCs to total angular momentum. In this Appendix we are going to consider only the ADC contributions arising from Feynman diagrams of Fig. 2, Thus, we need to specify an angular momentum coupling convention for $2 p 1 h$ and $2 h 1 p$ ISCs. As usual, the particular choice of coupling may introduce particular phases and factors in the equations but the final result of the Dyson diagonalization, Eq. (28), does not depend on these.

For $2 p 1 h$ coupling matrices we define the coupled elements of $\mathbf{M}_{\tilde{r} a}$ as

$$
\sum_{\substack{m_{n_{1}} m_{n_{2}} \\ m_{k_{3}}}}\left(j_{n_{1}} j_{n_{2}} m_{n_{1}} m_{n_{2}} \mid J_{12} M_{12}\right)\left(J_{12} j_{k_{3}} M_{12} m_{k_{3}} \mid J_{r} M_{r}\right) \mathbf{M}_{r \alpha} \equiv \delta_{a r}^{(\pi j q)} \delta_{m_{\alpha} M_{r}} \sqrt{\frac{1+\delta_{\tilde{n}_{1} \tilde{n}_{2}}}{2}} \mathbf{M}_{\tilde{r} a}
$$

with the parity $\pi_{r}=(-1)^{l_{1}+l_{2}+l_{3}}$, the charge $q_{r}=q_{1}+q_{2}-q_{3}$ and the total angular momentum $J_{r}$ of the $2 p 1 h$ ISC $\tilde{r}=\left[\left(\tilde{n}_{1}, \tilde{n}_{2}, J_{12}, \tilde{k}_{3}\right), J_{r}\right]$. For the backward-in-time $2 h 1 p$ matrix $\mathbf{N}_{\alpha \tilde{s}}$, we write:

$$
\sum_{\substack{m_{k_{1}} m_{k_{2}} \\ m_{n_{3}}}}\left(j_{k_{1}} j_{k_{2}} m_{k_{1}} m_{k_{2}} \mid J_{12} M_{12}\right)\left(J_{12} j_{n_{3}} M_{12} m_{n_{3}} \mid J_{s} M_{s}\right) \mathbf{N}_{\alpha s} \equiv \delta_{a s}^{(\pi j q)} \delta_{m_{\alpha}-M_{s}} \sqrt{\frac{1+\delta_{\tilde{k}_{1} \tilde{k}_{2}}}{2} \mathbf{N}_{a \tilde{s}}}
$$

with the charge $q_{s}=q_{1}+q_{2}-q_{3}$ and the total angular momentum $J_{s}$ of the $2 h 1 p$ ISC $\tilde{s}=\left[\left(\tilde{k}_{1}, \tilde{k}_{2}, J_{12}, \tilde{n}_{3}\right), J_{s}\right]$. The interaction matrices $\mathbf{C}_{j j^{\prime}}$ and $\mathbf{D}_{k k^{\prime}}$ are coupled in the same way. For $2 p 1 h$ matrix elements $\mathbf{C}_{r r^{\prime}}$, we have

$$
\begin{gathered}
\sum_{\substack{m_{n_{1}} m_{n_{2}} \\
m_{k_{3}}}} \sum_{\substack{m_{n_{4}} m_{n_{5}} \\
m_{k_{6}}}}\left(j_{n_{1}} j_{n_{2}} m_{n_{1}} m_{n_{2}} \mid J_{12} M_{12}\right)\left(J_{12} j_{k_{3}} M_{12} m_{k_{3}} \mid J_{r} M_{r}\right)\left(j_{n_{4}} j_{n_{5}} m_{n_{4}} m_{n_{5}} \mid J_{45} M_{45}\right)\left(J_{45} j_{k_{6}} M_{45} m_{k_{6}} \mid J_{r^{\prime}} M_{r^{\prime}}\right) \mathbf{C}_{r r^{\prime}} \\
=\delta_{r r^{\prime}}^{(\pi j q)} \delta_{M_{r} M_{r^{\prime}}} \sqrt{\frac{1+\delta_{\tilde{n}_{1} \tilde{n}_{2}}}{2}} \mathbf{C}_{\tilde{r} \tilde{r}^{\prime}} \sqrt{\frac{1+\delta_{\tilde{n}_{4} \tilde{n}_{5}}}{2}}
\end{gathered}
$$

And for the $2 h 1 p$ matrix elements $\mathbf{D}_{s s^{\prime}}$ :

$$
\begin{gathered}
\sum_{\substack{m_{k_{1}} m_{k_{2}} \\
m_{n_{3}}}} \sum_{\substack{m_{k_{4}} m_{k_{5}} \\
m_{n_{6}}}}\left(j_{k_{1}} j_{k_{2}} m_{k_{1}} m_{k_{2}} \mid J_{12} M_{12}\right)\left(J_{12} j_{n_{3}} M_{12} m_{n_{3}} \mid J_{s} M_{s}\right)\left(j_{k_{4}} j_{k_{5}} m_{k_{4}} m_{k_{5}} \mid J_{45} M_{45}\right)\left(J_{45} j_{n_{6}} M_{45} m_{n_{6}} \mid J_{s^{\prime}} M_{s^{\prime}}\right) \mathbf{D}_{s s^{\prime}} \\
=\delta_{s s^{\prime}}^{(\pi j q)} \delta_{M_{s} M_{s^{\prime}}} \sqrt{\frac{1+\delta_{\tilde{k}_{1} \tilde{k}_{2}}}{2}} \mathbf{D}_{\tilde{s} \tilde{s}^{\prime}} \sqrt{\frac{1+\delta_{\tilde{k}_{4} \tilde{k}_{5}}}{2}}
\end{gathered}
$$

Equations (B14) and B15 define the coupled $\mathbf{C}_{\tilde{r} \tilde{r}^{\prime}}$ and $\mathbf{D}_{\tilde{s} \tilde{s}^{\prime}}$ that are independent of $M_{r}$ and $M_{s}$, respectively. With the above definitions, the unperturbed energies for each ISC do not depend on the angular momentum coupling and their expressions, Eqs. 22 and (23), remain unchanged. For example:

$$
\mathbf{E}_{\tilde{r} \tilde{r}^{\prime}}=\operatorname{diag}\left\{\varepsilon_{\tilde{n}_{1}}^{+}+\varepsilon_{\tilde{n}_{2}}^{+}-\varepsilon_{\tilde{k}_{3}}^{-}\right\}
$$

Note that the coupling and interaction matrices introduced in Secs. III A and III B are explicitly antisymmetric for the exchanges in any of the particle $\left(n_{1}, n_{2}, n_{3}, \ldots\right)$ or any of the hole $\left(k_{1}, k_{2}, k_{3}, \ldots\right)$ indices. They are nevertheless defined 
assuming unrestricted sum over all indexes. In practical calculations, one can drop the symmetry factors of $1 / \sqrt{2}$ for $2 p 1 h(2 h 1 p)$ ISCs and restrict the sums over the fist two indexes, $n_{1}<n_{2}\left(k_{1}<k_{2}\right)$. In angular momentum coupling formalism one has similarly ordered sums- $\tilde{n}_{1} \leq \tilde{n}_{2}$ and $\tilde{k}_{1} \leq \tilde{k}_{2}$ in this case -and factors such as $\sqrt{\left(1+\delta_{\tilde{n}_{1}} \tilde{n}_{2}\right) / 2}$ are to be dropped. Thus, we separated these factors already in the definitions $(\mathrm{B} 12)-(\bar{B} 15)$.

With the above definitions, and assuming ordered sums, the dynamic self-energy of Eq. 19p can be decoupled as

$$
\begin{aligned}
& \widetilde{\Sigma}_{\alpha \beta}(\omega)=\sum_{r r^{\prime}} \mathbf{M}_{\alpha r}^{*} \frac{1}{\hbar \omega-\left(\mathbf{E}_{r} \delta_{r r^{\prime}}+\mathbf{C}_{r r^{\prime}}\right)+\mathrm{i} \eta} \mathbf{M}_{r^{\prime} \beta}+\sum_{s s^{\prime}} \mathbf{N}_{\alpha s} \frac{1}{\hbar \omega-\left(\mathbf{E}_{s} \delta_{s s^{\prime}}+\mathbf{D}_{s s^{\prime}}\right)-\mathrm{i} \eta} \mathbf{N}_{s^{\prime} \beta}^{*} \\
& =\sum_{\tilde{r} \tilde{r}^{\prime} M_{r} M_{r^{\prime}}} \delta_{a r}^{(\pi j q)} \delta_{m_{\alpha} M_{r}} \mathbf{M}_{a \tilde{r}}^{*} \frac{1}{\hbar \omega-\left(\mathbf{E}_{\tilde{r}} \delta_{\tilde{r} \tilde{r}^{\prime}}+\mathbf{C}_{\tilde{r} \tilde{r}^{\prime}}\right) \delta_{r r^{\prime}}^{(\pi j q)} \delta_{M_{r} M_{r^{\prime}}}+\mathrm{i} \eta} \delta_{b r^{\prime}}^{(\pi j q)} \delta_{m_{\beta} M_{r^{\prime}}} \mathbf{M}_{\tilde{r}^{\prime} b} \\
& +\sum_{\tilde{s} \tilde{s}^{\prime} M_{s} M_{s^{\prime}}} \delta_{a s}^{(\pi j q)} \delta_{m_{\alpha}-M_{s}} \mathbf{N}_{a \tilde{s}} \frac{1}{\hbar \omega-\left(\mathbf{E}_{\tilde{s}} \delta_{\tilde{s} \tilde{s}^{\prime}}+\mathbf{D}_{\tilde{s} \tilde{s}^{\prime}}\right) \delta_{s s^{\prime}}^{(\pi j q)} \delta_{M_{s} M_{s^{\prime}}-\mathrm{i} \eta}} \delta_{b s^{\prime}}^{(\pi j q)} \delta_{m_{\beta}-M_{s^{\prime}}} \mathbf{N}_{b \tilde{s}^{\prime}}^{*} \\
& =\delta_{a b}^{(\pi j q)} \delta_{m_{\alpha} m_{\beta}}\left(\sum_{\tilde{r} \tilde{r}^{\prime}} \delta_{a r}^{(\pi j q)} \mathbf{M}_{a \tilde{r}}^{*} \frac{1}{\hbar \omega-\left(\mathbf{E}_{\tilde{r}} \delta_{\tilde{r} \tilde{r}^{\prime}}+\mathbf{C}_{\tilde{r} \tilde{r}^{\prime}}\right)+\mathrm{i} \eta} \delta_{b r^{\prime}}^{(\pi j q)} \mathbf{M}_{\tilde{r}^{\prime} b}\right. \\
& \left.+\sum_{\tilde{s} \tilde{s}^{\prime}} \delta_{a s}^{(\pi j q)} \mathbf{N}_{a \tilde{s}} \frac{1}{\hbar \omega-\left(\mathbf{E}_{\tilde{s}} \delta_{\tilde{s} \tilde{s}^{\prime}}+\mathbf{D}_{\tilde{s} \tilde{s}^{\prime}}\right)-\mathrm{i} \eta} \delta_{b s^{\prime}}^{(\pi j q)} \mathbf{N}_{b \tilde{s}^{\prime}}^{*}\right) \\
& \equiv \delta_{a b}^{(\pi j q)} \delta_{m_{\alpha} m_{\beta}} \widetilde{\Sigma}_{a b}(\omega),
\end{aligned}
$$

which proves the energy dependent part of Eq. (B4) and defines the $m$-independent irreducible self-energy $\Sigma_{a b}^{\star}(\omega)$.

\section{Angular momentum coupling of the $\mathrm{ADC}(3)$ equations}

We are now in the position to give expressions for the coupling and interaction matrices resulting from the diagrams of Figs. 1 and 2 in the angular momentum formalism.

\section{a. Coupling matrices with $2 p 1$ IS IS}

The simplest $2 p 1 h$ coupling matrix is $\mathbf{M}_{r \alpha}^{(\mathrm{Ia})}$, Eq. 34, and it appears already at the $\operatorname{ADC}(2)$ level. Using the definition (B12), we obtain

$$
\begin{aligned}
\mathbf{M}_{\tilde{r} a}^{(\mathrm{Ia})} \equiv & \Delta\left(j_{n_{1}}, j_{n_{2}}, J_{12}\right) \Delta\left(J_{12}, j_{k_{3}}, J_{r}\right)(-1)^{j_{\alpha}+j_{k_{3}}-J_{12}} \frac{\hat{J}_{12}}{\hat{j}_{\alpha}} \\
& \times \sum_{m \leq v} \frac{\mathcal{X}_{m}^{\tilde{n}_{1}} \delta_{m n_{1}}^{(\pi j)} \mathcal{X}_{v}^{\tilde{n}_{2}} \delta_{v n_{2}}^{(\pi j q)}-(-1)^{j_{n_{1}}+j_{n_{2}}-J_{12}} \mathcal{X}_{v}^{\tilde{n}_{1}} \delta_{v n_{1}}^{(\pi j q)} \mathcal{X}_{m}^{\tilde{n}_{2}} \delta_{m n_{2}}^{(\pi j q)}}{\sqrt{1+\delta_{v m}} \sqrt{1+\delta_{\tilde{n}_{1} \tilde{n}_{2}}}} \bar{V}_{m v, a l}^{J_{12}} \sqrt{1+\delta_{a l}} \mathcal{Y}_{l}^{\tilde{k}_{3}} \delta_{l k_{3}}^{(\pi j q)}
\end{aligned}
$$

with the usual "hat" notation

$$
\hat{j} \equiv \sqrt{2 j+1}
$$

and the triangular condition

$$
\Delta\left(j, j^{\prime}, J\right)= \begin{cases}1 & \text { if }\left|j-j^{\prime}\right| \leq J \leq j+j^{\prime}, \\ 0 & \text { otherwise. }\end{cases}
$$

The $\mathbf{M}_{r \alpha}^{(\mathrm{IIa})}$ contribution from Eq. 53 becomes

$$
\begin{aligned}
\mathbf{M}_{\tilde{r} a}^{(\mathrm{IIa})} \equiv \Delta & \Delta\left(j_{n_{1}}, j_{n_{2}}, J_{12}\right) \Delta\left(J_{12}, j_{k_{3}}, J_{r}\right) \frac{(-1)^{j_{\alpha}+j_{k_{3}}-J_{12}}}{\sqrt{1+\delta_{\tilde{n}_{1} \tilde{n}_{2}}}} \frac{\hat{J}_{12}}{\hat{j}_{\alpha}} \sum_{\tilde{k}_{4} \leq \tilde{k}_{5}} \sum_{g \leq d} \Delta\left(j_{k_{4}}, j_{k_{5}}, J_{12}\right) t_{\tilde{k}_{4} \tilde{k}_{5}}^{\tilde{n}_{1} \tilde{n}_{2}, J_{12}} \\
& \times \frac{\left(\mathcal{Y}_{g}^{\tilde{k}_{4}} \delta_{g k_{4}}^{(\pi j q)} \mathcal{Y}_{d}^{\tilde{k}_{5}} \delta_{d k_{5}}^{(\pi j q)}-(-1)^{j_{k_{4}}+j_{k_{5}}-J_{12}} \mathcal{Y}_{d}^{\tilde{k}_{4}} \delta_{d k_{4}}^{(\pi j q)} \mathcal{Y}_{g}^{\tilde{k}_{5}} \delta_{g k_{5}}^{(\pi j q)}\right)^{*}}{\left(1+\delta_{\tilde{k}_{4} \tilde{k}_{5}}\right) \sqrt{1+\delta_{g d}}} \bar{V}_{g d, a l}^{J_{12}} \sqrt{1+\delta_{a l}} \mathcal{Y}_{l}^{\tilde{k}_{3}} \delta_{l k_{3}}^{(\pi j q)},
\end{aligned}
$$


where we have coupled the angular momenta of the $2 p 2 h$ amplitude, Eq. (47), as follows:

$$
\begin{aligned}
t_{\tilde{k}_{3} \tilde{k}_{3}}^{\tilde{n}_{1} \tilde{n}_{2}, J} \equiv & \sum_{\substack{m_{n_{1}} m_{n_{2}} \\
m_{k_{3}} m_{k_{4}}}}\left(j_{n_{1}} j_{n_{2}} m_{n_{1}} m_{n_{2}} \mid J M\right)\left(j_{k_{3}} j_{k_{4}} m_{k_{3}} m_{k_{4}} \mid J-M\right)(-1)^{J-M} t_{k_{3} k_{4}}^{n_{1} n_{2}} \\
= & \sum_{\substack{m \leq v \\
g \leq d}} \frac{\mathcal{X}_{m}^{\tilde{n}_{1}} \delta_{m n_{1}}^{(\pi j q)} \mathcal{X}_{v}^{\tilde{n}_{2}} \delta_{v n_{2}}^{(\pi j q)}-(-1)^{j_{n_{1}}+j_{n_{2}}-J} \mathcal{X}_{v}^{\tilde{n}_{1}} \delta_{v n_{1}}^{(\pi j q)} \mathcal{X}_{m}^{\tilde{n}_{2}} \delta_{m n_{2}}^{(\pi j q)}}{\sqrt{1+\delta_{m v}}} \\
& \quad \times \frac{\bar{V}_{m v, g d}^{J}}{\varepsilon_{\tilde{k}_{4}}^{-}+\varepsilon_{\tilde{k}_{3}}^{-}-\varepsilon_{\tilde{n}_{1}}^{+}-\varepsilon_{\tilde{n}_{2}}^{+}} \frac{\mathcal{Y}_{g}^{\tilde{k}_{3}} \delta_{g k_{3}}^{(\pi j q)} \mathcal{Y}_{d}^{\tilde{k}_{4}} \delta_{d k_{4}}^{(\pi j q)}-(-1)^{j_{k_{3}}+j_{k_{4}}-J} \mathcal{Y}_{d}^{\tilde{k}_{3}} \delta_{d k_{3}}^{(\pi j q)} \mathcal{Y}_{g}^{\tilde{k}_{4}} \delta_{g k_{4}}^{(\pi j q)}}{\sqrt{1+\delta_{r s}}}
\end{aligned}
$$

The $\mathbf{M}_{r \alpha}^{(\mathrm{IIb})}$ of Eq. 54 in the angular momentum coupled representation is given by

$$
\begin{aligned}
& \mathbf{M}_{\tilde{r} a}^{(\mathrm{IIb})} \equiv \Delta\left(j_{n_{1}}, j_{n_{2}}, J_{12}\right) \Delta\left(J_{12}, j_{k_{3}}, J_{r}\right)(-1)^{j_{\alpha}+j_{k_{3}}-J_{12}} \frac{\hat{J}_{12}}{\hat{j}_{\alpha}} \frac{1}{\sqrt{1+\delta_{\tilde{n}_{1} \tilde{n}_{2}}}} \sum_{\tilde{k}_{4} \tilde{n}_{5}} \sum_{m_{l} v} \sum_{J_{2} J_{3}}\left(2 J_{2}+1\right)\left(2 J_{3}+1\right) \\
& \times\left((-1)^{-j_{n_{1}}-j_{n_{2}}+J_{12}}\left\{\begin{array}{lll}
j_{n_{1}} & j_{k_{4}} & J_{2} \\
j_{n_{2}} & J_{3} & j_{n_{5}} \\
J_{12} & j_{k_{3}} & J_{r}
\end{array}\right\} \mathcal{X}_{m}^{\tilde{n}_{1}} \delta_{m n_{1}}^{(\pi j q)} t_{\tilde{k}_{4} \tilde{k}_{3}}^{\tilde{n}_{2} \tilde{n}_{5}, J_{3}}\left(\mathcal{Y}_{v}^{\tilde{k}_{4}} \delta_{v k_{4}}^{(\pi j q)} \mathcal{X}_{l}^{\tilde{n}_{5}} \delta_{l n_{5}}^{(\pi j)}\right)^{*} \sqrt{1+\delta_{g m}} \bar{V}_{v m, a l}^{J_{2}} \sqrt{1+\delta_{a d}}\right. \\
& \left.-\left\{\begin{array}{lll}
j_{n_{2}} & j_{k_{4}} & J_{2} \\
j_{n_{1}} & J_{3} & j_{n_{5}} \\
J_{12} & j_{k_{3}} & J_{r}
\end{array}\right\} \mathcal{X}_{m}^{\tilde{n}_{2}} \delta_{m n_{2}}^{(\pi j q)} t_{\tilde{k}_{4} \tilde{k}_{3}}^{\tilde{n}_{1} \tilde{n}_{5}, J_{3}}\left(\mathcal{Y}_{v}^{\tilde{k}_{4}} \delta_{v k_{4}}^{(\pi j q)} \mathcal{X}_{l}^{\tilde{n}_{5}} \delta_{l n_{5}}^{(\pi j q)}\right)^{*} \sqrt{1+\delta_{g m}} \bar{V}_{v m, a l}^{J_{2}} \sqrt{1+\delta_{a d}}\right),
\end{aligned}
$$

which is explicitly antisymmetrized with respect to the $\tilde{n}_{1}, \tilde{n}_{2}$ indexes.

The coupling matrix $\mathbf{M}_{r \alpha}^{(\mathrm{IIc})}$ of Eq. 57 is implied by the diagram of Fig. $2 \mathrm{c}$. This is the first term that contains an interaction-irreducible $3 \mathrm{NF}$ and it has the following form:

$$
\begin{aligned}
& \mathbf{M}_{\tilde{r} a}^{(\mathrm{IIc})} \equiv \Delta\left(j_{n_{1}}, j_{n_{2}}, J_{12}\right) \Delta\left(J_{12}, j_{k_{3}}, J_{r}\right) \sum_{J_{56} J_{J^{\prime}}} \sum_{\substack{\tilde{k}_{5} \leq \tilde{k}_{6} \\
\tilde{n}_{4}}} \sum_{\substack{l \leq m \\
l}} \Delta\left(j_{k_{5}}, j_{k_{6}}, J_{56}\right) \Delta\left(j_{n_{4}}, J_{56}, j_{\alpha}\right) \frac{(-1)^{j_{n_{4}}+j_{\alpha}-J_{56}+2 J^{\prime}}}{\sqrt{1+\delta_{\tilde{n}_{1} \tilde{n}_{2}}}} \frac{\hat{J}_{56}}{\hat{j}_{\alpha}}\left(2 J^{\prime}+1\right) \\
& \times\left\{\begin{array}{l}
j_{k_{3}} J_{12} j_{\alpha} \\
j_{n_{4}} J_{56} J^{\prime}
\end{array}\right\} t_{\tilde{k}_{5} \tilde{k}_{6} \tilde{k}_{3}, J_{56}}^{\tilde{n}_{2} \tilde{n}_{2} \tilde{n}_{4}, J_{12} J^{\prime}} \frac{\left(\mathcal{Y}_{m}^{\tilde{k}_{5}} \delta_{n k_{5}}^{(\pi j q)} \mathcal{Y}_{v}^{\tilde{k}_{6}} \delta_{v k_{6}}^{(\pi j q)}-(-1)^{j_{k_{5}}+j_{k_{6}}-J_{56}} \mathcal{Y}_{v}^{\tilde{k}_{5}} \delta_{v k_{5}}^{(\pi j q)} \mathcal{Y}_{m}^{\tilde{k}_{6}} \delta_{m k_{6}}^{(\pi j q)}\right)^{*}}{\left(1+\delta_{\tilde{k}_{5} \tilde{k}_{6}}\right) \sqrt{1+\delta_{m v}}} \\
& \times\left(\mathcal{X}_{l}^{\tilde{n}_{4}} \delta_{l n_{4}}^{(\pi j q)}\right)^{*} \bar{V}_{m v, a l}^{J_{56}} \sqrt{1+\delta_{a l}},
\end{aligned}
$$

which is expressed in terms of the $3 p 3 h$ amplitude Eq. 48). In the angular momentum form, this is

$$
\begin{gathered}
t_{\tilde{k}_{5} \tilde{k}_{6} \tilde{k}_{3}, J_{56}}^{\tilde{n}_{1} \tilde{n}_{2} \tilde{n}_{4}, J_{12} J^{\prime}} \equiv \sum_{\substack{g \leq d \\
t \leq p}} \frac{\mathcal{X}_{g}^{\tilde{n}_{1}} \delta_{g n_{1}}^{(\pi j q)} \mathcal{X}_{d}^{\tilde{n}_{2}} \delta_{d n_{2}}^{(\pi j q)}-(-1)^{j_{n_{1}}+j_{n_{2}}-J_{12}} \mathcal{X}_{d}^{\tilde{n}_{1}} \delta_{d n_{1}}^{(\pi j q)} \mathcal{X}_{g}^{\tilde{n}_{2}} \delta_{g n_{2}}^{(\pi j q)}}{\left(1+\delta_{g d}\right)} \frac{\mathcal{X}_{r}^{\tilde{n}_{4}} \delta_{r n_{4}}^{(\pi j q)} W_{g d r, t p s}^{J_{12} J_{56} J^{\prime}} \mathcal{Y}_{s}^{\tilde{k}_{3}} \delta_{s k_{3}}^{(\pi j q)}}{\varepsilon_{\tilde{k}_{3}}^{-}+\varepsilon_{\tilde{k}_{5}}^{-}+\varepsilon_{\tilde{k}_{6}}^{-}-\varepsilon_{\tilde{n}_{1}}^{+}-\varepsilon_{\tilde{n}_{2}}^{+}-\varepsilon_{\tilde{n}_{4}}^{+}} \\
\times \frac{\mathcal{Y}_{t}^{\tilde{k}_{5}} \delta_{t k_{5}}^{(\pi j q)} \mathcal{Y}_{p}^{\tilde{k}_{6}} \delta_{p k_{6}}^{(\pi j q)}-(-1)^{j_{k_{5}}+j_{k_{6}}-J_{56}} \mathcal{Y}_{p}^{\tilde{k}_{5}} \delta_{p k_{5}}^{(\pi j q)} \mathcal{Y}_{t}^{\tilde{k}_{6}} \delta_{t k_{6}}^{(\pi j q)}}{\left(1+\delta_{t p}\right)}
\end{gathered}
$$

\section{b. Coupling matrices with $2 h 1 p$ ISCs}

The backward-in-time contributions to the self-energy involve ISCs with $2 h 1 p$. The angular momentum representation for the $\mathrm{ADC}(2)$ coupling matrix, Eq. (35), is:

$$
\begin{aligned}
\mathbf{N}_{a \tilde{s}}^{(\mathrm{Ia})} \equiv & \Delta\left(j_{k_{1}}, j_{k_{2}}, J_{12}\right) \Delta\left(J_{12}, j_{n_{3}}, J_{s}\right)(-1)^{j_{\alpha}-m_{\alpha}}(-1)^{j_{\alpha}-j_{n_{3}}-J_{12}} \frac{\hat{J}_{12}}{\hat{j}_{\alpha}} \\
& \times \sum_{m \leq v} \sqrt{1+\delta_{a l}} \bar{V}_{a l, m v}^{J_{12}} \frac{\mathcal{Y}_{m}^{\tilde{k}_{1}} \delta_{m k_{1}}^{(\pi j q)} \mathcal{Y}_{v}^{\tilde{k}_{2}} \delta_{v k_{2}}^{(\pi j q)}-(-1)^{j_{k_{1}}+j_{k_{2}}-J_{12}} \mathcal{Y}_{v}^{\tilde{k}_{1}} \delta_{v k_{1}}^{(\pi j q)} \mathcal{Y}_{m}^{\tilde{k}_{2}} \delta_{m k_{2}}^{(\pi j q)}}{\sqrt{1+\delta_{v m}} \sqrt{1+\delta_{\tilde{k}_{1} \tilde{k}_{2}}}} \mathcal{X}_{l}^{\tilde{n}_{3}} \delta_{l n_{3}}^{(\pi j q)}
\end{aligned}
$$


The $\mathrm{ADC}(3)$ ladder term coupling matrix of Eq. 55 becomes

$$
\begin{gathered}
\mathbf{N}_{a \tilde{s}}^{(\mathrm{IIa})} \equiv \Delta\left(j_{k_{1}}, j_{k_{2}}, J_{12}\right) \Delta\left(J_{12}, j_{n_{3}}, J_{s}\right)(-1)^{j_{\alpha}-m_{\alpha}} \frac{(-1)^{j_{\alpha}-j_{n_{3}}-J_{12}}}{\sqrt{1+\delta_{\tilde{k}_{1} \tilde{k}_{2}}}} \frac{\hat{J}_{12}}{\hat{j}_{\alpha}} \sum_{\tilde{n}_{4} \leq \tilde{n}_{5}} \sum_{g \leq d} \Delta\left(j_{n_{4}}, j_{n_{5}}, J_{1}\right) \sqrt{1+\delta_{a l}} \bar{V}_{a l, g d}^{J_{12}} \\
\quad \times \mathcal{X}_{l}^{\tilde{n}_{3}} \delta_{l n_{3}}^{(\pi j q)} \frac{\left(\mathcal{X}_{g}^{\tilde{n}_{4}} \delta_{g n_{4}}^{(\pi j q)} \mathcal{X}_{d}^{\tilde{n}_{5}} \delta_{d n_{5}}^{(\pi j q)}-(-1)^{j_{\gamma}+j_{\delta}-J_{12}} \mathcal{X}_{d}^{\tilde{n}_{4}} \delta_{d n_{4}}^{(\pi j q)} \mathcal{X}_{g}^{\tilde{n}_{5}} \delta_{g n_{5}}^{(\pi j q)}\right)^{*}}{\sqrt{1+\delta_{g d}}\left(1+\delta_{\tilde{n}_{4} \tilde{n}_{5}}\right)} t_{\tilde{k}_{1} \tilde{n}_{2} \tilde{n}_{5}, J_{12}}^{\tilde{n}_{12}}
\end{gathered}
$$

and the corresponding particle-hole channel, Eq. [56], is

$$
\begin{aligned}
& \mathbf{N}_{a \tilde{s}}^{(\mathrm{IIb})} \equiv \Delta\left(j_{k_{1}}, j_{k_{2}}, J_{12}\right) \Delta\left(J_{12}, j_{n_{3}}, J_{s}\right)(-1)^{j_{\alpha}-m_{\alpha}} \frac{(-1)^{j_{\alpha}-j_{n_{3}}-J_{12}}}{\sqrt{1+\delta_{\tilde{k}_{1} \tilde{k}_{2}}}} \frac{\hat{J}_{12}}{\hat{j}_{\alpha}} \sum_{\tilde{n}_{4} \tilde{k}_{5}} \sum_{m_{l} v} \sum_{J_{2} J_{3}}\left(2 J_{2}+1\right)\left(2 J_{3}+1\right) \\
& \times\left((-1)^{-j_{k_{1}}-j_{k_{2}}+J_{12}}\left\{\begin{array}{lll}
j_{k_{1}} & j_{n_{4}} & J_{2} \\
j_{k_{2}} & J_{3} & j_{k_{5}} \\
J_{12} & j_{n_{3}} & J_{s}
\end{array}\right\} \sqrt{1+\delta_{a l}} \bar{V}_{a l, v m}^{J_{2}} \sqrt{1+\delta_{v m}}\left(\mathcal{X}_{v}^{\tilde{n}_{4}} \delta_{v n_{4}}^{(\pi j q)} \mathcal{Y}_{l}^{\tilde{k}_{5}} \delta_{l k_{5}}^{(\pi j q)}\right)^{*} \mathcal{Y}_{m}^{\tilde{k}_{1}} \delta_{m k_{1}}^{(\pi j q)} t_{\tilde{k}_{2} \tilde{k}_{5}}^{\tilde{n}_{4} \tilde{n}_{3}, J_{3}}\right. \\
& \left.-\left\{\begin{array}{lll}
j_{k_{2}} & j_{n_{4}} & J_{2} \\
j_{k_{1}} & J_{3} & j_{k_{5}} \\
J_{12} & j_{n_{3}} & J_{s}
\end{array}\right\} \sqrt{1+\delta_{a l}} \bar{V}_{a l, v m}^{J_{2}} \sqrt{1+\delta_{v m}}\left(\mathcal{X}_{v}^{\tilde{n}_{4}} \delta_{v n_{4}}^{(\pi j q)} \mathcal{Y}_{l}^{\tilde{k}_{5}} \delta_{l k_{5}}^{(\pi j q)}\right)^{*} \mathcal{Y}_{m}^{\tilde{k}_{2}} \delta_{m k_{2}}^{(\pi j q)} t_{\tilde{k}_{1} \tilde{k}_{5}}^{\tilde{n}_{4} \tilde{n}_{3}, J_{3}}\right),
\end{aligned}
$$

which is explicitly antisymmetrized with respect to the $\tilde{k}_{1}, \tilde{k}_{2}$ indices.

Finally, the coupling matrix $\mathbf{N}_{\alpha s}^{(\mathrm{IIc})}$ of Eq. $\sqrt{58}$ is found in the backward-in-time diagram of Fig. 2c and contains a $3 \mathrm{NF}$. It has the following form in the angular momentum coupling representation:

$$
\begin{aligned}
\mathbf{N}_{a \tilde{s}}^{(\mathrm{IIc})} \equiv \Delta & \left(j_{k_{1}}, j_{k_{2}}, J_{12}\right) \Delta\left(J_{12}, j_{n_{3}}, J_{s}\right) \sum_{J_{45} J^{\prime}} \sum_{\tilde{n}_{4} \leq \tilde{n}_{5}} \sum_{v \leq m} \Delta\left(j_{n_{5}}, j_{n_{6}}, J_{56}\right) \Delta\left(J_{56}, j_{k_{4}}, j_{\alpha}\right) \\
& \times(-1)^{j_{\alpha}-m_{\alpha}} \frac{(-1)^{j_{\alpha}+2 j_{n_{3}}+j_{k_{4}}-J_{56}+2 J^{\prime}}}{\sqrt{1+\delta_{\tilde{k}_{1} \tilde{k}_{2}}}} \frac{\hat{J}_{12}}{\hat{j}_{\alpha}}\left(2 J^{\prime}+1\right)\left\{\begin{array}{c}
j_{n_{3}} J_{45} J^{\prime} \\
j_{k_{4}} J_{12} j_{\alpha}
\end{array}\right\} \sqrt{1+\delta_{a l}} \bar{V}_{a l, m v}^{J_{56}} \\
& \times \frac{\left(\mathcal{X}_{m}^{\tilde{n}_{5}} \delta_{m n_{5}}^{(\pi j q)} \mathcal{X}_{v}^{\tilde{n}_{6}} \delta_{v n_{6}}^{(\pi j q)}-(-1)^{j_{n_{5}}+j_{n_{6}}-J_{56}} \mathcal{X}_{v}^{\tilde{n}_{5}} \delta_{v n_{5}}^{(\pi j q)} \mathcal{X}_{m}^{\tilde{n}_{6}} \delta_{m n_{6}}^{(\pi j q)}\right.}{\left(1+\delta_{\tilde{n}_{5} \tilde{n}_{6}}\right) \sqrt{1+\delta_{m v}}}\left(\mathcal{Y}_{l}^{\tilde{k}_{4}} \delta_{l k_{4}}^{(\pi j q)}\right)^{*} t_{\tilde{k}_{1} \tilde{k}_{5} \tilde{k}_{2} \tilde{k}_{4}, \tilde{n}_{32}, J_{56} J^{\prime}}^{\tilde{n}_{12}} .
\end{aligned}
$$

\section{c. Interaction matrices with $2 p 1 h$ and $2 h 1 p$ ISCs}

The interaction matrix $\mathbf{C}_{\tilde{r} \tilde{r}^{\prime}}$ can connect $2 p 1 h$ propagators through particle-particle, particle-hole and $3 \mathrm{NFs}$, according to the terms

$$
\mathbf{C}_{\tilde{r} \tilde{r}^{\prime}} \equiv \mathbf{C}_{\tilde{r} \tilde{r}^{\prime}}^{p p}+\mathbf{C}_{\tilde{r} \tilde{r}^{\prime}}^{p h}+\mathbf{C}_{\tilde{r} \tilde{r}^{\prime}}^{3 N},
$$

which have been introduced in Eqs. (59), 60), and (63), respectively.

The particle-particle interaction matrix results from the diagram in Fig. 2a. Using the coupling convention of Eq. (B14, we have:

$$
\begin{aligned}
& \mathbf{C}_{\tilde{r} \tilde{r}^{\prime}}^{p p} \equiv \Delta\left(j_{n_{1}}, j_{n_{2}}, J_{12}\right) \Delta\left(J_{12}, j_{k_{3}}, J_{r}\right) \Delta\left(j_{n_{4}}, j_{n_{5}}, J_{12}\right) \Delta\left(J_{12}, j_{k_{6}}, J_{r}\right) \\
& \times \delta_{J_{12} J_{45}} \delta_{\tilde{k}_{3} \tilde{k}_{6}} \sum_{\substack{m \leq v \\
l \leq p}} \frac{\mathcal{X}_{m}^{\tilde{n}_{1}} \delta_{m n_{1}}^{(\pi j q)} \mathcal{X}_{v}^{\tilde{n}_{2}} \delta_{v n_{2}}^{(\pi j q)}-(-1)^{j_{n_{1}}+j_{n_{2}}-J_{12}} \mathcal{X}_{v}^{\tilde{n}_{1}} \delta_{v n_{1}}^{(\pi j q)} \mathcal{X}_{m}^{\tilde{n}_{2}} \delta_{m n_{2}}^{(\pi j q)}}{\sqrt{1+\delta_{\tilde{n}_{1} \tilde{n}_{2}}} \sqrt{1+\delta_{m v}}} \\
& \times \bar{V}_{m v, l p}^{J_{12}} \frac{\left(\mathcal{X}_{l}^{\tilde{n}_{4}} \delta_{l n_{4}}^{(\pi j q)} \mathcal{X}_{p}^{\tilde{n}_{5}} \delta_{p n_{5}}^{(\pi j q)}-(-1)^{j_{n_{4}}+j_{n_{5}}-J_{12}} \mathcal{X}_{p}^{\tilde{n}_{4}} \delta_{p n_{4}}^{(\pi j q)} \mathcal{X}_{l}^{\tilde{n}_{5}} \delta_{l n_{5}}^{(\pi j q)}\right)^{*}}{\sqrt{1+\delta_{l p} \sqrt{1+\delta_{\tilde{n}_{4} \tilde{n}_{5}}}}} .
\end{aligned}
$$


The particle-hole $\mathbf{C}_{\tilde{r} \tilde{r}^{\prime}}^{p h}$ comes from the ring diagram in Fig. 2b. which contains four terms owing to the antisymmetrization specified in Eq. (60),

$$
\begin{aligned}
& \mathbf{C}_{\tilde{r} \tilde{r}^{\prime}}^{p h} \equiv \Delta\left(j_{n_{1}}, j_{n_{2}}, J_{12}\right) \Delta\left(J_{12}, j_{k_{3}}, J_{r}\right) \Delta\left(j_{n_{4}}, j_{n_{5}}, J_{45}\right) \Delta\left(J_{45}, j_{k_{6}}, J_{r}\right) \frac{1}{2} \sum_{\substack{m v \\
p l}} \sum_{J} \frac{\hat{J}_{12} \hat{J}_{45}(2 J+1)}{\sqrt{1+\delta_{\tilde{n}_{1} \tilde{n}_{2}}} \sqrt{1+\delta_{\tilde{n}_{4} \tilde{n}_{5}}}} \\
& \times\left(\left\{\begin{array}{ccc}
j_{n_{1}} & j_{n_{2}} & J_{12} \\
j_{n_{5}} & J & j_{k_{3}} \\
J_{45} & j_{k_{6}} & J_{r}
\end{array}\right\} \mathcal{X}_{v}^{\tilde{n}_{2}} \delta_{v n_{2}}^{(\pi j q)} \mathcal{Y}_{p}^{\tilde{k}_{6}} \delta_{p k_{6}}^{(\pi j q)} \sqrt{1+\delta_{m v}} V_{m v, p l}^{J} \sqrt{1+\delta_{p l}}\left(\mathcal{X}_{l}^{\tilde{n}_{5}} \delta_{l n_{5}}^{(\pi j q)} \mathcal{Y}_{m}^{\tilde{k}_{3}} \delta_{m k_{3}}^{(\pi j q)}\right)^{*} \delta_{\tilde{n}_{1} \tilde{n}_{4}}\right. \\
& -(-1)^{j_{n_{1}}+j_{n_{2}}-J_{12}}\left\{\begin{array}{ccc}
j_{n_{2}} & j_{n_{1}} & J_{12} \\
j_{n_{5}} & J & j_{k_{3}} \\
J_{45} & j_{k_{6}} & J_{r}
\end{array}\right\} \mathcal{X}_{v}^{\tilde{n}_{1}} \delta_{v n_{1}}^{(\pi j q)} \mathcal{Y}_{p}^{\tilde{k}_{6}} \delta_{p k_{6}}^{(\pi j q)} \sqrt{1+\delta_{m v}} V_{m v, p l}^{J} \sqrt{1+\delta_{p l}}\left(\mathcal{X}_{l}^{\tilde{n}_{5}} \delta_{l n_{5}}^{(\pi j q)} \mathcal{Y}_{m}^{\tilde{k}_{3}} \delta_{m k_{3}}^{(\pi j q)}\right)^{*} \delta_{\tilde{n}_{2} \tilde{n}_{4}} \\
& -(-1)^{j_{n_{4}}+j_{n_{5}}-J_{45}}\left\{\begin{array}{ccc}
j_{n_{1}} & j_{n_{2}} & J_{12} \\
j_{n_{4}} & J & j_{k_{3}} \\
J_{45} & j_{k_{6}} & J_{r}
\end{array}\right\} \mathcal{X}_{v}^{\tilde{n}_{2}} \delta_{v n_{2}}^{(\pi j q)} \mathcal{Y}_{p}^{\tilde{k}_{6}} \delta_{p k_{6}}^{(\pi j q)} \sqrt{1+\delta_{m v}} V_{m v, p l}^{J} \sqrt{1+\delta_{p l}}\left(\mathcal{X}_{l}^{\tilde{n}_{4}} \delta_{l n_{4}}^{(\pi j q)} \mathcal{Y}_{m}^{\tilde{k}_{3}} \delta_{m k_{3}}^{(\pi j q)}\right)^{*} \delta_{\tilde{n}_{1} \tilde{n}_{5}} \\
& +(-1)^{j_{n_{1}}+j_{n_{2}}-J_{12}}(-1)^{j_{n_{4}}+j_{n_{5}}-J_{45}} \\
& \left.\times\left\{\begin{array}{ccc}
j_{n_{2}} & j_{n_{1}} & J_{12} \\
j_{n_{4}} & J & j_{k_{3}} \\
J_{45} & j_{k_{6}} & J_{r}
\end{array}\right\} \mathcal{X}_{v}^{\tilde{n}_{1}} \delta_{v n_{1}}^{(\pi j q)} \mathcal{Y}_{p}^{\tilde{k}_{6}} \delta_{p k_{6}}^{(\pi j q)} \sqrt{1+\delta_{m v}} V_{m v, p l}^{J} \sqrt{1+\delta_{p l}}\left(\mathcal{X}_{l}^{\tilde{n}_{4}} \delta_{l n_{4}}^{(\pi j q)} \mathcal{Y}_{m}^{\tilde{k}_{3}} \delta_{m k_{3}}^{(\pi j q)}\right)^{*} \delta_{\tilde{n}_{2} \tilde{n}_{5}}\right) .
\end{aligned}
$$

The 3 NF interaction matrix in Eq. B30 reads,

$$
\begin{aligned}
\mathbf{C}_{\tilde{r} \tilde{r}^{\prime}}^{3 N} \equiv & \Delta\left(j_{n_{1}}, j_{n_{2}}, J_{12}\right) \Delta\left(J_{12}, j_{k_{3}}, J_{r}\right) \Delta\left(j_{n_{4}}, j_{n_{5}}, J_{45}\right) \Delta\left(J_{45}, j_{k_{6}}, J_{r}\right) \\
& \times \sum_{m \leq v} \sum_{g \leq d} \frac{\mathcal{X}_{m}^{\tilde{n}_{1}} \delta_{m n_{1}}^{(\pi j q)} \mathcal{X}_{v}^{\tilde{n}_{2}} \delta_{v n_{2}}^{(\pi j q)}-(-1)^{j_{n_{1}}+j_{n_{2}}-J_{12}} \mathcal{X}_{v}^{\tilde{n}_{1}} \delta_{v n_{1}}^{(\pi j q)} \mathcal{X}_{m}^{\tilde{n}_{2}} \delta_{m n_{2}}^{(\pi j q)}}{\sqrt{1+\delta_{\tilde{n}_{1} \tilde{n}_{2}}}\left(1+\delta_{m v}\right)} \mathcal{Y}_{l}^{\tilde{k}_{3}} \delta_{l k_{3}}^{(\pi j q)} \\
& \times W_{m v l^{-1}, g d p^{-1}}^{(p p h) J_{12} J_{45} J^{\prime}} \frac{\left(\mathcal{X}_{g}^{\tilde{n}_{4}} \delta_{g n_{4}}^{(\pi j q)} \mathcal{X}_{d}^{\tilde{n}_{5}} \delta_{d n_{5}}^{(\pi j q)}-(-1)^{j_{n_{4}}+j_{n_{5}}-J_{45}} \mathcal{X}_{d}^{\tilde{n}_{4}} \delta_{d n_{4}}^{(\pi j q)} \mathcal{X}_{g}^{\tilde{n}_{5}} \delta_{g n_{5}}^{(\pi j q)}\right)^{*}}{\left(1+\delta_{g d}\right) \sqrt{1+\delta_{\tilde{n}_{4} \tilde{n}_{5}}}}\left(\mathcal{Y}_{p}^{\tilde{k}_{6}} \delta_{p k_{6}}^{(\pi j q)}\right)^{*}
\end{aligned}
$$

where we have the $3 \mathrm{NF}$ coupled in the $p p h$ channel from Eq. B10.

The backward-in-time interaction matrix $\mathbf{D}_{\tilde{s} \tilde{s}^{\prime}}$ can connect the $2 h 1 p$ propagators through hole-hole, hole-particle and backward-in-time $3 \mathrm{NFs}$, according to,

$$
\mathbf{D}_{\tilde{s} \tilde{s}^{\prime}} \equiv \mathbf{D}_{\tilde{s} \tilde{s}^{\prime}}^{h h}+\mathbf{D}_{\tilde{s} \tilde{s}^{\prime}}^{h p}+\mathbf{D}_{\tilde{s} \tilde{s}^{\prime}}^{3 N}
$$

with the three matrices on the right-hand side introduced in Eqs. [61), 62), and (64), respectively.

The hole-hole interaction matrix resulting from the diagram in Fig. $2 \mathrm{a}$ reads,

$$
\begin{aligned}
\mathbf{D}_{\tilde{s} \tilde{s}^{\prime}}^{h h} \equiv-\Delta\left(j_{k_{1}}, j_{k_{2}}, J_{12}\right) \Delta\left(J_{12}, j_{n_{3}}, J_{s}\right) \Delta\left(j_{k_{4}}, j_{k_{5}}, J_{12}\right) \Delta\left(J_{12}, j_{n_{6}}, J_{s}\right) \\
\times \delta_{J_{12} J_{45}} \delta_{\tilde{n}_{3} \tilde{n}_{6}} \sum_{\substack{m \leq v \\
l \leq p}} \frac{\left(\mathcal{Y}_{m}^{\tilde{k}_{1}} \delta_{m k_{1}}^{(\pi j q)} \mathcal{Y}_{v}^{\tilde{k}_{2}} \delta_{v k_{2}}^{(\pi j q)}-(-1)^{j_{k_{1}}+j_{k_{2}}-J_{12}} \mathcal{Y}_{v}^{\tilde{k}_{1}} \delta_{v k_{1}}^{(\pi j q)} \mathcal{Y}_{m}^{\tilde{k}_{2}} \delta_{n k_{2}}^{(\pi j q)}\right)^{*}}{\sqrt{1+\delta_{g d}} \sqrt{1+\delta_{\tilde{k}_{1} \tilde{k}_{2}}}} \bar{V}_{m v, l p}^{J_{12}} \\
\times \frac{\mathcal{Y}_{l}^{\tilde{k}_{4}} \delta_{l k_{4}}^{(\pi j q)} \mathcal{Y}_{p}^{\tilde{k}_{5}} \delta_{p k_{5}}^{(\pi j q)}-(-1)^{j_{k_{4}}+j_{k_{5}}-J_{12}} \mathcal{Y}_{p}^{\tilde{k}_{4}} \delta_{p k_{4}}^{(\pi j q)} \mathcal{Y}_{l}^{\tilde{k}_{5}} \delta_{l k_{5}}^{(\pi j q)}}{\sqrt{1+\delta_{t p}} \sqrt{1+\delta_{\tilde{k}_{4} \tilde{k}_{5}}}}
\end{aligned}
$$

while $\mathbf{D}_{\tilde{s} \tilde{s}^{\prime}}^{h p}$ results from the ring diagram in Fig. $2 \mathrm{~b}$ and contains four different terms owing to the antisymmetrization 
specified in Eq. 62]:

$$
\begin{aligned}
& \mathbf{D}_{\tilde{s} \tilde{s}^{\prime}}^{h p} \equiv \Delta\left(j_{k_{1}}, j_{k_{2}}, J_{12}\right) \Delta\left(J_{12}, j_{n_{3}}, J_{s}\right) \Delta\left(j_{k_{4}}, j_{k_{5}}, J_{45}\right) \Delta\left(J_{45}, j_{n_{6}}, J_{s}\right) \frac{1}{2} \sum_{\substack{g d \\
t p}} \sum_{J}(-1)^{2 j_{k_{6}}-2 j_{k_{3}}} \frac{\hat{J}_{12} \hat{J}_{45}(2 J+1)}{\sqrt{1+\delta_{\tilde{k}_{1} \tilde{k}_{2}}} \sqrt{1+\delta_{\tilde{k}_{4} \tilde{k}_{5}}}} \\
& \times\left(\left\{\begin{array}{ccc}
j_{k_{1}} & j_{k_{2}} & J_{12} \\
j_{k_{5}} & J & j_{n_{3}} \\
J_{45} & j_{n_{6}} & J_{s}
\end{array}\right\}\left(\mathcal{Y}_{m}^{\tilde{k}_{2}} \delta_{m k_{2}}^{(\pi j q)} \mathcal{X}_{p}^{\tilde{n}_{3}} \delta_{p n_{3}}^{(\pi j q)}\right)^{*} \sqrt{1+\delta_{m v}} V_{v m, p l}^{J} \sqrt{1+\delta_{p l}} \mathcal{Y}_{l}^{\tilde{k}_{5}} \delta_{l k_{5}}^{(\pi j q)} \mathcal{X}_{v}^{\tilde{n}_{6}} \delta_{v n_{6}}^{(\pi j q)} \delta_{\tilde{k}_{1} \tilde{k}_{4}}\right. \\
& -(-1)^{j_{k_{1}}+j_{k_{2}}-J_{12}}\left\{\begin{array}{lll}
j_{k_{2}} & j_{k_{1}} & J_{12} \\
j_{k_{5}} & J & j_{n_{3}} \\
J_{45} & j_{n_{6}} & J_{s}
\end{array}\right\}\left(\mathcal{Y}_{m}^{\tilde{k}_{1}} \delta_{m k_{1}}^{(\pi j q)} \mathcal{X}_{p}^{\tilde{n}_{3}} \delta_{p n_{3}}^{(\pi j q)}\right)^{*} \sqrt{1+\delta_{m v}} V_{v m, p l}^{J} \sqrt{1+\delta_{p l}} \mathcal{Y}_{l}^{\tilde{k}_{5}} \delta_{l k_{5}}^{(\pi j q)} \mathcal{X}_{v}^{\tilde{n}_{6}} \delta_{v n_{6}}^{(\pi j q)} \delta_{\tilde{k}_{2} \tilde{k}_{4}} \\
& -(-1)^{j_{k_{4}}+j_{k_{5}}-J_{45}}\left\{\begin{array}{lll}
j_{k_{1}} & j_{k_{2}} & J_{12} \\
j_{k_{4}} & J & j_{n_{3}} \\
J_{45} & j_{n_{6}} & J_{s}
\end{array}\right\}\left(\mathcal{Y}_{m}^{\tilde{k}_{2}} \delta_{m k_{2}}^{(\pi j q)} \mathcal{X}_{p}^{\tilde{n}_{3}} \delta_{p n_{3}}^{(\pi j q)}\right)^{*} \sqrt{1+\delta_{m v}} V_{v m, p l}^{J} \sqrt{1+\delta_{p l}} \mathcal{Y}_{l}^{\tilde{k}_{4}} \delta_{l k_{4}}^{(\pi j q)} \mathcal{X}_{v}^{\tilde{n}_{6}} \delta_{v n_{6}}^{(\pi j q)} \delta_{\tilde{k}_{1} \tilde{k}_{5}} \\
& +(-1)^{j_{k_{1}}+j_{k_{2}}-J_{12}}(-1)^{j_{k_{4}}+j_{k_{5}}-J_{45}} \\
& \left.\times\left\{\begin{array}{ccc}
j_{k_{2}} & j_{k_{1}} & J_{12} \\
j_{k_{4}} & J & j_{n_{3}} \\
J_{45} & j_{n_{6}} & J_{s}
\end{array}\right\}\left(\mathcal{Y}_{m}^{\tilde{k}_{1}} \delta_{m k_{1}}^{(\pi j q)} \mathcal{X}_{p}^{\tilde{n}_{3}} \delta_{p n_{3}}^{(\pi j q)}\right)^{*} \sqrt{1+\delta_{m v}} V_{v m, p l}^{J} \sqrt{1+\delta_{p l}} \mathcal{Y}_{l}^{\tilde{k}_{4}} \delta_{l k_{4}}^{(\pi j q)} \mathcal{X}_{v}^{\tilde{n}_{6}} \delta_{v n_{6}}^{(\pi j q)} \delta_{\tilde{k}_{2} \tilde{k}_{5}}\right) .
\end{aligned}
$$

Finally, the backward-in-time 3NF interaction matrix in Eq. (B34) is given by

$$
\begin{aligned}
\mathbf{D}_{\tilde{s} \tilde{s}^{\prime}}^{3 N} \equiv & -\Delta\left(j_{k_{1}}, j_{k_{2}}, J_{12}\right) \Delta\left(J_{12}, j_{n_{3}}, J_{s}\right) \Delta\left(j_{k_{4}}, j_{k_{5}}, J_{45}\right) \Delta\left(J_{45}, j_{n_{6}}, J_{s}\right) \\
& \times \sum_{m \leq v} \sum_{g \leq d} \frac{\left(\mathcal{Y}_{m}^{\tilde{k}_{1}} \delta_{m k_{1}}^{(\pi j q)} \mathcal{Y}_{v}^{\tilde{k}_{2}} \delta_{v k_{2}}^{(\pi j q)}-(-1)^{j_{k_{1}}+j_{k_{2}}-J_{12}} \mathcal{Y}_{v}^{\tilde{k}_{1}} \delta_{v k_{1}}^{(\pi j q)} \mathcal{Y}_{m}^{\tilde{k}_{2}} \delta_{m k_{2}}^{(\pi j q)}\right)^{*}}{\sqrt{1+\delta_{\tilde{k}_{1} \tilde{k}_{2}}}\left(1+\delta_{g d}\right)}\left(\mathcal{X}_{l}^{\tilde{n}_{3}} \delta_{l n_{3}}^{(\pi j q)}\right)^{*} \\
& \times W_{m v l^{-1}, g d p^{-1}}^{(p p h) J_{12} J_{45} J^{\prime}} \frac{\mathcal{Y}_{g}^{\tilde{k}_{4}} \delta_{g k_{4}}^{(\pi j q)} \mathcal{Y}_{d}^{\tilde{k}_{5}} \delta_{d k_{5}}^{(\pi j q)}-(-1)^{j_{k_{4}}+j_{k_{5}}-J_{45}} \mathcal{Y}_{d}^{\tilde{k}_{4}} \delta_{g k_{4}}^{(\pi j q)} \mathcal{Y}_{g}^{\tilde{k}_{5}} \delta_{g k_{5}}^{(\pi j q)} \mathcal{X}_{p}^{\tilde{n}_{6}} \delta_{p n_{6}}^{(\pi j q)} .}{\sqrt{1+\delta_{\tilde{k}_{4} \tilde{k}_{5}}}\left(1+\delta_{t p}\right)} .
\end{aligned}
$$

\section{Appendix C: Self-energy without renormalization of the propagators}

This Appendix discusses how the $\mathrm{ADC}(3)$ equations need to be modified when one releases the assumption of full self-consistency. This is the case for most of applications in quantum chemistry and also for state-of-theart nuclear structure studies, where fully dressed propagators become too complex to be able to expand the dynamic self-energy $\widetilde{\Sigma}_{\alpha \beta}(\omega)$. In the latter case, one is forced to implement self-consistency only at the level of the static self-energy, Eq. (18), while Eqs. (A1)- (A4) that generate the dynamic part are based on an uncorrelated (bare) propagator (this is the so-called ' $s c 0$ ' approximation introduced and discussed in Ref. [35]). Without selfconsistency, one needs to follow the standard perturbation approach and to expand the self-energy in terms of reference mean-field propagators. This means that nonskeleton diagrams also need to be added to the perturbative expansion that is used to constrain the $\operatorname{ADC}(n)$ interaction and coupling matrices. For calculations up to order $n=3$, there are substantially two consequences. First, Eq. 14 for the static self-energy must be reexpressed in terms of the reference propagator as shown in Appendix C1. One does this by expanding both the (correlated) $1 \mathrm{~B}$ and $2 \mathrm{~B}$ density matrices, $\rho_{\alpha \beta}$ and $\Gamma_{\alpha \beta, \gamma \delta}$, with the inclusion of nonskeleton terms. The three terms of Eq. (14) still generate the skeleton diagrams of Fig. 9 but now there are 16 additional higher order contributions in terms of the effective forces $\widetilde{U}$ and $\widetilde{V}$, as shown in Figs. 12 and 14 below. Note that a few of these diagrams are of skeleton type and they should be included also when $\widetilde{U}$ is calculated self-consistently. They result from the skeleton expansion of the 2B Green's function and density matrix (see Eqs. (14) and (17)) and will be identified further below. Second, the dynamic self-energy receives four third-order nonskeleton diagrams that are obtained by inserting the (first-order term of the) 1B operator into the uncorrelated fermionic lines that form the diagrams of the dynamic self-energy at second order. These are derived in Section $\mathrm{C} 2$ and they generate additional contributions to the $\mathrm{ADC}(3)$ equations. It is useful 


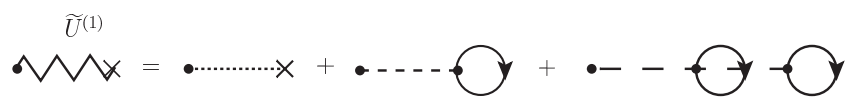

FIG. 9. Diagrammatic representation of the first-order part $\widetilde{U}^{(1)}$ (zigzag line) of the effective 1B interaction of Eq. 13 . Fermionic lines here denote uncorrelated propagators. Dotted lines denote the $1 \mathrm{~B}$ potential $U_{\alpha \beta}$, while short (long) dashed lines denote $2 \mathrm{~N}(3 \mathrm{~N})$ interactions. This is the fist term of the expansion of Eq. $\mathrm{C} 2$ and it is given in full by Eq. (C4).

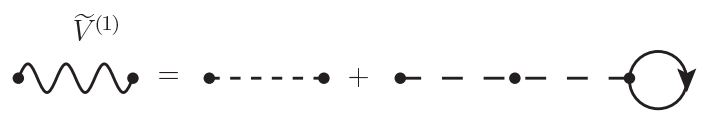

FIG. 10. Diagrammatic representation of $\widetilde{V}^{(1)}$ given by Eq. C5. This is the first-order term in the expansion of Eq. (C3) of the effective $2 \mathrm{~N}$ interaction.

to note that these additional terms cancel exactly when one chooses a Hartree-Fock (HF) state as the reference (but not in the general case).

In the following, we will consider the expansion with respect to the uncorrelated propagator $g^{(0)}(\omega)$ that is associated with a mean-field reference state $\left|\phi_{0}^{A}\right\rangle$. Hence, we redefine the transition amplitudes of the unperturbed $(A \pm 1)$-body systems, denoted by $\left|\phi^{(A \pm 1)}\right\rangle$, as follows:

$$
Z_{\alpha}^{i=n, k} \equiv\left\{\begin{array}{l}
\left(X_{\alpha}^{n}\right)^{*} \equiv\left\langle\phi_{0}^{A}\left|a_{\alpha}\right| \phi_{n}^{A+1}\right\rangle \\
Y_{\alpha}^{k} \equiv\left\langle\phi_{k}^{A-1}\left|a_{\alpha}\right| \phi_{0}^{A}\right\rangle
\end{array}\right.
$$

which build the reference propagator similarly to Eq. (4). In general, to obtain the $\mathrm{ADC}(3)$ approximation for such reference state, one only needs to substitute the amplitudes from Eq. (6) in the expressions for Eqs. (A1)-(A4) with the corresponding ones from Eq. (C1). Note that, differently from the true Dyson eigenstates of Eq. (6), the orbitals $n$ and $k$ of Eq. (C1) form a complete orthonormal set. Thus, the great advantage in using a mean-field reference state is that the numbers of particle and hole states is drastically reduced and, in fact, tractable. In the following we will consider the most general case in which these orbits are different from the model space basis $\{\alpha\}$, then the $Z_{\alpha}^{i}$ give the unitary transformation between the two sets. In standard applications of perturbation theory it is customary to identify the basis $\{\alpha\}$ with the un- perturbed orbits of the reference state. One can always reduce to this particular case by substituting $X_{\alpha}^{n} \rightarrow \delta_{n \alpha}$ and $Y_{\alpha}^{k} \rightarrow \delta_{k \alpha}$, which recovers the expressions reported in Refs. [20, 21].

\section{Static self-energy}

Equation 14 for the 1B effective interaction is exact and it is given in terms of correlated $1 \mathrm{~B}$ and $2 \mathrm{~B}$ propagators: for this reason, practical calculations (such as the above mentioned $s c 0$ approach) may follow an iterative procedure to "dress" the propagator and evaluate selfconsistently the first-order static irreducible self-energy. Alternatively, one can consider the explicit expansion of $\Sigma_{\alpha \beta}^{\infty}$ in terms of uncorrelated propagators. This should be done up to the same order $n$ that matches the $\operatorname{ADC}(n)$ truncation for $\widetilde{\Sigma}_{\alpha \beta}(\omega)$. Thus, we expand the effective interaction $\widetilde{U}$ as follows:

$$
\widetilde{U}=\sum_{\alpha \beta}\left(\widetilde{U}_{\alpha \beta}^{(1)}+\widetilde{U}_{\alpha \beta}^{(2)}+\widetilde{U}_{\alpha \beta}^{(3)}+\ldots\right) a_{\alpha}^{\dagger} a_{\beta} .
$$

In a similar fashion, one considers the expansion (up to second order) of $\widetilde{V}$, that is

$$
\widetilde{V}=\sum_{\alpha \beta, \gamma \delta}\left(\widetilde{V}_{\alpha \beta, \gamma \delta}^{(1)}+\widetilde{V}_{\alpha \beta, \gamma \delta}^{(2)}+\ldots\right) a_{\alpha}^{\dagger} a_{\beta}^{\dagger} a_{\delta} a_{\gamma} .
$$

The first-order term in Eq. $(\mathrm{C} 2)$ is given by the expression in Eq. 14 once correlated propagators are substituted with bare ones. It is composed by the three diagrams represented in Fig. 9 and can be written in terms of one-body reduced density matrix, $\rho_{\alpha \beta}^{(0)}=-i \hbar g_{\alpha \beta}^{(0)}\left(t-t^{+}\right)$, which is the uncorrelated version of Eq. 16). We have

$$
\widetilde{U}_{\alpha \beta}^{(1)}=-U_{\alpha \beta}+\sum_{\gamma \delta} V_{\alpha \gamma, \beta \delta} \rho_{\delta \gamma}^{(0)}+\frac{1}{4} \sum_{\substack{\gamma \delta \\ \epsilon \eta}} W_{\alpha \gamma \epsilon, \beta \delta \eta} \rho_{\delta \gamma}^{(0)} \rho_{\eta \epsilon}^{(0)} .
$$

Similarly, the explicit expression for the matrix element $\widetilde{V}_{\alpha \beta, \gamma \delta}^{(1)}$ is depicted in Fig. 10 . It can be directly read from Eq. 15 once the correlated fermionic loop is substituted with an uncorrelated one:

$$
\widetilde{V}_{\alpha \beta, \gamma \delta}^{(1)}=V_{\alpha \beta, \gamma \delta}+\sum_{\epsilon \eta} W_{\alpha \beta \epsilon, \gamma \delta \eta} \rho_{\eta, \epsilon}^{(0)} .
$$

The second-order term $\widetilde{U}^{(2)}$ is composed by eight different Feynman diagrams, that can be grouped into four by 


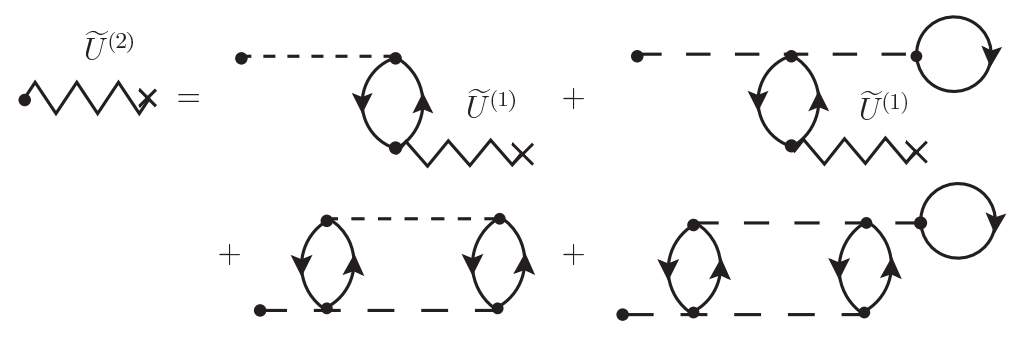

FIG. 11. As described in the caption of Fig. 9 but for the second-order term $\widetilde{U}^{(2)}$.

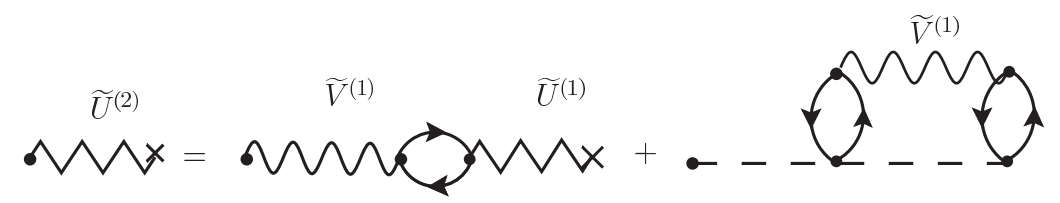

FIG. 12. As described in the caption of Fig. 9 but for the second-order term $\widetilde{U}^{(2)}$ rewritten in terms of $\widetilde{V}^{(1)}$.

using the effective interaction $\widetilde{U}^{(1)}$ :

$$
\begin{aligned}
\widetilde{U}_{\alpha \beta}^{(2)}= & -i \hbar \int \frac{d \omega}{2 \pi} \sum_{\substack{\epsilon \eta \\
\gamma \delta}}\left[V_{\alpha \gamma, \beta \delta}+\sum_{\epsilon \eta} W_{\alpha \gamma \epsilon, \beta \delta \eta} \rho_{\eta \epsilon}^{(0)}\right] g_{\delta \epsilon}^{(0)}(\omega) g_{\eta \gamma}^{(0)}(\omega) \widetilde{U}_{\epsilon \eta}^{(1)} \\
& +\frac{(i \hbar)^{3}}{4} \int \frac{\mathrm{d} \omega_{1}}{2 \pi} \int \frac{\mathrm{d} \omega_{2}}{2 \pi} \int \frac{\mathrm{d} \omega_{3}}{2 \pi} \sum_{\substack{\epsilon \eta \nu \delta \\
\gamma \lambda \mu \rho}} W_{\alpha \gamma \epsilon, \beta \delta \eta} g_{\nu \gamma}^{(0)}\left(\omega_{1}\right) g_{\delta \rho}^{(0)}\left(\omega_{2}\right) g_{\lambda \epsilon}^{(0)}\left(\omega_{3}\right) g_{\eta \mu}^{(0)}\left(\omega_{1}+\omega_{3}-\omega_{2}\right)\left[V_{\rho \mu, \nu \lambda}+\sum_{\epsilon \eta} W_{\rho \mu \epsilon, \nu \lambda \eta} \rho_{\eta \epsilon}^{(0)}\right]
\end{aligned}
$$

with the corresponding diagrams displayed in Fig. 11. The terms in Eq. (C6) can be further reduced by using the effective $2 \mathrm{NF}$ at the first order,

$$
\begin{aligned}
\widetilde{U}_{\alpha \beta}^{(2)}= & -i \hbar \int \frac{d \omega}{2 \pi} \sum_{\substack{\epsilon \eta \\
\gamma \delta}} \widetilde{V}_{\alpha \gamma, \beta \delta}^{(1)} g_{\delta \epsilon}^{(0)}(\omega) g_{\eta \gamma}^{(0)}(\omega) \widetilde{U}_{\epsilon \eta}^{(1)} \\
& +\frac{(i \hbar)^{3}}{4} \int \frac{\mathrm{d} \omega_{1}}{2 \pi} \int \frac{\mathrm{d} \omega_{2}}{2 \pi} \int \frac{\mathrm{d} \omega_{3}}{2 \pi} \sum_{\substack{\epsilon \eta \nu \delta \\
\gamma \lambda \mu \rho}} W_{\alpha \gamma \epsilon, \beta \delta \eta} g_{\nu \gamma}^{(0)}\left(\omega_{1}\right) g_{\delta \rho}^{(0)}\left(\omega_{2}\right) g_{\lambda \epsilon}^{(0)}\left(\omega_{3}\right) g_{\eta \mu}^{(0)}\left(\omega_{1}+\omega_{3}-\omega_{2}\right) \widetilde{V}_{\rho \mu, \nu \lambda}^{(1)},
\end{aligned}
$$

as depicted in Fig. 12 .

When the integration over the frequencies is performed, the second order term $\widetilde{U}^{(2)}$ becomes

$$
\begin{aligned}
\widetilde{U}_{\alpha \beta}^{(2)} & =\sum_{\substack{\epsilon \eta \\
\gamma \delta}} \widetilde{V}_{\alpha \gamma, \beta \delta}^{(1)} \widetilde{U}_{\epsilon \eta}^{(1)}\left(\sum_{n_{1} k_{2}} \frac{\left(X_{\delta}^{n_{1}}\right)^{*} X_{\epsilon}^{n_{1}} Y_{\eta}^{k_{2}}\left(Y_{\gamma}^{k_{2}}\right)^{*}}{-\left(\varepsilon_{n_{1}}^{+}-\varepsilon_{k_{2}}^{-}\right)+i \eta}-\sum_{k_{1} n_{2}} \frac{Y_{\delta}^{k_{1}}\left(Y_{\epsilon}^{k_{1}}\right)^{*}\left(X_{\eta}^{n_{2}}\right)^{*} X_{\gamma}^{n_{2}}}{-\left(\varepsilon_{k_{1}}^{-}-\varepsilon_{n_{2}}^{+}\right)-i \eta}\right) \\
& +\frac{1}{4} \sum_{\substack{\epsilon \eta \nu \delta \\
\gamma \lambda \mu \rho}} W_{\alpha \gamma \epsilon, \beta \delta \eta}\left(\sum_{\substack{n_{1} n_{2} \\
k_{3} k_{4}}} \frac{\left(X_{\nu}^{n_{1}} X_{\lambda}^{n_{2}} Y_{\rho}^{k_{3}} Y_{\mu}^{k_{4}}\right)^{*} X_{\gamma}^{n_{1}} X_{\epsilon}^{n_{2}} Y_{\delta}^{k_{3}} Y_{\eta}^{k_{4}}}{-\left(\varepsilon_{n_{1}}^{+}+\varepsilon_{n_{2}}^{+}-\varepsilon_{k_{3}}^{-}-\varepsilon_{k_{4}}^{-}\right)+i \eta}-\sum_{\substack{k_{1} k_{2} \\
n_{3} n_{4}}} \frac{Y_{\nu}^{k_{1}} Y_{\lambda}^{k_{2}} X_{\rho}^{n_{3}} X_{\mu}^{n_{4}}\left(Y_{\gamma}^{k_{1}} Y_{\epsilon}^{k_{2}} X_{\delta}^{n_{3}} X_{\eta}^{n_{4}}\right)^{*}}{-\left(\varepsilon_{k_{1}}^{-}+\varepsilon_{k_{2}}^{-}-\varepsilon_{n_{3}}^{+}-\varepsilon_{n_{4}}^{+}\right)-i \eta}\right) \widetilde{V}_{\rho \mu, \nu \lambda}^{(1)} .
\end{aligned}
$$

Note that the last term on the right hand side of Eq. C8 corresponds the last diagram in Fig. 12 and it is of skeleton type. This is the second-order contribution to $\Sigma_{\alpha \beta}^{\infty}$ that would appear also in the self-consistent expansion.

As we discussed above, it is customary in several practical applications to assume for the model space the very same orbits that diagonalize the unperturbed Hamiltonian, $\hat{H}_{0}$, and define the reference state. In this case the amplitudes (C1) become diagonal in the two indexes $i$ and $\alpha$. Similarly, one may chose an HF reference state and in this case the term $\widetilde{U}^{(1)}$ vanishes because of the specific definition of the HF potential (as it is obvious from Eq. (C4)). Whether or not it is convenient to take these assumptions-and in particular which is the best reference state to 


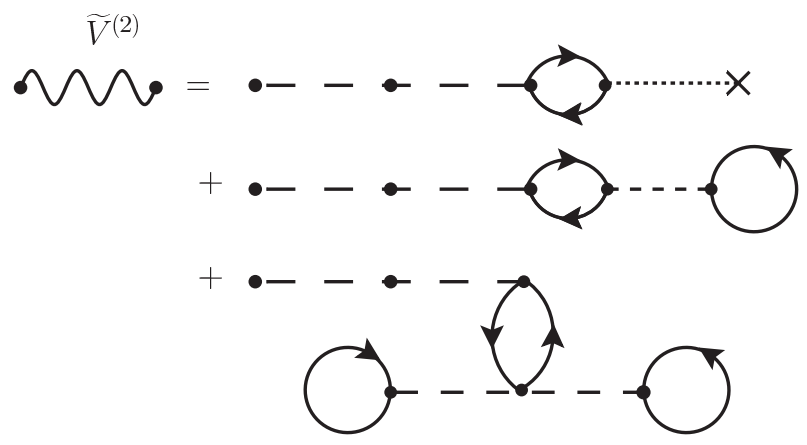

FIG. 13. As described in the caption of Fig. 10 but for the second-order term $\widetilde{V}^{(2)}$.

use-depends of the specific computational approach and on the specific system one needs to solve. For completeness, we give the example of how Eq. C8 would simplify if both these assumptions are made:

$$
\widetilde{U}_{\alpha \beta}^{(2)}=\left(\frac{1}{4} \sum_{\mu, \nu \notin F} \sum_{\rho, \lambda \in F} V_{\rho \lambda, \mu \nu} \frac{1}{\left(\varepsilon_{\rho}^{-}+\varepsilon_{\lambda}^{-}-\varepsilon_{\mu}^{+}-\varepsilon_{\nu}^{+}\right)+i \eta} W_{\alpha \mu \nu, \beta \rho \lambda}+W_{\alpha \rho \lambda, \beta \mu \nu} \frac{1}{\left(\varepsilon_{\rho}^{-}+\varepsilon_{\lambda}^{-}-\varepsilon_{\mu}^{+}-\varepsilon_{\nu}^{+}\right)+i \eta} V_{\mu \nu, \rho \lambda}\right),
$$

where we have used the notations $\in F(\notin F)$ to restrict the sums over occupied (unoccupied) orbits.

For the second-order term $\widetilde{V}_{\alpha \beta, \gamma \delta}^{(2)}$ in the expansion of Eq. $\mathrm{C} 3$, we have the three diagrams on the right-hand side of Fig. 13 with the following expressions:

$$
\begin{aligned}
\widetilde{V}_{\alpha \beta, \gamma \delta}^{(2)}= & i \hbar \int \frac{\mathrm{d} \omega}{2 \pi} \sum_{\substack{\epsilon \eta \\
\lambda \mu}} W_{\alpha \beta \epsilon, \gamma \delta \eta} g_{\mu \epsilon}^{(0)}(\omega) g_{\eta \nu}^{(0)}(\omega) U_{\nu \mu}-i \hbar \int \frac{\mathrm{d} \omega}{2 \pi} \sum_{\substack{\epsilon \nu \\
\lambda \mu \rho}} W_{\alpha \beta \epsilon, \gamma \delta \eta} g_{\mu \epsilon}^{(0)}(\omega) g_{\eta \nu}^{(0)}(\omega) \rho_{\lambda \rho}^{(0)} V_{\nu \rho, \mu \lambda} \\
& -\frac{i \hbar}{2} \int \frac{\mathrm{d} \omega}{2 \pi} \sum_{\substack{\epsilon \eta \nu \sigma \\
\lambda \mu \rho \tau}} W_{\alpha \beta \epsilon, \gamma \delta \eta} g_{\mu \epsilon}^{(0)}(\omega) g_{\eta \nu}^{(0)}(\omega) W_{\nu \lambda \sigma, \mu \rho \tau} \rho_{\rho \lambda}^{(0)} \rho_{\tau \sigma}^{(0)} \\
= & -i \hbar \int \frac{\mathrm{d} \omega}{2 \pi} \sum_{\substack{\epsilon \eta \\
\nu \mu}} W_{\alpha \beta \epsilon, \gamma \delta \eta} g_{\mu \epsilon}^{(0)}(\omega) g_{\eta \nu}^{(0)}(\omega) \widetilde{U}_{\nu \mu}^{(1)}
\end{aligned}
$$

where in the last equality we have written a more compact expression for $\widetilde{V}^{(2)}$ by using the first term in the expansion of $\widetilde{U}$. The integration over the frequency in Eq. C10 gives the expression of $\widetilde{V}^{(2)}$ in terms of the uncorrelated transition amplitudes:

$$
\widetilde{V}_{\alpha \beta, \gamma \delta}^{(2)}=\sum_{\substack{\epsilon \eta \\ \mu \nu}} W_{\alpha \beta \epsilon, \gamma \delta \eta}\left(\sum_{n_{1} k_{2}} \frac{\left(X_{\mu}^{n_{1}} Y_{\nu}^{k_{2}}\right)^{*} X_{\epsilon}^{n_{1}} Y_{\eta}^{k_{2}}}{-\left(\varepsilon_{n_{1}}^{+}-\varepsilon_{k_{2}}^{-}\right)+i \eta}-\sum_{k_{1} n_{2}} \frac{Y_{\mu}^{k_{1}} X_{\nu}^{n_{2}}\left(Y_{\epsilon}^{k_{1}} X_{\eta}^{n_{2}}\right)^{*}}{-\left(\varepsilon_{k_{1}}^{-}-\varepsilon_{n_{2}}^{+}\right)-i \eta}\right) \widetilde{U}_{\nu \mu}^{(1)},
$$

which is zero in the specific case of an HF reference state, due to $\widetilde{U}^{(1)}$ vanishing.

The expansion of $\widetilde{U}$ in Eq. $\left[\right.$ C2 2 contains also the term $\widetilde{U}_{\alpha \beta}^{(3)}$ composed by the 14 contributions shown in Fig. 14. By using the same Feynman rules applied for the terms at second and third order (see Appendix A of Ref. [22]), one can derive the expressions for those fourteen diagrams. Here we give the final equations after all integrals over the frequencies have been performed. Using the compact notation for npnh coupled cluster amplitudes of Eqs. (46)- (48) and assuming Einstein's summing convention throughout, they are listed below according to the order of appearance in Fig. 14 .

$$
\begin{aligned}
\widetilde{U}_{\alpha \beta}^{(3)} \text { (14a) }= & \widetilde{V}_{\alpha \gamma, \beta \delta}^{(1)}\left(X_{\delta}^{n_{1}}\right)^{*} X_{\gamma}^{n_{2}} t_{k_{3}}^{n_{1}}\left(t_{k_{3}}^{n_{2}}\right)^{*}-\widetilde{V}_{\alpha \gamma, \beta \delta}^{(1)}\left(Y_{\gamma}^{k_{2}}\right)^{*} Y_{\delta}^{k_{1}} t_{k_{2}}^{n_{3}}\left(t_{k_{1}}^{n_{3}}\right)^{*} \\
& +\widetilde{V}_{\alpha \gamma, \beta \delta}^{(1)} \widetilde{U}_{\epsilon \eta}^{(1)} \widetilde{U}_{\mu \nu}^{(1)}\left(\frac{\left(X_{\nu}^{n_{1}} X_{\eta}^{n_{2}} Y_{\epsilon}^{k_{3}}\right)^{*} X_{\gamma}^{n_{1}} X_{\mu}^{n_{2}} Y_{\delta}^{k_{3}}}{\left(-\left(\varepsilon_{n_{1}}^{+}-\varepsilon_{k_{3}}^{-}\right)+i \eta\right)\left(-\left(\varepsilon_{n_{2}}^{+}-\varepsilon_{k_{3}}^{-}\right)+i \eta\right)}-\frac{\left(Y_{\gamma}^{k_{1}} Y_{\mu}^{k_{2}} X_{\delta}^{n_{3}}\right)^{*} Y_{\nu}^{k_{1}} Y_{\eta}^{k_{2}} X_{\epsilon}^{n_{3}}}{\left(-\left(\varepsilon_{k_{2}}^{-}-\varepsilon_{n_{3}}^{+}\right)-i \eta\right)\left(-\left(\varepsilon_{k_{1}}^{-}-\varepsilon_{n_{3}}^{+}\right)-i \eta\right)}\right. \\
& \left.+\frac{\left(X_{\delta}^{n_{1}} X_{\eta}^{n_{2}} Y_{\gamma}^{k_{3}}\right)^{*} X_{\epsilon}^{n_{1}} X_{\mu}^{n_{2}} Y_{\nu}^{k_{3}}}{\left(-\left(\varepsilon_{n_{2}}^{+}-\varepsilon_{k_{3}}^{-}\right)+i \eta\right)\left(-\left(\varepsilon_{n_{1}}^{+}-\varepsilon_{k_{3}}^{-}\right)+i \eta\right)}-\frac{\left(Y_{\mu}^{k_{1}} Y_{\epsilon}^{k_{2}} X_{\nu}^{n_{3}}\right)^{*} Y_{\eta}^{k_{1}} Y_{\delta}^{k_{2}} X_{\gamma}^{n_{3}}}{\left(-\left(\varepsilon_{k_{1}}^{-}-\varepsilon_{n_{3}}^{+}\right)-i \eta\right)\left(-\left(\varepsilon_{k_{2}}^{-}-\varepsilon_{n_{3}}^{+}\right)-i \eta\right)}\right)
\end{aligned}
$$




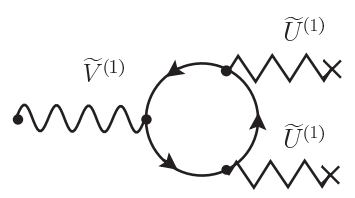

(a)

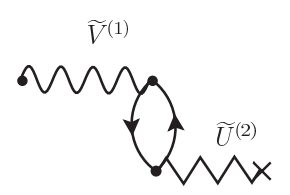

(b)

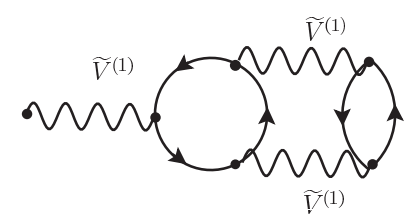

(c)

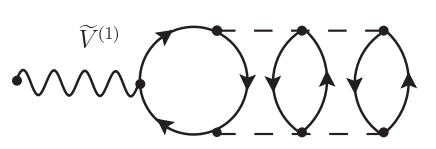

(d)

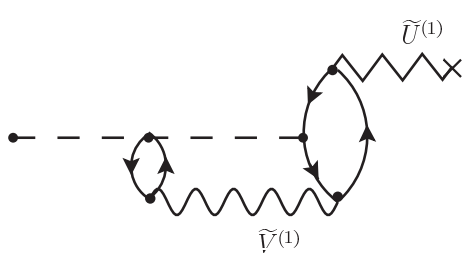

(g)

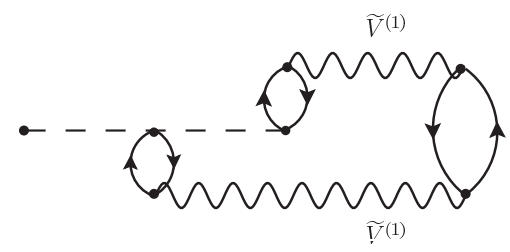

(j)

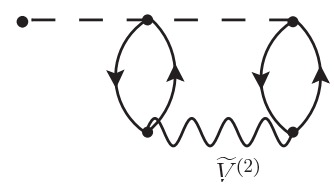

(e)

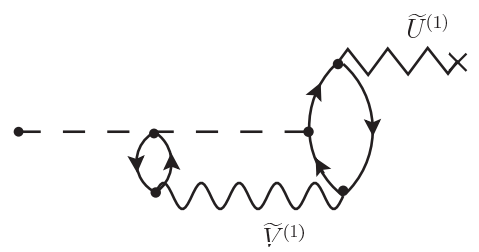

(h)

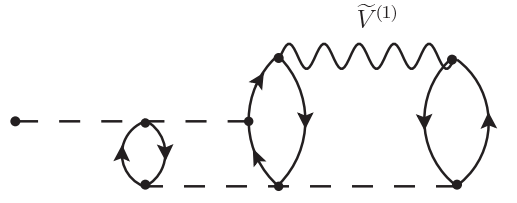

(k)

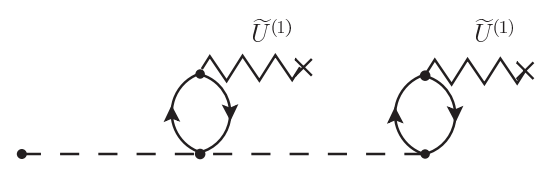

(f)

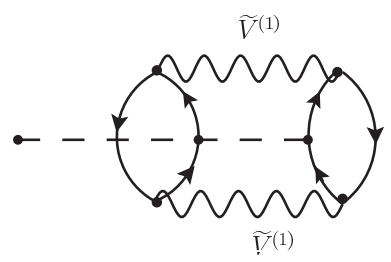

(i)

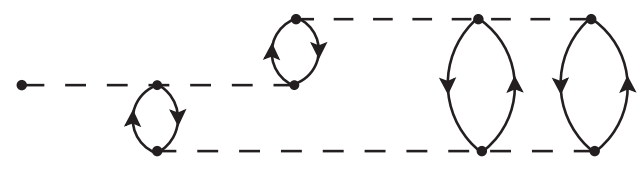

(m)

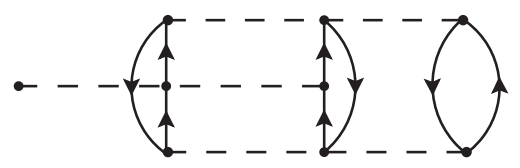

(n)

FIG. 14. As described in the caption of Fig. 9 but for the third-order term $\widetilde{U}^{(3)}$.

$$
\widetilde{U}_{\alpha \beta}^{(3)}(14 \mathrm{~b})=\widetilde{V}_{\alpha \gamma, \beta \delta}^{(1)}\left(\left(X_{\delta}^{n_{1}} Y_{\gamma}^{k_{2}}\right)^{*} t_{k_{2}}^{n_{1}}-\left(t_{k_{1}}^{n_{2}}\right)^{*} X_{\gamma}^{n_{2}} Y_{\delta}^{k_{1}}\right)
$$

$$
\begin{aligned}
\widetilde{U}_{\alpha \beta}^{(3)}(\underline{14 \mathrm{c})}= & -\frac{1}{2} \widetilde{V}_{\alpha \gamma, \beta \delta}^{(1)}\left(\left(X_{\delta}^{n_{2}}\right)^{*} X_{\gamma}^{n_{1}}\left(t_{k_{4} k_{5}}^{n_{1} n_{3}}\right)^{*} t_{k_{4} k_{5}}^{n_{2} n_{3}}+\left(Y_{\gamma}^{k_{2}}\right)^{*} Y_{\delta}^{k_{1}}\left(t_{k_{1} k_{3}}^{n_{4} n_{5}}\right)^{*} t_{k_{2} k_{3}}^{n_{4} n_{5}}\right) \\
& -\frac{1}{2} \widetilde{V}_{\alpha \gamma, \beta \delta}^{(1)} V_{\mu \tau, \nu \zeta}^{(1)}\left(\frac{\left(X_{\nu}^{n_{2}}\right)^{*} X_{\mu}^{n_{1}} X_{\gamma}^{n_{2}} X_{\tau}^{n_{3}} Y_{\zeta}^{k_{4}} Y_{\delta}^{k_{5}}}{\left(-\left(\varepsilon_{k_{5}}^{-}-\varepsilon_{n_{2}}^{+}\right)-i \eta\right)}\left(t_{k_{4} k_{5}}^{n_{1} n_{3}}\right)^{*}+\frac{\left(Y_{\gamma}^{k_{1}} Y_{\mu}^{k_{2}} Y_{\tau}^{k_{3}} X_{\delta}^{n_{4}} X_{\zeta}^{n_{5}}\right)^{*} Y_{\nu}^{k_{1}}}{\left(-\left(\varepsilon_{k_{1}}^{-}-\varepsilon_{n_{4}}^{+}\right)-i \eta\right)} t_{k_{2} k_{3}}^{n_{4} n_{5}}\right) \\
& -\frac{1}{2} \widetilde{V}_{\alpha \gamma, \beta \delta}^{(1)} \widetilde{V}_{\epsilon \sigma, \eta \rho}^{(1)}\left(\frac{\left(X_{\delta}^{n_{1}} X_{\eta}^{n_{2}} X_{\rho}^{n_{3}} Y_{\gamma}^{k_{4}} Y_{\sigma}^{k_{5}}\right)^{*} X_{\epsilon}^{n_{1}}}{\left(-\left(\varepsilon_{k_{4}}^{-}-\varepsilon_{n_{1}}^{+}\right)-i \eta\right)} t_{k_{4} k_{5}}^{n_{2} n_{3}}+\frac{\left(Y_{\epsilon}^{k_{1}}\right)^{*} Y_{\delta}^{k_{1}} Y_{\eta}^{k_{2}} Y_{\rho}^{k_{3}} X_{\gamma}^{n_{4}} X_{\sigma}^{n_{5}}}{\left(-\left(\varepsilon_{k_{1}}^{-}-\varepsilon_{n_{4}}^{+}\right)-i \eta\right)}\left(t_{k_{2} k_{3}}^{n_{4} n_{5}}\right)^{*}\right) ;(\mathrm{C} 1)
\end{aligned}
$$




$$
\begin{aligned}
& \widetilde{U}_{\alpha \beta}^{(3)} \underline{(14 \mathrm{~d})}=\frac{1}{12} \widetilde{V}_{\alpha \gamma, \beta \delta}^{(1)}\left(-\left(X_{\delta}^{n_{2}}\right)^{*} X_{\gamma}^{n_{1}}\left(t_{k_{5} k_{6} k_{7}}^{n_{1} n_{3} n_{4}}\right)^{*} t_{k_{5} k_{6} k_{7}}^{n_{2} n_{3} n_{4}}-\left(Y_{\gamma}^{k_{1}}\right)^{*} Y_{\delta}^{k_{2}}\left(t_{k_{2} k_{3} k_{4}}^{n_{5} n_{6} n_{7}}\right)^{*} t_{k_{1} k_{3} k_{4}}^{n_{5} n_{6} n_{7}}\right) \\
& +\frac{1}{12} \widetilde{V}_{\alpha \gamma, \beta \delta}^{(1)} W_{\chi \eta \epsilon, \lambda \nu \mu}\left(\frac{\left(X_{\lambda}^{n_{1}}\right)^{*} X_{\gamma}^{n_{1}} X_{\chi}^{n_{2}} X_{\eta}^{n_{3}} X_{\epsilon}^{n_{4}} Y_{\delta}^{k_{5}} Y_{\nu}^{k_{6}} Y_{\mu}^{k_{7}}}{\left(-\left(\varepsilon_{n_{1}}^{+}-\varepsilon_{k_{5}}^{-}\right)+i \eta\right)}\left(t_{k_{5} k_{6} k_{7}}^{n_{2} n_{3} n_{4}}\right)^{*}\right. \\
& \left.+\frac{\left(Y_{\gamma}^{k_{1}} Y_{\chi}^{k_{2}} Y_{\eta}^{k_{3}} Y_{\epsilon}^{k_{4}} X_{\delta}^{n_{5}} X_{\nu}^{n_{6}} X_{\mu}^{n_{7}}\right)^{*} Y_{\lambda}^{k_{1}}}{\left(-\left(\varepsilon_{n_{5}}^{+}-\varepsilon_{k_{1}}^{-}\right)+i \eta\right)} t_{k_{2} k_{3} k_{4}}^{n_{5} n_{6} n_{7}}\right) \\
& +\frac{1}{12} \widetilde{V}_{\alpha \gamma, \beta \delta}^{(1)} W_{\sigma \zeta \theta, \tau \rho \kappa}\left(\frac{\left(X_{\delta}^{n_{1}} X_{\tau}^{n_{2}} X_{\rho}^{n_{3}} X_{\kappa}^{n_{4}} Y_{\gamma}^{k_{5}} Y_{\zeta}^{k_{6}} Y_{\theta}^{k_{7}}\right)^{*} X_{\sigma}^{n_{1}}}{\left(-\left(\varepsilon_{n_{1}}^{+}-\varepsilon_{k_{5}}^{-}\right)+i \eta\right)} t_{k_{5} k_{6} k_{7}}^{n_{2} n_{3} n_{4}}\right. \\
& \left.+\frac{\left(Y_{\sigma}^{k_{1}}\right)^{*} Y_{\delta}^{k_{1}} Y_{\tau}^{k_{2}} Y_{\rho}^{k_{3}} Y_{\kappa}^{k_{4}} X_{\gamma}^{n_{5}} X_{\zeta}^{n_{6}} X_{\theta}^{n_{7}}}{\left(-\left(\varepsilon_{n_{5}}^{+}-\varepsilon_{k_{1}}^{-}\right)+i \eta\right)}\left(t_{k_{2} k_{3} k_{4}}^{n_{5} n_{6} n_{7}}\right)^{*}\right)
\end{aligned}
$$

$$
\begin{aligned}
\widetilde{U}_{\alpha \beta}^{(3)}(14 \mathrm{le})= & \frac{1}{4} \sum_{\substack{\epsilon \eta \nu \delta \\
\gamma \lambda \mu \rho}} W_{\alpha \gamma \epsilon, \beta \delta \eta}\left(\sum_{\substack{n_{1} n_{2} \\
k_{3} k_{4}}} \frac{\left(X_{\nu}^{n_{1}} X_{\lambda}^{n_{2}} Y_{\rho}^{k_{3}} Y_{\mu}^{k_{4}}\right)^{*} X_{\gamma}^{n_{1}} X_{\epsilon}^{n_{2}} Y_{\delta}^{k_{3}} Y_{\eta}^{k_{4}}}{-\left(\varepsilon_{n_{1}}^{+}+\varepsilon_{n_{2}}^{+}-\varepsilon_{k_{3}}^{-}-\varepsilon_{k_{4}}^{-}\right)+i \eta}\right. \\
& \left.-\sum_{\substack{k_{1} k_{2} \\
n_{3} n_{4}}} \frac{Y_{\nu}^{k_{1}} Y_{\lambda}^{k_{2}} X_{\rho}^{n_{3}} X_{\mu}^{n_{4}}\left(Y_{\gamma}^{k_{1}} Y_{\epsilon}^{k_{2}} X_{\delta}^{n_{3}} X_{\eta}^{n_{4}}\right)^{*}}{-\left(\varepsilon_{k_{1}}^{-}+\varepsilon_{k_{2}}^{-}-\varepsilon_{n_{3}}^{+}-\varepsilon_{n_{4}}^{+}\right)-i \eta}\right) \widetilde{V}_{\rho \mu, \nu \lambda}^{(2)}
\end{aligned}
$$

$$
\begin{aligned}
\widetilde{U}_{\alpha \beta}^{(3)}(14 \mathrm{ff})= & W_{\alpha \gamma \epsilon, \beta \delta \eta}\left(\left(X_{\delta}^{n_{1}} X_{\eta}^{n_{2}} Y_{\gamma}^{k_{3}} Y_{\epsilon}^{k_{4}}\right)^{*} t_{k_{3}}^{n_{1}} t_{k_{4}}^{n_{2}}+\left(X_{\delta}^{n_{1}} Y_{\gamma}^{k_{3}}\right)^{*} X_{\epsilon}^{n_{2}} Y_{\eta}^{k_{4}} t_{k_{3}}^{n_{1}}\left(t_{k_{4}}^{n_{2}}\right)^{*}\right. \\
& \left.+\left(Y_{\epsilon}^{k_{2}} X_{\eta}^{n_{4}}\right)^{*} Y_{\delta}^{k_{1}} X_{\gamma}^{n_{3}} t_{k_{2}}^{n_{4}}\left(t_{k_{1}}^{n_{3}}\right)^{*}+Y_{\delta}^{k_{1}} Y_{\eta}^{k_{2}} X_{\gamma}^{n_{3}} X_{\epsilon}^{n_{4}}\left(t_{k_{2}}^{n_{4}}\right)^{*}\left(t_{k_{1}}^{n_{3}}\right)^{*}\right)
\end{aligned}
$$

$$
\begin{aligned}
\widetilde{U}_{\alpha \beta}^{(3)}(14 \mathrm{lg})= & -\frac{1}{2} W_{\alpha \gamma \epsilon, \beta \delta \eta}\left(-\left(X_{\eta}^{n_{1}}\right)^{*} X_{\epsilon}^{n_{2}} X_{\gamma}^{n_{3}} Y_{\delta}^{k_{5}} t_{k_{4}}^{n_{1}}\left(t_{k_{5} k_{4}}^{n_{3} n_{2}}\right)^{*}+\left(Y_{\epsilon}^{k_{2}} Y_{\gamma}^{k_{3}} X_{\delta}^{n_{5}}\right)^{*} Y_{\eta}^{k_{1}}\left(t_{k_{1}}^{n_{4}}\right)^{*} t_{k_{3} k_{2}}^{n_{5} n_{4}}\right) \\
& -\frac{1}{2} W_{\alpha \gamma \epsilon, \beta \delta \eta} U_{\sigma \chi}^{(1)}\left(-\frac{\left(X_{\eta}^{n_{1}} X_{\chi}^{n_{2}} X_{\delta}^{n_{3}} Y_{\epsilon}^{k_{4}} Y_{\gamma}^{k_{5}}\right)^{*} X_{\sigma}^{n_{1}}}{\left(-\left(\varepsilon_{n_{1}}^{+}+\varepsilon_{n_{3}}^{+}-\varepsilon_{k_{4}}^{-}-\varepsilon_{k_{5}}^{-}\right)+i \eta\right)} t_{k_{5} k_{4}}^{n_{3} n_{2}}+\frac{\left(Y_{\sigma}^{k_{1}}\right)^{*} Y_{\eta}^{k_{1}} Y_{\chi}^{k_{2}} Y_{\delta}^{k_{3}} X_{\gamma}^{n_{4}} X_{\epsilon}^{n_{5}}}{\varepsilon_{k_{1}}^{-}+\varepsilon_{k_{3}}^{-}-\varepsilon_{n_{4}}^{+}-\varepsilon_{n_{5}}^{+}+i \eta}\left(t_{k_{3} k_{2}}^{n_{4} n_{5}}\right)^{*}\right) \\
& -\frac{1}{2} W_{\alpha \gamma \epsilon, \beta \delta \eta} \widetilde{V}_{\mu \lambda, \nu \rho}^{(1)}\left(-\frac{\left(X_{\rho}^{n_{1}} X_{\nu}^{n_{3}} Y_{\mu}^{k_{5}}\right)^{*} X_{\epsilon}^{n_{1}} X_{\lambda}^{n_{2}} X_{\gamma}^{n_{3}} Y_{\eta}^{k_{4}} Y_{\delta}^{k_{5}}}{\left(-\left(\varepsilon_{n_{1}}^{+}+\varepsilon_{n_{3}}^{+}-\varepsilon_{k_{4}}^{-}-\varepsilon_{k_{5}}^{-}\right)+i \eta\right)}\left(t_{k_{4}}^{n_{2}}\right)^{*}\right. \\
& +\frac{\left.\left(Y_{\epsilon}^{k_{1}} Y_{\lambda}^{k_{2}} Y_{\gamma}^{k_{3}} X_{\eta}^{n_{4}} X_{\delta}^{n_{5}}\right)^{*} Y_{\rho}^{k_{1}} Y_{\nu}^{k_{3}} X_{\mu}^{n_{5}} t_{k_{2}}^{n_{4}}\right)}{\varepsilon_{k_{1}}^{-}+\varepsilon_{k_{3}}^{-}-\varepsilon_{n_{4}}^{+}-\varepsilon_{n_{5}}^{+}+i \eta}
\end{aligned}
$$

$$
\begin{aligned}
\widetilde{U}_{\alpha \beta}^{(3)}(14 \mathrm{~h})= & -\frac{1}{2} W_{\alpha \gamma \epsilon, \beta \delta \eta}\left(-\left(X_{\eta}^{n_{1}} X_{\delta}^{n_{3}} Y_{\gamma}^{k_{5}}\right)^{*} X_{\epsilon}^{n_{2}}\left(t_{k_{4}}^{n_{2}}\right)^{*} t_{k_{5} k_{4}}^{n_{3} n_{1}}+\left(Y_{\epsilon}^{k_{2}}\right)^{*} Y_{\eta}^{k_{1}} Y_{\delta}^{k_{3}} X_{\gamma}^{n_{5}} t_{k_{2}}^{n_{4}}\left(t_{k_{3} k_{1}}^{n_{5} n_{4}}\right)^{*}\right) \\
& -\frac{1}{2} W_{\alpha \gamma \epsilon, \beta \delta \eta} \widetilde{U}_{\sigma \chi}^{(1)}\left(-\frac{\left(X_{\chi}^{n_{2}}\right)^{*} X_{\sigma}^{n_{1}} X_{\epsilon}^{n_{2}} X_{\gamma}^{n_{3}} Y_{\eta}^{k_{4}} Y_{\delta}^{k_{5}}}{\left(-\left(\varepsilon_{n_{2}}^{+}+\varepsilon_{n_{3}}^{+}-\varepsilon_{k_{4}}^{-}-\varepsilon_{k_{5}}^{-}\right)+i \eta\right)}\left(t_{k_{5} k_{4}}^{n_{3} n_{1}}\right)^{*}\right. \\
& \left.+\frac{\left(Y_{\epsilon}^{k_{1}} Y_{\sigma}^{k_{2}} Y_{\gamma}^{k_{3}} X_{\eta}^{n_{4}} X_{\delta}^{n_{5}}\right)^{*} Y_{\chi}^{k_{1}}}{\varepsilon_{k_{1}}^{-}+\varepsilon_{k_{3}}^{-}-\varepsilon_{n_{4}}^{+}-\varepsilon_{n_{5}}^{+}+i \eta} t_{k_{3} k_{2}}^{n_{5} n_{4}}\right) \\
& -\frac{1}{2} W_{\alpha \gamma \epsilon, \beta \delta \eta} \widetilde{V}_{\mu \lambda, \nu \rho}^{(1)}\left(-\frac{\left(X_{\eta}^{n_{1}} X_{\rho}^{n_{2}} X_{\delta}^{n_{3}} Y_{\epsilon}^{k_{4}} Y_{\gamma}^{k_{5}}\right)^{*} X_{\lambda}^{n_{1}} X_{\mu}^{n_{3}} Y_{\nu}^{k_{5}} t_{k_{4}}^{n_{2}}}{\left(-\left(\varepsilon_{n_{1}}^{+}+\varepsilon_{n_{3}}^{+}-\varepsilon_{k_{4}}^{-}-\varepsilon_{k_{5}}^{-}\right)+i \eta\right)}\right. \\
& \left.+\frac{\left(Y_{\lambda}^{k_{2}} Y_{\mu}^{k_{3}} X_{\nu}^{n_{5}}\right)^{*} Y_{\rho}^{k_{1}} Y_{\eta}^{k_{2}} Y_{\delta}^{k_{3}} X_{\epsilon}^{n_{4}} X_{\gamma}^{n_{5}}}{\varepsilon_{k_{2}}^{-}+\varepsilon_{k_{3}}^{-}-\varepsilon_{n_{4}}^{+}-\varepsilon_{n_{5}}^{+}+i \eta}\right)
\end{aligned}
$$




$$
\begin{aligned}
\widetilde{U}_{\alpha \beta}^{(3)}(14 \mathrm{i})= & \frac{1}{8} W_{\alpha \gamma \epsilon, \beta \delta \eta}\left(\left(X_{\delta}^{n_{2}} X_{\eta}^{n_{4}}\right)^{*} X_{\gamma}^{n_{1}} X_{\epsilon}^{n_{3}}\left(t_{k_{5} k_{6}}^{n_{1} n_{3}}\right)^{*} t_{k_{5} k_{6}}^{n_{2} n_{4}}+\left(Y_{\gamma}^{k_{1}} Y_{\epsilon}^{k_{3}}\right)^{*} Y_{\delta}^{k_{2}} Y_{\eta}^{k_{4}}\left(t_{k_{2} k_{4}}^{n_{5} n_{6}}\right)^{*} t_{k_{1} k_{3}}^{n_{5} n_{6}}\right) \\
& +\frac{1}{8} W_{\alpha \gamma \epsilon, \beta \delta \eta} \widetilde{V}_{\tau \sigma, \zeta \chi}^{(1)}\left(+\frac{\left(X_{\zeta}^{n_{1}} X_{\chi}^{n_{3}}\right)^{*} X_{\gamma}^{n_{1}} X_{\tau}^{n_{2}} X_{\epsilon}^{n_{3}} X_{\sigma}^{n_{4}} Y_{\delta}^{k_{5}} Y_{\eta}^{k_{6}}}{\left(-\left(\varepsilon_{n_{1}}^{+}+\varepsilon_{n_{3}}^{+}-\varepsilon_{k_{5}}^{-}-\varepsilon_{k_{6}}^{-}\right)+i \eta\right)}\left(t_{k_{5} k_{6}}^{n_{2} n_{4}}\right)^{*}\right. \\
& \left.+\frac{\left(Y_{\gamma}^{k_{1}} Y_{\tau}^{k_{2}} Y_{\epsilon}^{k_{3}} Y_{\sigma}^{k_{4}} X_{\delta}^{n_{5}} X_{\eta}^{n_{6}}\right)^{*} Y_{\zeta}^{k_{1}} Y_{\chi}^{k_{3}}}{\varepsilon_{k_{1}}^{-}+\varepsilon_{k_{3}}^{-}-\varepsilon_{n_{5}}^{+}-\varepsilon_{n_{6}}^{+}+i \eta} t_{k_{2} k_{4}}^{n_{5} n_{6}}\right) \\
& +\frac{1}{8} W_{\alpha \gamma \epsilon, \beta \delta \eta} \widetilde{V}_{\mu \lambda, \nu \rho}^{(1)}\left(+\frac{\left(X_{\nu}^{n_{1}} X_{\delta}^{n_{2}} X_{\rho}^{n_{3}} X_{\eta}^{n_{4}} Y_{\gamma}^{k_{5}} Y_{\epsilon}^{k_{6}}\right)^{*} X_{\mu}^{n_{2}} X_{\lambda}^{n_{4}}}{\left(-\left(\varepsilon_{n_{2}}^{+}+\varepsilon_{n_{4}}^{+}-\varepsilon_{k_{5}}^{-}-\varepsilon_{k_{6}}^{-}\right)+i \eta\right)} t_{k_{5} k_{6}}^{n_{1} n_{3}}\right. \\
& \left.+\frac{\left(Y_{\mu}^{k_{2}} Y_{\lambda}^{k_{4}}\right)^{*} Y_{\nu}^{k_{1}} Y_{\delta}^{k_{2}} Y_{\rho}^{k_{3}} Y_{\eta}^{k_{4}} X_{\gamma}^{n_{5}} X_{\epsilon}^{n_{6}}}{\varepsilon_{k_{2}}^{-}+\varepsilon_{k_{4}}^{-}-\varepsilon_{n_{5}}^{+}-\varepsilon_{n_{6}}^{+}+i \eta}\left(t_{k_{1} k_{3}}^{n_{5} n_{6}}\right)^{*}\right)
\end{aligned}
$$

$$
\begin{aligned}
\widetilde{U}_{\alpha \beta}^{(3)}[14 \mathrm{j}]= & \frac{1}{2} W_{\alpha \gamma \epsilon, \beta \delta \eta}\left(\left(X_{\delta}^{n_{1}} Y_{\gamma}^{k_{4}}\right)^{*} X_{\epsilon}^{n_{2}} Y_{\eta}^{k_{5}}\left(t_{k_{5} k_{6}}^{n_{2} n_{3}}\right)^{*} t_{k_{4} k_{6}}^{n_{1} n_{3}}+\left(Y_{\epsilon}^{k_{2}} X_{\eta}^{n_{5}}\right)^{*} Y_{\delta}^{k_{1}} X_{\gamma}^{n_{4}}\left(t_{k_{1} k_{3}}^{n_{4} n_{6}}\right)^{*} t_{k_{2} k_{3}}^{n_{5} n_{6}}\right) \\
& +\frac{1}{2} W_{\alpha \gamma \epsilon, \beta \delta \eta} \widetilde{V}_{\mu \lambda, \nu \rho}^{(1)}\left(\frac{\left(X_{\nu}^{n_{1}} Y_{\mu}^{k_{4}}\right)^{*} X_{\gamma}^{n_{1}} X_{\lambda}^{n_{2}} X_{\epsilon}^{n_{3}} Y_{\delta}^{k_{4}} Y_{\rho}^{k_{5}} Y_{\eta}^{k_{6}}}{\left(-\left(\varepsilon_{n_{1}}^{+}+\varepsilon_{n_{3}}^{+}-\varepsilon_{k_{4}}^{-}-\varepsilon_{k_{6}}^{-}\right)+i \eta\right)}\left(t_{k_{6} k_{5}}^{n_{3} n_{2}}\right)^{*}\right. \\
& \left.+\frac{\left(Y_{\gamma}^{k_{1}} Y_{\lambda}^{k_{2}} Y_{\epsilon}^{k_{3}} X_{\delta}^{n_{4}} X_{\rho}^{n_{5}} X_{\eta}^{n_{6}}\right)^{*} Y_{\nu}^{k_{1}} X_{\mu}^{n_{4}}}{\varepsilon_{k_{2}}^{-}+\varepsilon_{k_{3}}^{-}-\varepsilon_{n_{5}}^{+}-\varepsilon_{n_{6}}^{+}+i \eta} t_{k_{3} k_{2}}^{n_{6} n_{5}}\right) \\
& +\frac{1}{2} W_{\alpha \gamma \epsilon, \beta \delta \eta} \widetilde{V}_{\tau \sigma, \zeta \chi}^{(1)}\left(\frac{\left(X_{\delta}^{n_{1}} X_{\eta}^{n_{2}} X_{\chi}^{n_{3}} Y_{\gamma}^{k_{4}} Y_{\epsilon}^{k_{5}} Y_{\sigma}^{k_{6}}\right)^{*} X_{\tau}^{n_{2}} Y_{\zeta}^{k_{5}}}{\left(-\left(\varepsilon_{n_{1}}^{+}+\varepsilon_{n_{2}}^{+}-\varepsilon_{k_{4}}^{-}-\varepsilon_{k_{5}}^{-}\right)+i \eta\right)} t_{k_{4} k_{6}}^{n_{1} n_{3}}\right. \\
& \left.+\frac{\left(Y_{\tau}^{k_{2}} X_{\zeta}^{n_{5}}\right)^{*} Y_{\delta}^{k_{1}} Y_{\eta}^{k_{2}} Y_{\chi}^{k_{3}} X_{\gamma}^{n_{4}} X_{\epsilon}^{n_{5}} X_{\sigma}^{n_{6}}}{\varepsilon_{k_{1}}^{-}+\varepsilon_{k_{2}}^{-}-\varepsilon_{n_{4}}^{+}-\varepsilon_{n_{5}}^{+}+i \eta}\left(t_{k_{1} k_{3}}^{n_{4} n_{6}}\right)^{*}\right)
\end{aligned}
$$

$$
\begin{aligned}
\widetilde{U}_{\alpha \beta}^{(3)}(141)= & \frac{1}{4} W_{\alpha \gamma \epsilon, \beta \delta \eta}\left(\frac{X_{\gamma}^{n_{2}} X_{\epsilon}^{n_{5}} Y_{\delta}^{k_{1}} Y_{\eta}^{k_{7}}}{\varepsilon_{n_{2}}^{+}+\varepsilon_{n_{5}}^{+}-\varepsilon_{k_{1}}^{-}-\varepsilon_{k_{7}}^{-}+i \eta}\left(t_{k_{1} k_{3} k_{6}}^{n_{2} n_{4} n_{5}}\right)^{*}\left(Y_{\mu}^{k_{7}}\right)^{*} X_{\tau}^{n_{4}} \widetilde{V}_{\mu \tau, \lambda \sigma}^{(1)} Y_{\lambda}^{k_{6}} Y_{\sigma}^{k_{3}}\right. \\
& +\frac{\left(Y_{\gamma}^{k_{2}} Y_{\epsilon}^{k_{5}} X_{\delta}^{n_{1}} X_{\eta}^{n_{7}}\right)^{*}}{\varepsilon_{k_{2}}^{-}+\varepsilon_{k_{5}}^{-}-\varepsilon_{n_{1}}^{+}-\varepsilon_{n_{7}}^{+}+i \eta} X_{\nu}^{n_{1}}\left(Y_{\kappa}^{k_{6}} Y_{\pi}^{k_{3}}\right)^{*} W_{\nu \kappa \pi, \rho \theta \phi} Y_{\rho}^{k_{2}} Y_{\theta}^{k_{5}}\left(X_{\phi}^{n_{4}}\right)^{*} t_{k_{3} k_{6}}^{n_{4} n_{7}} \\
& -X_{\gamma}^{n_{2}} X_{\epsilon}^{n_{5}} Y_{\delta}^{k_{1}}\left(X_{\eta}^{n_{7}}\right)^{*}\left(t_{k_{1} k_{3} k_{6}}^{n_{2} n_{4} n_{5}}\right)^{*} t_{k_{3} k_{6}}^{n_{4} n_{7}}+\left(Y_{\gamma}^{k_{2}} X_{\delta}^{n_{1}} Y_{\epsilon}^{k_{5}}\right)^{*} Y_{\eta}^{k_{7}} t_{k_{2} k_{4} k_{5}}^{n_{1} n_{3} n_{6}}\left(t_{k_{4} k_{7}}^{\left.n_{3} n_{6}\right)^{*}}\right. \\
& -\frac{X_{\gamma}^{n_{2}} X_{\epsilon}^{n_{5}} Y_{\delta}^{k_{1}} Y_{\eta}^{k_{7}}}{\varepsilon_{n_{2}}^{+}+\varepsilon_{n_{5}}^{+}-\varepsilon_{k_{1}}^{-}-\varepsilon_{k_{7}}^{-}+i \eta}\left(Y_{\nu}^{k_{1}}\right)^{*} X_{\kappa}^{n_{6}} X_{\pi}^{n_{3}} W_{\nu \kappa \pi, \rho \theta \phi}\left(X_{\rho}^{n_{2}} X_{\theta}^{n_{5}}\right)^{*} Y_{\phi}^{k_{4}}\left(t_{k_{4} k_{7}}^{n_{3} n_{6}}\right)^{*} \\
& \left.-\frac{\left(Y_{\gamma}^{k_{2}} Y_{\epsilon}^{k_{5}} X_{\delta}^{n_{1}} X_{\eta}^{n_{7}}\right)^{*}}{\varepsilon_{k_{2}}^{-}+\varepsilon_{k_{5}}^{-}-\varepsilon_{n_{1}}^{+}-\varepsilon_{n_{7}}^{+}+i \eta} t_{k_{2} k_{4} k_{5}}^{n_{1} n_{3} n_{6}} X_{\mu}^{n_{7}}\left(Y_{\tau}^{k_{4}}\right)^{*} \widetilde{V}_{\mu \tau, \lambda \sigma}^{(1)}\left(X_{\lambda}^{n_{6}} X_{\sigma}^{n_{3}}\right)^{*}\right)
\end{aligned}
$$




$$
\begin{aligned}
& \widetilde{U}_{\alpha \beta}^{(3)}(\underline{14 \mathrm{~m}})=\frac{1}{4} W_{\alpha \gamma \epsilon, \beta \delta \eta}\left(\left(X_{\delta}^{n_{1}} Y_{\gamma}^{k_{5}}\right)^{*} X_{\epsilon}^{n_{2}} Y_{\eta}^{k_{6}}\left(t_{k_{6} k_{7} k_{8}}^{n_{2} n_{3} n_{4}}\right)^{*} t_{k_{5} k_{7} k_{8}}^{n_{1} n_{3} n_{4}}+\left(Y_{\epsilon}^{k_{2}} X_{\eta}^{n_{6}}\right)^{*} Y_{\delta}^{k_{1}} X_{\gamma}^{n_{5}}\left(t_{k_{1} k_{3} k_{4}}^{n_{5} n_{7} n_{8}}\right)^{*} t_{k_{2} k_{3} k_{4}}^{n_{6} n_{7} n_{8}}\right) \\
& +\frac{1}{4} W_{\alpha \gamma \epsilon, \beta \delta \eta} W_{\nu \kappa \pi, \rho \theta \phi}\left(\frac{\left(X_{\rho}^{n_{2}} Y_{\nu}^{k_{6}}\right)^{*} X_{\epsilon}^{n_{1}} X_{\gamma}^{n_{2}} X_{\kappa}^{n_{3}} X_{\pi}^{n_{4}} Y_{\eta}^{k_{5}} Y_{\delta}^{k_{6}} Y_{\theta}^{k_{7}} Y_{\phi}^{k_{8}}}{\left(-\left(\varepsilon_{n_{1}}^{+}+\varepsilon_{n_{2}}^{+}-\varepsilon_{k_{5}}^{-}-\varepsilon_{k_{6}}^{-}\right)+i \eta\right)}\left(t_{k_{5} k_{7} k_{8}}^{n_{1} n_{3} n_{4}}\right)^{*}\right. \\
& \left.+\frac{\left(Y_{\epsilon}^{k_{1}} Y_{\gamma}^{k_{2}} Y_{\kappa}^{k_{3}} Y_{\pi}^{k_{4}} X_{\eta}^{n_{5}} X_{\delta}^{n_{6}} X_{\theta}^{n_{7}} X_{\phi}^{n_{8}}\right)^{*} Y_{\rho}^{k_{2}} X_{\nu}^{n_{6}}}{\varepsilon_{k_{1}}^{-}+\varepsilon_{k_{2}}^{-}-\varepsilon_{n_{5}}^{+}-\varepsilon_{n_{6}}^{+}+i \eta} t_{k_{1} k_{3} k_{4}}^{n_{5} n_{7} n_{8}}\right) \\
& +\frac{1}{4} W_{\alpha \gamma \epsilon, \beta \delta \eta} W_{\mu \tau \chi, \lambda \sigma \zeta}\left(\frac{\left(X_{\delta}^{n_{1}} X_{\eta}^{n_{2}} X_{\sigma}^{n_{3}} X_{\zeta}^{n_{4}} Y_{\gamma}^{k_{5}} Y_{\epsilon}^{k_{6}} Y_{\tau}^{k_{7}} Y_{\chi}^{k_{8}}\right)^{*} X_{\mu}^{n_{2}} Y_{\lambda}^{k_{6}}}{\left(-\left(\varepsilon_{n_{1}}^{+}+\varepsilon_{n_{2}}^{+}-\varepsilon_{k_{5}}^{-}-\varepsilon_{k_{6}}^{-}\right)+i \eta\right)} t_{k_{5} k_{7} k_{8}}^{n_{1} n_{3} n_{4}}\right. \\
& \left.+\frac{\left(Y_{\mu}^{k_{2}} X_{\lambda}^{n_{6}}\right)^{*} Y_{\delta}^{k_{1}} Y_{\eta}^{k_{2}} Y_{\sigma}^{k_{3}} Y_{\zeta}^{k_{4}} X_{\gamma}^{n_{5}} X_{\epsilon}^{n_{6}} X_{\tau}^{n_{7}} X_{\chi}^{n_{8}}}{\varepsilon_{k_{1}}^{-}+\varepsilon_{k_{2}}^{-}-\varepsilon_{n_{5}}^{+}-\varepsilon_{n_{6}}^{+}+i \eta}\left(t_{k_{1} k_{3} k_{4}}^{n_{5} n_{7} n_{8}}\right)^{*}\right) \\
& \widetilde{U}_{\alpha \beta}^{(3)}(\underline{14 n})=\frac{1}{24} W_{\alpha \gamma \epsilon, \beta \delta \eta}\left(\left(X_{\delta}^{n_{2}} X_{\eta}^{n_{4}}\right)^{*} X_{\gamma}^{n_{1}} X_{\epsilon}^{n_{3}}\left(t_{k_{6} k_{7} k_{8}}^{n_{1} n_{3} n_{5}}\right)^{*} t_{k_{6} k_{7} k_{8}}^{n_{2} n_{4} n_{5}}+\left(Y_{\gamma}^{k_{2}} Y_{\epsilon}^{k_{4}}\right)^{*} Y_{\delta}^{k_{1}} Y_{\eta}^{k_{3}}\left(t_{k_{1} k_{3} k_{5}}^{n_{6} n_{7} n_{8}}\right)^{*} t_{k_{2} k_{4} k_{5}}^{n_{6} n_{7} n_{8}}\right) \\
& +\frac{1}{24} W_{\alpha \gamma \epsilon, \beta \delta \eta} W_{\nu \kappa \pi, \rho \theta \phi}\left(\frac{\left(X_{\delta}^{n_{1}} X_{\rho}^{n_{2}} X_{\eta}^{n_{3}} X_{\theta}^{n_{4}} X_{\phi}^{n_{5}} Y_{\gamma}^{k_{6}} Y_{\epsilon}^{k_{7}} Y_{\pi}^{k_{8}}\right)^{*} X_{\nu}^{n_{1}} X_{\kappa}^{n_{3}}}{\left(-\left(\varepsilon_{n_{1}}^{+}+\varepsilon_{n_{3}}^{+}-\varepsilon_{k_{6}}^{-}-\varepsilon_{k_{7}}^{-}\right)+i \eta\right)} t_{k_{6} k_{7} k_{8} n_{4} n_{5}}^{n_{2}}\right. \\
& \left.+\frac{\left(Y_{\nu}^{k_{2}} Y_{\kappa}^{k_{4}}\right)^{*} Y_{\rho}^{k_{1}} Y_{\delta}^{k_{2}} Y_{\theta}^{k_{3}} Y_{\eta}^{k_{4}} Y_{\phi}^{k_{5}} X_{\gamma}^{n_{6}} X_{\epsilon}^{n_{7}} X_{\pi}^{n_{8}}}{\varepsilon_{k_{2}}^{-}+\varepsilon_{k_{4}}^{-}-\varepsilon_{n_{6}}^{+}-\varepsilon_{n_{7}}^{+}+i \eta}\left(t_{k_{1} k_{3} k_{5}}^{n_{6} n_{7} n_{8}}\right)^{*}\right) \\
& +\frac{1}{24} W_{\alpha \gamma \epsilon, \beta \delta \eta} W_{\mu \tau \chi, \lambda \sigma \zeta}\left(\frac{\left(X_{\lambda}^{n_{2}} X_{\sigma}^{n_{4}}\right)^{*} X_{\mu}^{n_{1}} X_{\gamma}^{n_{2}} X_{\tau}^{n_{3}} X_{\epsilon}^{n_{4}} X_{\chi}^{n_{5}} Y_{\delta}^{k_{6}} Y_{\eta}^{k_{7}} Y_{\zeta}^{k_{8}}}{\left(-\left(\varepsilon_{n_{2}}^{+}+\varepsilon_{n_{4}}^{+}-\varepsilon_{k_{6}}^{-}-\varepsilon_{k_{7}}^{-}\right)+i \eta\right)}\left(t_{k_{6} k_{7} k_{8}}^{n_{1} n_{3} n_{5}}\right)^{*}\right. \\
& \left.+\frac{\left(Y_{\gamma}^{k_{1}} Y_{\mu}^{k_{2}} Y_{\epsilon}^{k_{3}} Y_{\tau}^{k_{4}} Y_{\chi}^{k_{5}} X_{\delta}^{n_{6}} X_{\eta}^{n_{7}} X_{\zeta}^{n_{8}}\right)^{*} Y_{\lambda}^{k_{1}} Y_{\sigma}^{k_{3}}}{\varepsilon_{k_{1}}^{-}+\varepsilon_{k_{3}}^{-}-\varepsilon_{n_{6}}^{+}-\varepsilon_{n_{7}}^{+}+i \eta} t_{k_{2} k_{4} k_{5}}^{n_{n_{7}} n_{8}}\right) \text {. }
\end{aligned}
$$

Together with the last term of Eq. (C8), the third-order diagrams in Figs. 14e, 14i, 14j, 14k, 14l, 14m, and 14n are skeleton and therefore they would need to be included in a fully self-consistent ADC(3) formulation. Note, however, that Eq. C8 and the diagram 14 educe to a single contribution if the full $\widetilde{V}$, from Eq. (15), is used. Again, choosing an HF state as the unperturbed reference would force the diagrams of Figs. 14a, 14f, 14g and 14h to vanish.

\section{Dynamic self-energy}

When a self-consistent formulation is possible, some of the correlation effects beyond mean field are already included through the use of dressed reference propagators. However, for a reference state that is not dressed, additional nonskeleton diagrams contribute to the energydependent self-energy at third and higher orders. Hence, their contributions should be added to the $\mathrm{ADC}(n)$ equations.

Specifically, at the $\mathrm{ADC}(3)$ level, the one-particle irreducible and interaction-irreducible diagrams considered for the energy-dependent self-energy in Sec. III and Appendix A must be complemented with the four Feynman diagrams of Fig. 15. Diagrammatically, they are obtained by inserting the first-order $1 \mathrm{~B}$ effective interaction $\widetilde{U}^{(1)}$ of Fig. 9 into the second-order diagrams of Fig. 1. Since we are not considering four and higher orders here, only the $\widetilde{U}^{(1)}$ needs to be included for $\operatorname{ADC}(3)$.

In this case, the $\mathrm{ADC}(3)$ expansion of the self-energy matrices that appear in Eq. 28 is enriched with additional coupling corrections. These are already listed in
Eqs. A1 and (A2), respectively for the forward-in-time and backward-in-time cases, and they are repeated here for completeness:

$$
\begin{aligned}
& \mathbf{M}_{j \alpha}^{(\mathrm{II})}= \begin{cases}\mathbf{M}_{r \alpha}^{(\mathrm{IIr})}+\mathbf{M}_{r \alpha}^{(\mathrm{IIs})} & \text { if } j=r(2 p 1 h), \\
\mathbf{M}_{q \alpha}^{(\mathrm{IIt})}+\mathbf{M}_{q \alpha}^{(\mathrm{IIu})} & \text { if } j=q(3 p 2 h),\end{cases} \\
& \mathbf{N}_{\alpha k}^{(\mathrm{II})}= \begin{cases}\mathbf{N}_{\alpha s}^{(\mathrm{IIr})}+\mathbf{N}_{\alpha s}^{(\mathrm{IIs})} & \text { if } k=s(2 h 1 p), \\
\mathbf{N}_{\alpha u}^{(\mathrm{IIt})}+\mathbf{N}_{\alpha u}^{(\mathrm{IIu})} & \text { if } k=u(3 h 2 p) .\end{cases}
\end{aligned}
$$

Also for the interaction matrices, the nonskeleton expansion is enriched by additional $\mathbf{C}_{j j^{\prime}}$ and $\mathbf{D}_{k k^{\prime}}$ matrices. 


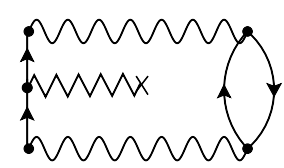

(r)

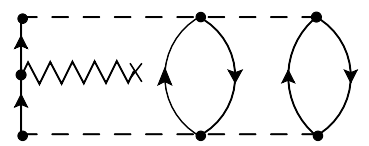

(t)

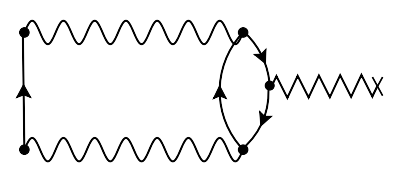

(s)

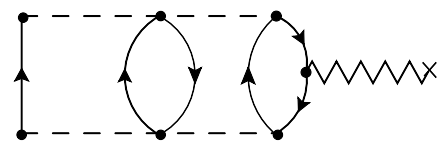

(u)
FIG. 15. Third-order nonskeleton diagrams of the dynamic self-energy, complementing the ones in Figs. 2 and 6 for the general $\mathrm{ADC}(3)$, not based on a self-consistent reference propagator. Zigzag lines represent the effective $1 \mathrm{~B}$ interaction of Eq. (C4).

These terms are included in Eqs. A3 A4 and are:

$$
\begin{aligned}
& \mathbf{C}_{j j^{\prime}}= \begin{cases}\mathbf{C}_{r r^{\prime}}^{\widetilde{U} p}+\mathbf{C}_{r r^{\prime}}^{\widetilde{U} h} & \text { if } j=r \text { and } j^{\prime}=r^{\prime}(2 p 1 h), \\
\mathbf{C}_{q q^{\prime}}^{\widetilde{U} p}+\mathbf{C}_{q q^{\prime}}^{\widetilde{U} h} & \text { if } j=q \text { and } j^{\prime}=q^{\prime}(3 p 2 h), \\
0 & \text { otherwise, }\end{cases} \\
& \mathbf{D}_{k k^{\prime}}= \begin{cases}\mathbf{D}_{s s^{\prime}}^{\widetilde{U} h}+\mathbf{D}_{s s^{\prime}}^{\widetilde{U} p} & \text { if } k=s \text { and } k^{\prime}=s^{\prime}(2 h 1 p), \\
\mathbf{D}_{u u^{\prime}}^{\widetilde{U} p}+\mathbf{D}_{u u^{\prime}}^{\widetilde{U} h} & \text { if } k=u \text { and } k^{\prime}=u^{\prime}(3 h 2 p), \\
0 & \text { otherwise. }\end{cases}
\end{aligned}
$$

\section{a. ADC(3) terms with $2 p 1 h$ and $2 h 1 p$ ISCs}

By following the same procedure used to find the expressions of the coupling matrices containing $2 \mathrm{NFs}$ and/or 3NFs, we can derive the analogous expressions of $\mathbf{M}_{j \alpha}$ and $\mathbf{N}_{\alpha k}$ containing one $\widetilde{U}$ insertion, and expressed in terms of the uncorrelated transition amplitudes of Eq. C1.

From the Goldstone-Feynman diagrams in Figs. 15r and $15 \mathrm{~s}$ we find,

$$
\mathbf{M}_{r \alpha}^{(\mathrm{IIr})} \equiv \frac{-1}{\sqrt{2}} \mathcal{A}_{12} \frac{X_{\gamma}^{n_{1}} \widetilde{U}_{\gamma \delta}^{(1)} Y_{\delta}^{k_{4}}}{\varepsilon_{k_{4}}^{-}-\varepsilon_{n_{1}}^{+}}\left(Y_{\mu}^{k_{4}}\right)^{*} X_{\nu}^{n_{2}} Y_{\lambda}^{k_{3}} \widetilde{V}_{\mu \nu, \alpha \lambda}
$$

and

$$
\mathbf{M}_{r \alpha}^{(\mathrm{IIs})} \equiv \frac{1}{\sqrt{2}} \frac{X_{\gamma}^{n_{4}} \widetilde{U}_{\gamma \delta}^{(1)} Y_{\delta}^{k_{3}}}{\varepsilon_{k_{3}}^{-}-\varepsilon_{n_{4}}^{+}} X_{\mu}^{n_{1}} X_{\nu}^{n_{2}}\left(X_{\lambda}^{n_{4}}\right)^{*} \widetilde{V}_{\mu \nu, \alpha \lambda}
$$

respectively.

The $2 p 1 h$ interaction matrices in Eq. C15 are

$$
\mathbf{C}_{r r^{\prime}}^{\widetilde{U} p}=\frac{1}{2} \mathcal{A}_{12} \mathcal{A}_{45} X_{\gamma}^{n_{1}} \widetilde{U}_{\gamma \delta}^{(1)}\left(X_{\delta}^{n_{4}}\right)^{*} \delta_{n_{2} n_{5}} \delta_{k_{3} k_{6}}
$$

and

$$
\mathbf{C}_{r r^{\prime}}^{\widetilde{U} h}=\frac{-1}{2} \mathcal{A}_{12} Y_{\delta}^{k_{3}} \widetilde{U}_{\gamma \delta}^{(1)}\left(Y_{\gamma}^{k_{6}}\right)^{*} \delta_{n_{1} n_{4}} \delta_{n_{2} n_{5}}
$$

In the backward-in-time Goldstone diagrams of Figs. $15 \mathrm{r}$ and $15 \mathrm{~s}$ one finds the coupling matrices,

$$
\mathbf{N}_{\alpha s}^{(\mathrm{IIr})} \equiv \frac{1}{\sqrt{2}} \widetilde{V}_{\alpha \lambda, \mu \nu} \mathcal{A}_{12} \frac{X_{\gamma}^{n_{4}} \widetilde{U}_{\gamma \delta}^{(1)} Y_{\delta}^{k_{1}}}{\varepsilon_{k_{1}}^{-}-\varepsilon_{n_{4}}^{+}}\left(X_{\mu}^{n_{4}}\right)^{*} Y_{\nu}^{k_{2}} X_{\lambda}^{n_{3}}
$$

and

$$
\mathbf{N}_{\alpha s}^{(\mathrm{IIs})} \equiv \frac{-1}{\sqrt{2}} \widetilde{V}_{\alpha \lambda, \mu \nu} \frac{X_{\gamma}^{n_{3}} \widetilde{U}_{\gamma \delta}^{(1)} Y_{\delta}^{k_{4}}}{\varepsilon_{k_{4}}^{-}-\varepsilon_{n_{3}}^{+}} Y_{\mu}^{k_{1}} Y_{\nu}^{k_{2}}\left(Y_{\lambda}^{k_{4}}\right)^{*},
$$

while the corresponding interaction matrices in Eq. C16 connecting $2 h 1 p$ ISCs, are

$$
\mathbf{D}_{s s^{\prime}}^{\widetilde{U} h}=\frac{1}{2} \mathcal{A}_{12} \mathcal{A}_{45}\left(Y_{\gamma}^{k_{1}}\right)^{*} \widetilde{U}_{\gamma \delta}^{(1)} Y_{\delta}^{k_{4}} \delta_{k_{2} k_{5}} \delta_{n_{3} n_{6}}
$$

and

$$
\mathbf{D}_{s s^{\prime}}^{\widetilde{U} p}=\frac{-1}{2} \mathcal{A}_{12}\left(X_{\delta}^{n_{3}}\right)^{*} \widetilde{U}_{\gamma \delta}^{(1)} X_{\gamma}^{n_{3}} \delta_{k_{1} k_{4}} \delta_{k_{2} k_{5}}
$$

Equations (C17)-C24 above are third-order terms composed by effective 1B interaction and effective 2NFs. In the next section we proceed by introducing the set of expressions with effective $1 \mathrm{~B}$ interaction and $3 \mathrm{NFs}$, which connect $3 p 2 h$ and $3 h 2 p$ ISCs. 


\section{b. $A D C(3)$ terms with $3 p 2 h$ and $3 h 2 p$ ISCs}

The Goldstone-Feynman diagrams of Figs. $15 \mathrm{t}$ and $15 \mathrm{u}$ involve $3 p 2 h$ and $3 h 2 p$ ISCs. They contain the coupling matrices that complete the expressions for $\mathbf{M}_{j \alpha}$ and $\mathbf{N}_{\alpha k}$, when the reference state adopted has not be calculated self-consistently.

The working equations for the forward-in-time coupling matrices are,

$$
\mathbf{M}_{q \alpha}^{(\mathrm{IIt})} \equiv \frac{-1}{\sqrt{12}} \mathcal{P}_{123} \frac{X_{\gamma}^{n_{1}} \widetilde{U}_{\gamma \delta}^{(1)} Y_{\delta}^{k_{6}}}{\varepsilon_{k_{6}}^{-}-\varepsilon_{n_{1}}^{+}}\left(Y_{\mu}^{k_{6}}\right)^{*} X_{\nu}^{n_{2}} X_{\rho}^{n_{3}} Y_{\lambda}^{k_{4}} Y_{\eta}^{k_{5}} W_{\mu \nu \rho, \alpha \lambda \eta}
$$

and

$$
\mathbf{M}_{q \alpha}^{(\mathrm{IIu})} \equiv \frac{1}{\sqrt{12}} \mathcal{A}_{45} \frac{X_{\gamma}^{n_{6}} \widetilde{U}_{\gamma \delta}^{(1)} Y_{\delta}^{k_{5}}}{\varepsilon_{k_{5}}^{-}-\varepsilon_{n_{6}}^{+}} X_{\mu}^{n_{1}} X_{\nu}^{n_{2}} X_{\rho}^{n_{3}} Y_{\lambda}^{k_{4}}\left(X_{\eta}^{n_{6}}\right)^{*} W_{\mu \nu \rho, \alpha \lambda \eta},
$$

while the interaction matrices in Eq. C15 connecting two $3 p 2 h$ ISCs are,

$$
\mathbf{C}_{q q^{\prime}}^{\widetilde{U} p}=\frac{1}{12} \mathcal{A}_{123} \mathcal{A}_{45} \mathcal{P}_{678} X_{\gamma}^{n_{1}} \widetilde{U}_{\gamma \delta}^{(1)}\left(X_{\delta}^{n_{6}}\right)^{*} \delta_{n_{2} n_{7}} \delta_{n_{3} n_{8}} \delta_{k_{4} k_{9}} \delta_{k_{5} k_{10}}
$$

and

$$
\mathbf{C}_{q q^{\prime}}^{\widetilde{U} h}=\frac{-1}{12} \mathcal{A}_{45} \mathcal{A}_{910} \mathcal{A}_{123} Y_{\delta}^{k_{5}} \widetilde{U}_{\gamma \delta}^{(1)}\left(Y_{\gamma}^{k_{10}}\right)^{*} \delta_{n_{1} n_{6}} \delta_{n_{2} n_{7}} \delta_{n_{3} n_{8}} \delta_{k_{4} k_{9}}
$$

Expressions for the backward-in-time coupling matrices, containing one effective 1B interaction and one interactionirreducible $3 \mathrm{NF}$ insertion, are

$$
\mathbf{N}_{\alpha u}^{(\mathrm{IIt})} \equiv \frac{1}{\sqrt{12}} W_{\alpha \lambda \eta, \mu \nu \rho} \mathcal{P}_{123} \frac{X_{\gamma}^{n_{6}} \widetilde{U}_{\gamma \delta}^{(1)} Y_{\delta}^{k_{1}}}{\varepsilon_{k_{1}}^{-}-\varepsilon_{n_{6}}^{+}}\left(X_{\mu}^{n_{6}}\right)^{*} Y_{\nu}^{k_{2}} Y_{\rho}^{k_{3}} X_{\lambda}^{n_{4}} X_{\eta}^{n_{5}}
$$

and

$$
\mathbf{N}_{\alpha u}^{(\mathrm{IIu})} \equiv \frac{-1}{\sqrt{12}} W_{\alpha \lambda \eta, \mu \nu \rho} \mathcal{A}_{45} \frac{X_{\gamma}^{n_{5}} \widetilde{U}_{\gamma \delta}^{(1)} Y_{\delta}^{k_{6}}}{\varepsilon_{k_{6}}^{-}-\varepsilon_{n_{5}}^{+}} Y_{\mu}^{k_{1}} Y_{\nu}^{k_{2}} Y_{\rho}^{k_{3}} X_{\lambda}^{n_{4}}\left(Y_{\eta}^{k_{6}}\right)^{*}
$$

while the interaction matrices in Eq. C16 connecting two $3 h 2 p$ ISCs are

$$
\mathbf{D}_{u u^{\prime}}^{\widetilde{U} p}=-\frac{1}{12} \mathcal{A}_{45} \mathcal{A}_{910} \mathcal{A}_{123}\left(X_{\delta}^{n_{5}}\right)^{*} \widetilde{U}_{\gamma \delta}^{(1)} X_{\gamma}^{n_{10}} \delta_{k_{1} k_{6}} \delta_{k_{2} k_{7}} \delta_{k_{3} k_{8}} \delta_{n_{4} n_{9}}
$$

and

$$
\mathbf{D}_{u u^{\prime}}^{\widetilde{U} h}=\frac{1}{12} \mathcal{A}_{123} \mathcal{A}_{45} \mathcal{P}_{678}\left(Y_{\gamma}^{k_{1}}\right)^{*} \widetilde{U}_{\gamma \delta}^{(1)} Y_{\delta}^{k_{6}} \delta_{k_{2} k_{7}} \delta_{k_{3} k_{8}} \delta_{n_{4} n_{9}} \delta_{n_{5} n_{10}}
$$

[1] H.-W. Hammer, A. Nogga, and A. Schwenk, Rev. Mod. Phys. 85, 197 (2013)

[2] C. Barbieri and A. Carbone, in An Advanced Course in Computational Nuclear Physics: Bridging the Scales from Quarks to Neutron Stars, edited by M. HjorthJensen, M.P. Lombardo, and U. van Kolck, Lecture Notes in Physics Vol. 936 (Springer, 2017) pp. 571-644.

[3] A. Cipollone, C. Barbieri, and P. Navrátil, Phys. Rev. Lett. 111, 062501 (2013).

[4] G. Hagen, T. Papenbrock, M. Hjorth-Jensen, and D. J.
Dean, Reports on Progress in Physics 77, 096302 (2014)

[5] S. Binder, P. Piecuch, A. Calci, J. Langhammer, P. Navrátil, and R. Roth, Phys. Rev. C 88, 054319 (2013)

[6] K. Tsukiyama, S. K. Bogner, and A. Schwenk, Phys. Rev. Lett. 106, 222502 (2011)

[7] H. Hergert, S. Bogner, T. Morris, A. Schwenk, and K. Tsukiyama, Physics Reports 621, 165 (2016), memorial Volume in Honor of Gerald E. Brown.

[8] E. Epelbaum, H.-W. Hammer, and U.-G. Meißner, Rev. 
Mod. Phys. 81, 1773 (2009)

[9] R. Machleidt and D. Entem, Physics Reports 503, 1 (2011)

[10] K. Hebeler, J. Holt, J. Menéndez, and A. Schwenk, Annual Review of Nuclear and Particle Science 65, 457 (2015), https://doi.org/10.1146/annurev-nucl102313-025446

[11] C. Barbieri, Journal of Physics: Conference Series 529, 012005 (2014)

[12] V. Lapoux, V. Somà, C. Barbieri, H. Hergert, J. D. Holt, and S. R. Stroberg, Phys. Rev. Lett. 117, 052501 (2016)

[13] A. Calci and R. Roth, Phys. Rev. C 94, 014322 (2016)

[14] C. Barbieri and W. H. Dickhoff, Phys. Rev. C 68, 014311 (2003)

[15] W. Dickhoff and C. Barbieri, Progress in Particle and Nuclear Physics 52, 377 (2004)

[16] G. Onida, L. Reining, and A. Rubio, Rev. Mod. Phys. 74, 601 (2002).

[17] F. Aryasetiawan and O. Gunnarsson, Reports on Progress in Physics 61, 237 (1998)

[18] W. von Niessen, J. Schirmer, and L. Cederbaum, Computer Physics Reports 1, 57 (1984)

[19] D. Danovich, Wiley Interdisciplinary Reviews: Computational Molecular Science 1, 377 (2011)

[20] J. Schirmer, Phys. Rev. A 26, 2395 (1982)

[21] J. Schirmer, L. S. Cederbaum, and O. Walter, Phys. Rev. A 28, 1237 (1983)

[22] A. Carbone, A. Cipollone, C. Barbieri, A. Rios, and A. Polls, Phys. Rev. C 88, 054326 (2013).

[23] V. Somà, A. Cipollone, C. Barbieri, P. Navrátil, and T. Duguet, Phys. Rev. C 89, 061301 (2014)

[24] A. Cipollone, C. Barbieri, and P. Navrátil, Phys. Rev. C 92, 014306 (2015)

[25] A. Carbone, A. Polls, and A. Rios, Phys. Rev. C 88, $044302(2013)$

[26] A. Idini and C. Barbieri, Acta Phys. Pol. B 88, 273 (2017)
[27] W. Dickhoff and D. Van Neck, Many-body Theory Exposed!: Propagator Description of Quantum Mechanics in Many-body Systems, EBSCO ebook academic collection (World Scientific, 2005).

[28] A. L. Fetter and J. D. Walecka, Quantum Theory of Many-Particle Systems (McGraw-Hill, Boston, 1971).

[29] S. J. Waldecker, C. Barbieri, and W. H. Dickhoff, Phys. Rev. C 84, 034616 (2011).

[30] P. Grangé, A. Lejeune, M. Martzolff, and J.-F. Mathiot, Phys. Rev. C 40, 1040 (1989)

[31] V. Somà and P. Bożek, Phys. Rev. C 78, 054003 (2008)

[32] K. Hebeler and A. Schwenk, Phys. Rev. C 82, 014314 (2010)

[33] K. Hebeler, S. K. Bogner, R. J. Furnstahl, A. Nogga, and A. Schwenk, Phys. Rev. C 83, 031301 (2011).

[34] V. Somà, T. Duguet, and C. Barbieri, Phys. Rev. C 84, 064317 (2011)

[35] V. Somà, C. Barbieri, and T. Duguet, Phys. Rev. C 89, 024323 (2014)

[36] I. Shavitt and R. Bartlett, Many-Body Methods in Chemistry and Physics: MBPT and Coupled-Cluster Theory Cambridge Molecular Science (Cambridge University Press, 2009).

[37] F. Raimondi and C. Barbieri, in preparation.

[38] F. Raimondi and C. Barbieri, Proceeding of the International Conference 'Nuclear Theory in the Supercomputing Era - 2016 (NTSE-2016)' edited by A.M. Shirokov and A.I. Mazur, Pacific National University, Khabarovsk, Russia (2018).

[39] O. Walter and J. Schirmer, J. Phys. B: At. Mol. Phys. 14, 3805 (1981).

[40] C. Barbieri, Phys. Lett. B 643, 268 (2006).

[41] C. Barbieri, D. Van Neck, and W. H. Dickhoff, Phys. Rev. A 76, 052503 (2007).

[42] S. P. Pandya, Phys. Rev. 103, 956 (1956) 